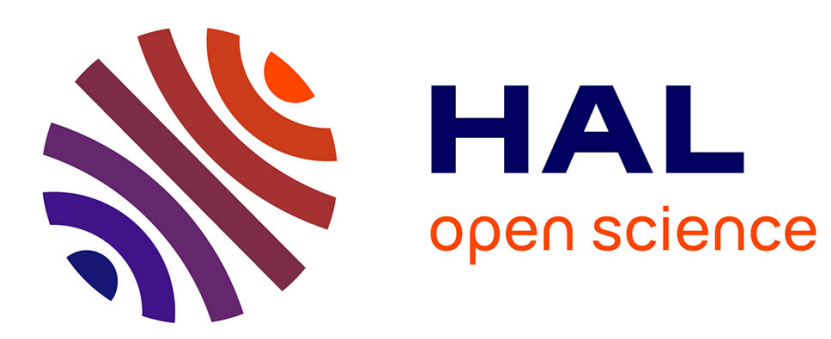

\title{
Nonsmooth simulation of dense granular flows with pressure-dependent yield stress
}

\author{
Gilles Daviet, Florence Bertails-Descoubes
}

\section{To cite this version:}

Gilles Daviet, Florence Bertails-Descoubes. Nonsmooth simulation of dense granular flows with pressure-dependent yield stress. Journal of Non-Newtonian Fluid Mechanics, 2016, 234, pp.15-35. 10.1016/j.jnnfm.2016.04.006 . hal-01236488v2

\section{HAL Id: hal-01236488 \\ https://hal.inria.fr/hal-01236488v2}

Submitted on 13 Apr 2016

HAL is a multi-disciplinary open access archive for the deposit and dissemination of scientific research documents, whether they are published or not. The documents may come from teaching and research institutions in France or abroad, or from public or private research centers.
L'archive ouverte pluridisciplinaire HAL, est destinée au dépôt et à la diffusion de documents scientifiques de niveau recherche, publiés ou non, émanant des établissements d'enseignement et de recherche français ou étrangers, des laboratoires publics ou privés.

\section{(ㅇ)(1) $\$$}

Distributed under a Creative Commons Attribution - NonCommercial - NoDerivatives $\mid 4.0$ 


\title{
Nonsmooth simulation of dense granular flows with pressure-dependent yield stress
}

Gilles Daviet

\author{
Florence Bertails-Descoubes*
}

April 13, 2016

\begin{abstract}
Understanding the flow of granular materials is of utmost importance for numerous industrial applications including the manufacturing, storing and transportation of grain assemblies (such as cement, pills, or corn), as well as for natural risk assessing considerations. Discrete Element Modeling (DEM) methods, which explicitly represent graingrain interactions, allow for highly-tunable and precise simulations, but they suffer from a prohibitive computational cost when attempting to reproduce large scale scenarios. Continuum models have been recently investigated to overcome such scalability issues, but their numerical simulation still poses many challenges. In this work we propose a novel numerical framework for the continuous simulation of dilatable materials with pressure-dependent (Coulomb) yield stress. Relying upon convex optimization tools, we show that such a macroscopic, nonsmooth rheology can be interpreted as the exact analogous of the solid frictional contact problem at the heart of DEM methods, extended to the tensorial space. Combined with a carefully chosen finite-element discretization, this new framework allows us to avoid regularizing the continuum rheology while benefiting from the efficiency of nonsmooth optimization solvers, mainly leveraged by DEM methods so far. Our numerical results successfully compare to analytic solutions on model problems, and we retrieve qualitative flow features commonly observed in reported experiments of the literature.
\end{abstract}

\section{Introduction}

\subsection{Motivation}

Granular media are common both naturally and in industrial processes, and understanding their flow is crucial to several applications, such as studying the evolution of the pressure in a silo discharge or predicting the run-out of avalanches. Consequently, the past decades have yielded extensive bodies of research dedicated to the modeling of such materials. However, to this day their numerical simulation remains a challenging problem.

In this work we focus on a continuum model for granular-like materials satisfying two key properties. First, we assume that the material cannot be compacted, while it is allowed to dilate as desired, i.e., the divergence of the velocity field is non-negative everywhere, but not necessarily equal to zero. Second, we consider that the material behaves as a viscoplastic fluid whose yield stress depends linearly on the local pressure. Intuitively, this comes from the assumption that grain-grain interactions mostly involve rigid-body contacts with Coulomb friction.

*INRIA Grenoble Rhône-Alpes and Laboratoire Jean Kuntzman, 655 avenue de l'Europe, 38334 Saint- Ismier, France. \{gilles.daviet,florence\}.descoubes@inria.fr

\subsection{Related work}

\subsubsection{Discrete Elements Modeling}

A natural way to model granular materials is to proceed at the grain level.

Stemming from the seminal work of Jean-Jacques Moreau, nonsmooth methods for simulating frictional contacts between discrete bodies have been the subject of extensive research in the last three decades. Alart and Curnier [Alart and Curnier, 1991] proposed one of the first formulation of Coulomb friction that is well-suited for nonsmooth optimization methods. Using an operator-splitting scheme, Jean and colleagues [Jourdan et al., 1998, Jean, 1999] managed to make it scale to very large number of contacts. Their work became known as the Nonsmooth Contact Dynamics (NSCD) method. As it will be of great relevance in the technical content of this paper, we also point out the change of variable introduced by De Saxcé and Feng [De Saxcé and Feng, 1998] who reformulated the discrete-time contact problem as a nonlinear) Second-Order Cone complementarity problem. Cadoux [Cadoux, 2009] used this point of view to formulate a criterion for the existence of solutions, as well as an algorithm for finding one solution, relying on a series of Second-Order Cone Quadratic Programs.

Those grain-level methods are still the subject of extensive work in the context of granular materials, in regard 
of both their computational efficiency [Alart et al., 2012, Kleinert et al., 2014] and their ability to describe specific materials [Lim et al., 2014].

However, since they have to take into account every contact between grains, the cost of DEM methods increases tremendously when considering scenarios where the particles are very small w.r.t. the computational domain. This motivates the need for a continuum description of the material. Some authors have also developed hybrid methods which attempt to retain the precision of DEM while tackling larger computational domains [Bardenhagen et al., 2000, Wellmann and Wriggers, 2012]. In this case, an efficient way to solve the dynamics of the continuous part of the model is still required.

\subsubsection{Continuous modeling of granular materials}

In the last decade, the introduction of the $\mu(I)$ rheology [Jop et al., 2006, GDR MiDi, 2004] contributed to the growth of a large body of research on continuous models for dry granular materials. In particular, the $\mu(I)$ rheology was validated against Discrete Element Modeling simulations on complex scenarios such as the collapse of a granular column [Lagrée et al., 2011] or the discharge of silos [Kamrin, 2010, Staron et al., 2014].

In a very recent work, Ionescu et al. [Ionescu et al., 2015] were able to reproduce experimental collapses of granular columns using either the full $\mu(I)$ rheology, or a constant friction coefficient $\mu$ and a constant Newtonian viscosity. Here we shall focus on the latter case of a constant $\mu$, though we show in Section 6.2.2 that our method may accommodate more complex rheologies with little modification.

Several works have focused on the simulation of such a model, most of them considering the flow to be incompressible [Lagrée et al., 2011, Chambon et al., 2011, Staron et al., 2014, Ionescu et al., 2015, Chauchat and Médale, 2014]. However, this choice may prove unfortunate in certain configurations, such as the wake of an obstacle, where special care has to be taken to ensure that the rheology remains well-defined [Chauchat and Médale, 2014]. In contrast, Dunatunga and Kamrin [Dunatunga and Kamrin, 2015] recently used an explicit Material Point Method to model compressible granular flows. In a similar manner, our method does not preclude the expansion of the flow. However, we consider a purely rigid solid regime instead of an elastic one, and focus our analysis on the dense limit. Compared to prior art, the main novelty of our approach is to propose to solve the rheology constraints in an implicit manner, by leveraging tools from nonsmooth optimization. This allows us to capture directly the steady-state of non-inertial flows.

\subsubsection{Numerical simulation of yield-stress flows}

Yield-stress fluids, especially those exhibiting Bingham or Herschel-Bulkley rheologies, have been widely investigated experimentally and numerically, due to their numerous industrial applications. Due to the nondifferentiability of their rheology, simulating yield-stress fluids using an explicit scheme requires the use of very small time-steps; on the other hand, devising implicit methods pose theoretical difficulties. Such implicit approaches are usually instances of either:

- Regularizing methods, which employ diverse numerical artifacts to smooth out the singularities of the rheology (see, e.g., [Frigaard and Nouar, 2005] for a review). The main benefit of a regularizing strategy is the use of classical numerical schemes dedicated to differential equations. However such methods may lead to stiff equations, making them unable to capture fully rigid zones properly [Saramito and Roquet, 2001];

- Augmented Lagrangian methods, based on the framework of [Fortin and Glowinski, 1982]. Their main drawback is the slow convergence rate often observed in practice.

Very recently, Bleyer et al. [Bleyer et al., 2015] proposed a method for Herschel-Bulkley fluids based on the nonsmooth convex optimization theory, which they claim benefits from much faster convergence properties than Augmented Lagrangian methods. In a similar spirit, our approach builds a nonsmooth model for the class of dilatable and pressure-dependent yield stress flows (such as granular materials), and leverages the efficiency of optimization-based solvers for making the numerical solving tractable.

\subsection{Contributions}

More specifically, our contributions include:

- A viscoplastic rheology for dense granular flows with a Drucker-Prager yielding criterion and an unilateral incompressibility constraint;

- A finite-element formulation that exhibits at the discrete level a strong similarity with discrete inelastic contact mechanics;

- Algorithms for solving these equations based on the framework of [Cadoux, 2009];

- A numerical validation of the overall method, including the retrieval of qualitative experimental features.

\subsection{Organization}

In Section 2 we introduce the main notations used throughout the paper. Section 3 presents our granular rheology and shows how it can be conveniently reformulated as the exact analogous of the solid frictional contact law. In Section 4, a finite element approximation of the flow equations is proposed for the creeping case, yielding a discrete problem which turns out to be very similar to the one encountered in DEM. Nonsmooth optimization based algorithms developed in the solid frictional contact community are then leveraged to solve our problem 
efficiently. Those results are extended to inertial flows in Section 5. Finally, we present in Section 6 our main simulation results as well as some validation against experimental and numerical studies, before discussing the merits and limitations of our approach in Section 7.

\section{Notation}

Let $d$ be the dimension of the space (in practice $d=2$ or $d=3)$. Let $S_{d}$ be the space of symmetric $d \times d$ tensors. Let $\boldsymbol{\sigma} \in S_{d}$ and $\boldsymbol{\tau} \in S_{d}$, and $\boldsymbol{\sigma}: \boldsymbol{\tau}=\sum_{i, j} \boldsymbol{\sigma}_{i, j} \boldsymbol{\tau}_{i, j} \in \mathbb{R}$ be the twice-contracted product between $\boldsymbol{\sigma}$ and $\boldsymbol{\tau}$. In the following we shall use the scalar product $\langle\boldsymbol{\sigma}, \boldsymbol{\tau}\rangle:=\frac{\boldsymbol{\sigma}: \boldsymbol{\tau}}{2}$ on $S_{d}$ and its associated norm $|\boldsymbol{\sigma}|:=\sqrt{\langle\boldsymbol{\sigma}, \boldsymbol{\sigma}\rangle}$.

The trace of $\boldsymbol{\sigma}$ is a scalar denoted as $\operatorname{Tr}(\boldsymbol{\sigma})$. The traceless (or deviatoric) part of $\boldsymbol{\sigma}$ is a symmetric $d \times d$ tensor defined as $\operatorname{Dev}(\boldsymbol{\sigma}):=\boldsymbol{\sigma}-\frac{1}{d} \mathbf{I} \operatorname{Tr}(\boldsymbol{\sigma})$, such that $\operatorname{Tr}(\operatorname{Dev}(\boldsymbol{\sigma}))=0$.

Let $\mathbf{u}$ be the velocity field of the fluid, that is a function from $\mathbb{R}^{d} \times \mathbb{R}$ to $\mathbb{R}^{d}$ such that $\mathbf{u}(\mathbf{x}, t)$ is the macroscopic velocity of the fluid that is passing through $\mathbf{x}$ at time $t$. The velocity gradient of the fluid is a $d \times d$ tensor denoted $\nabla \mathbf{u}$, which contains on its $j^{\text {th }}$ row the gradient vector of the $j^{\text {th }}$ component of $\mathbf{u}$. The symmetric part of the velocity gradient, which will be our main variable of interest throughout the paper, is a symmetric $d \times d$ tensor defined as $\mathrm{D}(\mathbf{u}):=\frac{1}{2}\left(\nabla \mathbf{u}+\nabla \mathbf{u}^{T}\right)$. We have $\operatorname{Tr}(\mathrm{D}(\mathbf{u}))=$ $\nabla \cdot \mathbf{u}$ and $\operatorname{Dev}(\mathrm{D}(\mathbf{u}))=\mathrm{D}(\mathbf{u})-\frac{1}{d} \mathbf{I} \nabla \cdot \mathbf{u}$.

Finally, we introduce the symmetric tensor $\mathrm{D}^{b}(\mathbf{u}):=$ $\mathrm{D}(\mathbf{u})+\frac{\mathrm{b}-1}{d} \mathbf{I} \nabla \cdot \mathbf{u}$, where $b$ is a non-negative scalar. We have $\mathrm{D}^{0}(\mathbf{u})=\operatorname{Dev}(\mathrm{D}(\mathbf{u}))$ and $\mathrm{D}^{1}(\mathbf{u})=\mathrm{D}(\mathbf{u})$.

\section{$3 \quad$ Rheology $\mathcal{D P}\left(\mu, \sigma_{0}\right)$}

\subsection{Definition}

\subsubsection{Pressure-dependent yield-stress fluid}

We consider a rheology combining a constant Newtonian viscosity $\eta$ with a yield-stress $\kappa_{\mu, \boldsymbol{\sigma}_{0}}(p)$ that linearly depends on the pressure $p$,

$$
\kappa_{\mu, \sigma_{0}}(p)=\sigma_{0}+\mu p,
$$

similarly as in [Ionescu et al., 2015].

The total stress tensor $\boldsymbol{\sigma}_{t o t}$ is defined as ${ }^{1}$

$$
\boldsymbol{\sigma}_{t o t}:=2 \eta \mathrm{D}^{b}(\mathbf{u})+\boldsymbol{\tau}-p \mathbf{I},
$$

where $\tau$ is a traceless symmetric tensor satisfying

$$
\begin{cases}\boldsymbol{\tau}=\kappa_{\mu, \boldsymbol{\sigma}_{0}}\left(\sqrt{\frac{d}{2}} p\right) \frac{\mathrm{D}^{0}(\mathbf{u})}{\left|\mathrm{D}^{0}(\mathbf{u})\right|} & \text { if } \mathrm{D}^{0}(\mathbf{u}) \neq \mathbf{0} \\ |\boldsymbol{\tau}| \leq \kappa_{\mu, \boldsymbol{\sigma}_{0}}\left(\sqrt{\frac{d}{2}} p\right) & \text { if } \mathrm{D}^{0}(\mathbf{u})=\mathbf{0},(1 \mathrm{~b})\end{cases}
$$

\footnotetext{
${ }^{1}$ Using the more general $\mathrm{D}^{b}(\mathbf{u})$ rather than the usual $\mathrm{D}^{0}(\mathbf{u})$ for the Newtonian viscosity allows us to model a possibly non-zero bulk viscosity.
}

We chose to incorporate the factor $\sqrt{\frac{d}{2}}$ in the above definition in order to lighten the notations in the following sections. Note that in the $2 \mathrm{D}$ case, $\mu$ coincides with the usual Mohr-Coulomb definition of the friction coefficient: $\mu:=\sin \varphi$, with $\varphi$ the angle of friction.

Remark 1. We used $|\boldsymbol{\tau}|$ in (1b) instead of the usual $\left|\operatorname{Dev}\left(\boldsymbol{\sigma}_{t o t}\right)\right|$. But since $\operatorname{Dev}\left(\boldsymbol{\sigma}_{t o t}\right)=2 \eta b \mathrm{D}^{0}(\mathbf{u})+\boldsymbol{\tau}$, in the non-flowing case (1b) where $\mathrm{D}^{0}(\mathbf{u})=0$, we have $\left|\operatorname{Dev}\left(\boldsymbol{\sigma}_{t o t}\right)\right|=|\boldsymbol{\tau}|$.

\subsubsection{Dilatability}

Most continuum-based models for granulars consider the fluid to be perfectly incompressible. In contrast, we want to take into account the typically asymmetric yielding behavior of granulars by allowing the fluid to expand as much as desired, while strictly preventing compaction.

Dense flow hypothesis Dunatunga et al. [Dunatunga and Kamrin, 2015] model such an anisotropic behavior by considering two regimes; an elastoplastic regime when the density of the flow is above a critical value, and a free-flowing regime otherwise. They keep track of this density field over time thanks to a set of Lagrangian particles. However, here we will consider simpler scenarios, and make the assumption that the flow is always dense - i.e., everywhere at its maximal density. As such, we consider the density to be constant over the domain and over time.

The main advantage of this hypothesis is that it avoids having to couple the momentum and mass conservation equations, side-stepping several technical difficulties and allowing for a much more concise exposition of our implicit numerical method, which is our main contribution. Moreover, by carefully choosing the temporal and spatial discretization of a constraint enforcing a maximum density, we could adapt the framework presented in this article to accommodate arbitrary flows. We keep such investigations for future work.

Note however that the constant density assumption means that mass conservation will not hold inside the dilating zones. Fortunately, this inconsistency will have little effect on the predicted flow inside the dense regions. As validated in our Results section, our simplified model still remains applicable to a variety of relevant scenarios.

Complementarity condition With this dense flow hypothesis, the unilateral compressibility constraint can be expressed simply as $\nabla \cdot \mathbf{u} \geq 0$. We set the pressure $p$ to enforce this inequality, i.e.,

$$
\begin{cases}p \geq 0 & \text { if } \nabla \cdot \mathbf{u}=0 \\ p=0 & \text { if } \nabla \cdot \mathbf{u}>0\end{cases}
$$

or, using an equivalent complementarity notation,

$$
0 \leq p \perp \nabla \cdot \mathbf{u} \geq 0,
$$

where the notation $\alpha \perp \beta$ with $\alpha \in \mathbb{R}$ and $\beta \in \mathbb{R}$ means that at least one of the two scalars should vanish. If they 
are furthermore requested to be nonnegative, which is our case here, it means that $\alpha$ and $\beta$ cannot both be strictly positive at the same time.

In our results section, we shall see that relaxing the common incompressibility assumption $\nabla \cdot \mathbf{u}=0$ prevents the arising of an ill-defined rheology in some typical scenarios such as the flow in the wake of an obstacle [Chauchat and Médale, 2014]. Moreover our complementarity constraint (2) naturally fits in with our numerical framework, without adding any computational cumbersomeness.

\subsubsection{Cases covered by our choice of rheology}

Much like in [Ionescu et al., 2015], our set of parameters allows us to explore an interesting range of constitutive laws. When $\mu=0$, we retrieve the viscoplastic Bingham rheology, while when taking $\eta=0$ and $\sigma_{0}=0$, we get a purely Coulomb plastic flow - or, in other terms, the $\mu(I)$ rheology with a constant inertial number $I$.

The numerical method for the quasistatic simulation presented in Section 4 will require a non-zero $\eta$ to obtain a well-posed system. However, this constraint will be alleviated in Section 5 when using temporal schemes. In our numerical experiments, we were also able to simulate the complete $\mu(I)$ rheology by explicitly computing the inertial number $I$ at each time step.

Note also that the $\sigma_{0}$ coefficient should not be assimilated to the usual cohesion term. Indeed, in our case the additional stress due to this coefficient will always be tangential, and may be non-zero when $\nabla \cdot \mathbf{u}>0$. In this regard, $\sigma_{0}$ should rather be used to model effects related to the geometry of grains. If we were to model proper cohesion $c$, we could do so by defining $\kappa(p):=\mu(p+c)$, and the pressure unilateral constraint as $0 \leq p+c \perp \nabla \cdot \mathbf{u} \geq 0$. However in the framework presented below, thanks to a change of variable this would be strictly equivalent to adding a positive confining pressure to the right-hand side of the conservation of momentum equation. In the following, we shall thus free ourselves from modeling such a cohesion effect explicitly.

\subsubsection{Solution set $\mathcal{D} \mathcal{P}\left(\mu, \sigma_{0}\right)$}

Let us introduce the symmetric tensor $\boldsymbol{\lambda}:=2 \eta \mathrm{D}^{b}(\mathbf{u})-$ $\boldsymbol{\sigma}_{\text {tot }}=p \mathbf{I}-\boldsymbol{\tau}$. Using the identities $p=\frac{1}{d} \operatorname{Tr}(\boldsymbol{\lambda})$ and $\boldsymbol{\tau}=$ - Dev $\boldsymbol{\lambda}$, and the definition of $\mathrm{D}(\mathbf{u})$, our Drucker-Prager rheology $(1-2)$ can be directly rewritten as

$$
\begin{aligned}
& \int \operatorname{Dev}(\boldsymbol{\lambda})=-\kappa_{\mu, \boldsymbol{\sigma}_{0}}\left(\frac{\operatorname{Tr}(\boldsymbol{\lambda})}{\sqrt{2 d}}\right) \frac{\operatorname{Dev}(\mathrm{D}(\mathbf{u}))}{|\operatorname{Dev}(\mathrm{D}(\mathbf{u}))|} \\
& \text { if } \operatorname{Dev}(\mathrm{D}(\mathbf{u})) \neq \mathbf{0} \text {, } \\
& |\operatorname{Dev}(\boldsymbol{\lambda})| \leq \kappa_{\mu, \boldsymbol{\sigma}_{0}}\left(\frac{\operatorname{Tr}(\boldsymbol{\lambda})}{\sqrt{2 d}}\right) \text { if } \operatorname{Dev}(\operatorname{D}(\mathbf{u}))=\mathbf{0}, \\
& 0 \leq \frac{1}{d} \operatorname{Tr}(\boldsymbol{\lambda}) \perp \operatorname{Tr}(\mathrm{D}(\mathbf{u})) \geq 0 .
\end{aligned}
$$

In the remainder of this paper, we shall denote by $\mathcal{D P}\left(\mu, \sigma_{0}\right) \subset S_{d}^{2}$ the set of tensors $(\boldsymbol{\lambda} ; \mathrm{D}(\mathbf{u})) \in S_{d} \times S_{d}$ satisfying (3).

\subsection{Analogy with solid frictional contact}

\subsubsection{Solid Signorini-Coulomb law}

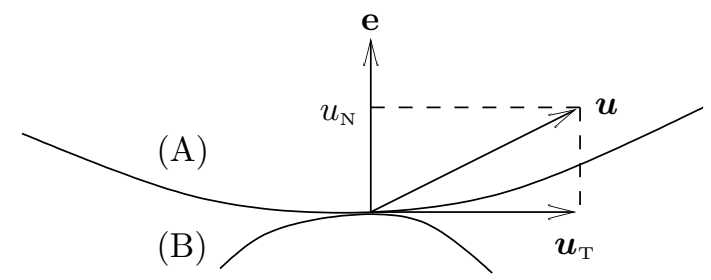

Figure 1: Local contact basis, with normal and tangent subspaces.

We briefly recall the Signorini-Coulomb friction law in the case of two solid objects (A) and (B) with a single contact point, as illustrated in Figure 1 . Let $\boldsymbol{u} \in \mathbb{R}^{d}$ be the relative velocity between the two colliding objects, $\boldsymbol{u}:=\boldsymbol{u}_{A}-\boldsymbol{u}_{B}$, and $\boldsymbol{r} \in \mathbb{R}^{d}$ be the reaction force applied by object (B) onto object (A). We suppose that the surface of contact is sufficiently smooth so that the normal $\mathbf{e} \in \mathbb{R}^{d}$ at the contact point can be uniquely defined.

Disjunctive formulation The Signorini-Coulomb law for contact with dry friction states that the couple $(\boldsymbol{r}, \boldsymbol{u})$ has to satisfy at least one of the following cases,

$$
\left\{\begin{array}{cc}
\boldsymbol{r}=0 \text { and } u_{\mathrm{N}} \geq 0 & \text { (Take-off) } \\
\left\|\boldsymbol{r}_{\mathrm{T}}\right\| \leq \mu r_{\mathrm{N}} \text { and } \boldsymbol{u}=0 & \text { (Sticking) } \\
\left\|\boldsymbol{r}_{\mathrm{T}}\right\|=\mu r_{\mathrm{N}} \text { and }\left\{\begin{array}{l}
u_{\mathrm{N}}=0 \\
\exists \alpha>0, \boldsymbol{u}_{\mathrm{T}}=-\alpha \boldsymbol{r}_{\mathrm{T}}
\end{array}\right. & \text { (Sliding) }
\end{array}\right.
$$

where $x_{\mathrm{N}} \in \mathbb{R}$ and $\boldsymbol{x}_{\mathrm{T}} \in \mathbb{R}^{d-1}$ are the normal and tangential components of vector $\boldsymbol{x} \in \mathbb{R}^{d}$, respectively, $\mu$ is the coefficient of friction at contact point, and $\|\cdot\|$ is the Euclidean norm on $\mathbb{R}^{d}$. Let us denote by $\mathcal{C}(\mu) \subset \mathbb{R}^{d} \times \mathbb{R}^{d}$ the set of $(\boldsymbol{r}, \boldsymbol{u})$ satisfying (4).

Functional formulations Though classical, the disjunctive formulation (4) is not always the most convenient to work with in a numerical setup. Typically, one may prefer to express the Signorini-Coulomb law as a root-finding problem, i.e., in the form

$$
(\boldsymbol{r}, \boldsymbol{u}) \in \mathcal{C}(\mu) \Longleftrightarrow f(\boldsymbol{r}, \boldsymbol{u})=0,
$$

where $f$ is a nonsmooth function from $\mathbb{R}^{d} \times \mathbb{R}^{d}$ to $\mathbb{R}^{d}$.

One classical example of such a nonsmooth function was notably provided by Alart and Curnier [Alart and Curnier, 1991], and defined as

$$
\begin{aligned}
f_{\mathrm{AC}}: \mathbb{R}^{d} \times \mathbb{R}^{d} & \longrightarrow \\
(\boldsymbol{r}, \boldsymbol{u}) & \longmapsto\left(\begin{array}{l}
\Pi_{\mathbb{R}_{+}}\left(r_{\mathrm{N}}-\xi_{\mathrm{N}} u_{\mathrm{N}}\right)-r_{\mathrm{N}} \\
\Pi_{\mathcal{B}^{d-1}\left(\mu r_{\mathrm{N}}\right)}\left(\boldsymbol{r}_{\mathrm{T}}-\xi_{\mathrm{T}} \boldsymbol{u}_{\mathrm{T}}\right)-\boldsymbol{r}_{\mathrm{T}}
\end{array}\right),
\end{aligned}
$$


where $\Pi_{\mathcal{C}}$ is the orthogonal projection operator on the convex space $\mathcal{C}, \xi_{\mathrm{T}}$ and $\xi_{\mathrm{N}}$ are positive real numbers, and $\mathcal{B}^{d-1}(a) \subset \mathbb{R}^{d-1}$ is the ball of radius $a \geq 0$ centered at the origin.

Another well-known function satisfying may be derived based upon De Saxcé and Feng [De Saxcé and Feng, 1998]'s work. Indeed, thanks to their proposed change of variable, $\hat{\boldsymbol{u}}=\boldsymbol{u}+\mu\left\|\boldsymbol{u}_{\mathrm{T}}\right\| \mathbf{e}$, the Coulomb law may be written compactly as

$$
\mathcal{K}_{\frac{1}{\mu}}^{d} \ni \hat{\boldsymbol{u}} \perp \boldsymbol{r} \in \mathcal{K}_{\mu}^{d},
$$

where the complementarity notation refers to the standard orthogonality in $\mathbb{R}^{d}$, and where $\mathcal{K}_{\mu}^{d} \subset \mathbb{R}^{d}$ is the second-order cone (SOC) of aperture $\mu$ (see Figure 2, left), generally defined on $\mathbb{R}^{n}(n \geq 2)$ as

$$
\mathcal{K}_{\mu}^{n}:=\left\{\left(x_{\mathrm{N}}, \boldsymbol{x}_{\mathrm{T}}\right) \in \mathbb{R} \times \mathbb{R}^{n-1},\left\|\boldsymbol{x}_{\mathrm{T}}\right\| \leq \mu x_{\mathrm{N}}\right\} .
$$
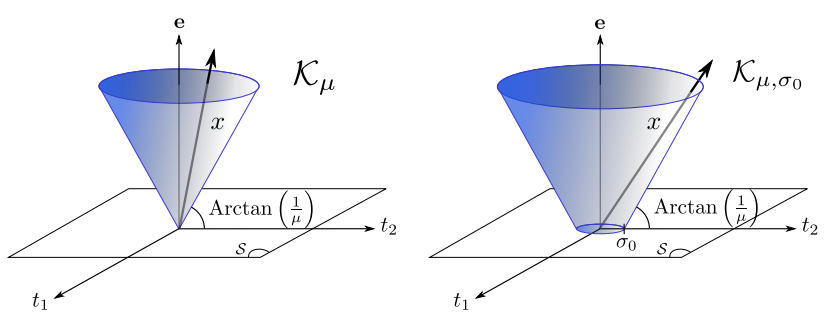

Figure 2: The second-order cone (left) and the truncated second-order cone (right) represented in 3D $(n=3)$.

Now, let us recall the definition of the normal cone to a convex set $\mathcal{C} \subset \mathbb{R}^{n}$ at point $\boldsymbol{x} \in \mathbb{R}^{n}$,

$$
\begin{array}{lr}
\mathcal{N}_{\mathcal{C}}(\boldsymbol{x}):=\left\{\boldsymbol{y} \in \mathbb{R}^{n}, \boldsymbol{y}^{\top}(\boldsymbol{z}-\boldsymbol{x}) \leq 0 \forall \boldsymbol{z} \in \mathcal{C}\right\} & \text { if } \boldsymbol{x} \in \mathcal{C} \\
\mathcal{N}_{\mathcal{C}}(\boldsymbol{x}):=\varnothing & \text { if } \boldsymbol{x} \in \mathbb{R}^{n}-\mathcal{C} .
\end{array}
$$

Note that for an interior point $\boldsymbol{x} \in \operatorname{Int} \mathcal{C}$, we have $\mathcal{N}_{C}(x)=\{0\}$. Moreover, in the particular case when $C$ has a smooth boundary, then on each point $\boldsymbol{x}$ of the boundary $\operatorname{Bd} \mathcal{C}$, the normal cone is spanned by the outward normal $\mathbf{n}_{\mathcal{C}}(\boldsymbol{x})$ to $\mathcal{C}$ at $\boldsymbol{x}$, i.e., $\mathcal{N}_{\mathcal{C}}(\boldsymbol{x} \in \operatorname{Bd} \mathcal{C})=$ $\left\{\alpha \mathbf{n}_{\mathcal{C}}(\boldsymbol{x}), \alpha \in \mathbb{R}_{+}\right\}$.

Let $\xi$ be a positive scalar, $\boldsymbol{x} \in \mathbb{R}^{n}$ and $\boldsymbol{y} \in \mathbb{R}^{n}$. From the projection theorem (see, e.g., [Hiriart-Urruty and Lemaréchal, 2001, Proposition A.5.3.3]), we have

$$
\Pi_{\mathcal{C}}(\boldsymbol{x}-\xi \boldsymbol{y})=\boldsymbol{x} \Longleftrightarrow \boldsymbol{y} \in-\mathcal{N}_{\mathcal{C}}(\boldsymbol{x}) .
$$

In the particular case where $C=\mathcal{K}_{\mu}^{n}$, we have moreover

$$
\boldsymbol{y} \in-\mathcal{N}_{\mathcal{C}}(\boldsymbol{x}) \Longleftrightarrow \mathcal{K}_{\frac{1}{\mu}}^{n} \ni \boldsymbol{y} \perp \boldsymbol{x} \in \mathcal{K}_{\mu}^{n} .
$$

Based on (6), (9) and (10), one may then derive the socalled De Saxcé function satisfying (5),

$$
\begin{aligned}
& f_{\mathrm{DS}}: \mathbb{R}^{d} \times \mathbb{R}^{d} \longrightarrow \quad \mathbb{R}^{d} \\
& (\boldsymbol{r}, \boldsymbol{u}) \longmapsto \Pi_{\mathcal{K}_{\mu}^{d}}(\boldsymbol{r}-\xi \hat{\boldsymbol{u}})-\boldsymbol{r},
\end{aligned}
$$

where $\xi$ is a positive real number.

In Section 3.2.3 we will show that extended versions of both the Alart-Curnier and De Saxcé functions will play a similar role for our continuum granular rheology $\mathcal{D} \mathcal{P}\left(\mu, \sigma_{0}\right)$. But since the above contact problem only involves the canonical Euclidean space $\mathbb{R}^{d}$, we first need to introduce some mathematical tools in order to transpose it to the space of symmetric tensors.

\subsubsection{Preliminary tools}

Recall that $S_{d}$ is the space of $d \times d$ symmetric tensors. Let $\mathfrak{s}(d):=\operatorname{dim} S_{d}=\frac{d(d+1)}{2}$. The dimension of the hyperplane of traceless tensors in $S_{d}$ is $\mathfrak{t}(d):=\mathfrak{s}(d)-1=$ $(d-1)\left(1+\frac{d}{2}\right)$.

Definition 1. Let us introduce the morphism $\chi$,

$$
\begin{array}{rlrl}
\chi: \mathbb{R} \times \mathbb{R}^{\mathrm{t}(d)} & \rightarrow S_{d} & \\
(a ; b, c) & \mapsto\left(\begin{array}{cc}
b & c \\
c & -b
\end{array}\right)+a \mathbf{I} & \text { if } d=2 \\
(a ; b, c, d, e, f) \mapsto & \left(\begin{array}{ccc}
b-\frac{c}{\sqrt{3}} & d & e \\
d & -b-\frac{c}{\sqrt{3}} & f \\
e & f & \frac{2 c}{\sqrt{3}}
\end{array}\right) \\
& +\frac{\sqrt{2}}{\sqrt{3}} a \mathbf{I} & \text { if } d=3 .
\end{array}
$$

The morphism $\chi$ satisfies the following two properties, whose corresponding proofs are given in A.1.

Property 1. $\chi$ is an orthonormal isomorphism between the two Euclidean spaces $\left(\mathbb{R}^{\mathfrak{s}(d)} ; \cdot^{\top}.\right)$ and $\left(S_{d} ;<\cdot, \cdot>\right)$. This means

$$
\forall(\boldsymbol{x}, \boldsymbol{y}) \in \mathbb{R}^{\mathfrak{s}(d)} \times \mathbb{R}^{\mathfrak{s}(d)} \boldsymbol{x}^{\top} \boldsymbol{y}=<\chi(\boldsymbol{x}), \chi(\boldsymbol{y})>
$$

where $\boldsymbol{x}^{\top} \boldsymbol{y}$ is the usual scalar product on $\mathbb{R}^{m}, m \geq 1$ and $<\boldsymbol{\sigma}, \boldsymbol{\tau}\rangle=\frac{\boldsymbol{\sigma}: \boldsymbol{\tau}}{2}$ is our scalar product on $S_{d}$.

Property 2. Let us use the notation $\left(\sigma_{N} ; \boldsymbol{\sigma}_{T}\right):=\overline{\boldsymbol{\sigma}}:=$ $\chi^{-1}(\boldsymbol{\sigma})$ to decompose a symmetric tensor $\boldsymbol{\sigma} \in S_{d}$ or its preimage by $\chi, \overline{\boldsymbol{\sigma}} \in \mathbb{R}^{\mathfrak{s}(d)}$, into a normal component $\sigma_{N} \epsilon$ $\mathbb{R}$ and a tangential component $\boldsymbol{\sigma}_{T} \in \mathbb{R}^{\mathfrak{t}(d)}$. We have

$$
\begin{array}{cc} 
& \frac{1}{\sqrt{2 d}} \operatorname{Tr}(\boldsymbol{\sigma})=\sigma_{N} \\
\text { and } \quad & |\operatorname{Dev}(\boldsymbol{\sigma})|=\left\|\boldsymbol{\sigma}_{T}\right\| \text {. }
\end{array}
$$

\subsubsection{Reformulation of our rheology $\mathcal{D} \mathcal{P}\left(\mu, \sigma_{0}\right)$ on $\mathbb{R}^{\mathfrak{s}(d)}$}

Thanks to our isomorphism $\chi$, our Drucker-Prager rheology $\mathcal{D P}\left(\mu, \sigma_{0}\right)$ (see Section 3.1.4), initially expressed on the tensorial space $S_{d}$, can be reformulated on $R^{\mathfrak{s}(d)}$. The complete proof is given in A.2. 
Theorem 1. Using the notations of Property 2, the rheology $\mathcal{D P}\left(\mu, \sigma_{0}\right)$ can be equivalently expressed as

$$
\begin{cases}\boldsymbol{\lambda}_{T}=-\kappa_{\mu, \boldsymbol{\sigma}_{0}}\left(\lambda_{N}\right) \frac{\mathrm{D}(\mathbf{u})_{T}}{\left\|\mathrm{D}(\mathbf{u})_{T}\right\|} & \text { if } \mathrm{D}(\mathbf{u})_{T} \neq \mathbf{0} \\ \left\|\boldsymbol{\lambda}_{T}\right\| \leq \kappa_{\mu, \boldsymbol{\sigma}_{0}}\left(\lambda_{N}\right) & \text { if } \mathrm{D}(\mathbf{u})_{T}=\mathbf{0}, \\ 0 \leq \lambda_{N} \perp \mathrm{D}(\mathbf{u})_{N} \geq 0, & \end{cases}
$$

that is,

$$
\begin{aligned}
(\boldsymbol{\lambda} ; \mathrm{D}(\mathbf{u})) \in \mathcal{D} \mathcal{P}\left(\mu, \sigma_{0}\right) & \Longleftrightarrow \\
(\bar{\lambda} ; \overline{\mathrm{D}(\mathbf{u})}) & \text { satisfies Problem (13). }
\end{aligned}
$$

Problem (13), which now depends on vector variables, looks very similar to the disjunctive formulation (4) of the Coulomb friction law $\mathcal{C}(\mu)$ between discrete bodies, where $\overline{\boldsymbol{\lambda}}$ plays the role ${ }^{2}$ of the reaction force $\boldsymbol{r}$, and $\overline{\mathrm{D}(\mathbf{u})}$ that of the relative velocity $\boldsymbol{u}$. Actually we shall see in the following section that in the special case where our fluid rheology is considered to be bidimensional $(d=2)$ and where $\sigma_{0}=0$, Problem (13) then becomes exactly equivalent to the tridimensional discrete law $\mathcal{C}(\mu)$.

\subsubsection{Functional formulations of our rheology $\mathcal{D P}\left(\mu, \sigma_{0}\right)$ on $\mathbb{R}^{\mathfrak{s}(d)}$}

In this section, we show that similar functional formulations as those introduced in Section 3.2.1 for the solid contact law $\mathcal{C}(\mu)$ do apply to our fluid rheology $\mathcal{D P}\left(\mu, \sigma_{0}\right)$. The corresponding proofs are given in A.2.

\section{Extended Alart-Curnier formulation}

Definition 2. Let us introduce the following extension of the Alart-Curnier function $f_{A C}^{\star}$,

$$
\begin{aligned}
\mathbb{R}^{\mathfrak{s}(d)} \times \mathbb{R}^{\mathfrak{s}(d)} & \rightarrow \mathbb{R}^{\mathfrak{s}(d)} \\
(\overline{\boldsymbol{\lambda}}, \overline{\mathrm{D}(\mathbf{u})}) & \mapsto\left(\begin{array}{l}
\Pi_{\mathbb{R}_{+}}\left(\lambda_{N}-\xi_{N} \mathrm{D}(\mathbf{u})_{N}\right)-\lambda_{N} \\
\Pi_{\mathcal{B}^{\mathfrak{t}(d)}\left(\kappa\left(\lambda_{N}\right)\right)}\left(\boldsymbol{\lambda}_{T}-\xi_{T} \mathrm{D}(\mathbf{u})_{T}\right)-\boldsymbol{\lambda}_{T}
\end{array}\right)
\end{aligned}
$$

where $\xi_{T}$ and $\xi_{N}$ are positive real numbers, and $\mathcal{B}^{\mathfrak{t}(d)}(a) \subset \mathbb{R}^{\mathfrak{s}(d)}$

$\mathbb{R}^{\mathrm{t}(d)}$ is the ball of radius $a \geq 0$ centered at the origin.

Theorem 2. The following equivalence holds,

$$
(\boldsymbol{\lambda} ; \mathrm{D}(\mathbf{u})) \in \mathcal{D} \mathcal{P}\left(\mu, \sigma_{0}\right) \Longleftrightarrow f_{A C}^{\star}(\overline{\boldsymbol{\lambda}}, \overline{\mathrm{D}(\mathbf{u})})=0 .
$$

Extended De Saxcé formulation Let $n \geq 2$. Let $\mathcal{K}_{\mu, \sigma_{0}}^{n}$ be the truncated version of the $\mathbf{S O C} \mathcal{K}_{\mu}^{n}$ already defined in (7),

$$
\mathcal{K}_{\mu, \sigma_{0}}^{n}:=\left\{\left(x_{\mathrm{N}}, \boldsymbol{x}_{\mathrm{T}}\right) \in \mathbb{R}_{+} \times \mathbb{R}^{n-1},\left\|\boldsymbol{x}_{\mathrm{T}}\right\| \leq \sigma_{0}+\mu x_{\mathrm{N}}\right\} .
$$

See Figure 2, right, for an illustration in the case when $n=3$. Note that similarly to $\mathcal{K}_{\mu}^{n}$, the set $\mathcal{K}_{\mu, \sigma_{0}}^{n}$ is convex, and for $\sigma_{0}=0$ we recover $\mathcal{K}_{\mu, \sigma_{0}}^{n}=\mathcal{K}_{\mu}^{n}$.

\footnotetext{
${ }^{2}$ Note however that the tangential counterparts of $\bar{\lambda}$ and $\boldsymbol{r}$, and of $\overline{\mathrm{D}(\mathbf{u})}$ and $\boldsymbol{u}$ respectively, do not share the same dimension. For instance, when $d=3, \boldsymbol{\lambda}_{\mathrm{T}} \in \mathbb{R}^{5}$ and $\mathrm{D}(\mathbf{u})_{\mathrm{T}} \in \mathbb{R}^{5}$ while $\boldsymbol{r}_{\mathrm{T}} \in \mathbb{R}^{2}$ and $\boldsymbol{u}_{\mathrm{T}} \in \mathbb{R}^{2}$
}

Let us apply a similar change of variable to De Saxcé's [De Saxcé and Feng, 1998] on our symmetric velocity gradient tensor $\mathrm{D}(\mathbf{u}), \quad \hat{\mathrm{D}}(\mathbf{u}):=\mathrm{D}(\mathbf{u})+$ $\sqrt{\frac{2}{d}} \mu|\operatorname{Dev}(\mathrm{D}(\mathbf{u}))| \mathbf{I}$. Using (12) and the linearity of the trace operator on the one hand, the linearity of the ()$_{\mathrm{T}}$ operator and the fact that $\mathbf{I}_{\mathrm{T}}=\mathbf{0}$ on the other hand, we get

$$
\begin{aligned}
& \hat{\mathrm{D}}(\mathbf{u})_{\mathrm{N}}=\mathrm{D}(\mathbf{u})_{\mathrm{N}}+\mu\left\|\mathrm{D}(\mathbf{u})_{\mathrm{T}}\right\| \\
& \hat{\mathrm{D}}(\mathbf{u})_{\mathrm{T}}=\mathrm{D}(\mathbf{u})_{\mathrm{T}} .
\end{aligned}
$$

Similarly to the modified solid velocity $\hat{\boldsymbol{u}} \in \mathbb{R}^{d}$ introduced in Section 3.2.1, which automatically lies in the polar cone $\mathcal{K}_{\frac{1}{\mu}}^{d}$ when $u_{\mathrm{N}} \geq 0$, our quantity $\overline{\hat{\mathrm{D}}(\mathbf{u})} \in \mathbb{R}^{1+d T}$ has an unchanged tangential component and a normal component that is modified such that $\overline{\hat{\mathrm{D}}(\mathbf{u})} \in \mathcal{K}_{\frac{1}{\mu}}^{\mathfrak{s}(d)}$ when $\mathrm{D}(\mathbf{u})_{\mathrm{N}} \geq 0$ (see Equivalence (47a)).

Definition 3. Let us introduce the following extension of the De Saxcé function,

$$
\begin{array}{rllc}
f_{D S}^{\star} \quad: \quad \mathbb{R}^{\mathfrak{s}(d)} \times \mathbb{R}^{\mathfrak{s}(d)} & \longrightarrow & \mathbb{R}^{\mathfrak{s}(d)} \\
& (\overline{\boldsymbol{\lambda}}, \overline{\mathrm{D}(\mathbf{u})}) & \longmapsto & \Pi_{\mathcal{K}_{\mu, \sigma_{0}}^{\mathfrak{s}(d)}}(\overline{\boldsymbol{\lambda}}-\xi \overline{\hat{\mathrm{D}}(\mathbf{u})})-\bar{\lambda}
\end{array}
$$

where $\xi$ is a positive real number.

Theorem 3. The following equivalence holds,

$$
(\boldsymbol{\lambda} ; \mathrm{D}(\mathbf{u})) \in \mathcal{D} \mathcal{P}\left(\mu, \sigma_{0}\right) \Longleftrightarrow f_{D S}^{\star}(\overline{\boldsymbol{\lambda}}, \overline{\mathrm{D}(\mathbf{u})})=\mathbf{0} .
$$

Corollary 1. When $\sigma_{0}=0$, the rheology $\mathcal{D} \mathcal{P}\left(\mu, \sigma_{0}\right)$ can be expressed as a Second-Order Cone Complementarity Problem (SOCCP),

$$
(\lambda ; \mathrm{D}(\mathbf{u})) \in \mathcal{D} \mathcal{P}(\mu, 0) \Longleftrightarrow \mathcal{K}_{\mu}^{\mathfrak{s}(d)} \ni \bar{\lambda} \perp \overline{\hat{\mathrm{D}}(\mathbf{u})} \in \mathcal{K}_{\frac{1}{\mu}}^{\mathfrak{s}(d)}
$$

where the $\perp$ notation refers to Euclidean orthogonality in

\section{Creeping flow}

\subsection{The continuous setting}

In this first section we assume that the flow is slow enough for its inertia to be neglected, and solve for its steady state. This case is relevant since the structure of the equations for each time-step of the fully dynamic case will be similar (see Section 5).

Let us consider a domain $\Omega \subset \mathbb{R}^{d}$ and decompose its boundary as $\partial \Omega:=B_{D} \cup B_{N}$, with Dirichlet boundary conditions on $B_{D}$ and homogeneous Neumann on $B_{N}$,

$$
\begin{aligned}
\mathbf{u} & =\mathbf{u}_{D} & & \text { on } B_{D} \\
\mathrm{D}^{b}(\mathbf{u}) \mathbf{n}_{\Omega} & =\mathbf{0} & & \text { on } B_{N}
\end{aligned}
$$

where $\mathbf{n}_{\Omega}$ is the outward-pointing normal to $\Omega$ on each point of its boundary. 
Moreover, let $\boldsymbol{\sigma}_{\text {ext }}$ gather all external stresses applied onto the Neumann boundary of the domain. We thus have

$$
-\boldsymbol{\lambda} \mathbf{n}_{\Omega}=\sigma_{\text {ext }} \mathbf{n}_{\Omega}
$$$$
\text { on } B_{N}
$$

Conservation of momentum gives

$$
-\nabla \cdot[\underbrace{2 \eta \mathrm{D}^{b}(\mathbf{u})-\boldsymbol{\lambda}}_{=\boldsymbol{\sigma}_{\text {tot }}}]=\rho g \mathbf{e}_{g} \quad \text { on } \Omega
$$

with $\mathbf{e}_{g}$ the "down" unit vector.

Dimensionless equations Let $L$ be a characteristic dimension of the flow. We define $U:=\sqrt{g L}$ the characteristic velocity and $P:=\rho g L$ the characteristic pressure of the flow. We consider dimensionless differential operators defined through $\tilde{\nabla}:=L \nabla$. We furthermore introduce two dimensionless numbers, the Reynolds number Re $:=\frac{\rho U L}{\eta}$ and the Bingham number $\mathrm{Bi}:=\frac{\sigma_{0}}{\rho g L}=\frac{\sigma_{0}}{P}$.

Considering the dimensionless quantities $\tilde{\mathbf{u}}:=\frac{1}{U} \mathbf{u}, \tilde{\boldsymbol{\tau}}:=$ $\frac{1}{P} \boldsymbol{\tau}, \tilde{\boldsymbol{\lambda}}:=\frac{1}{P} \boldsymbol{\lambda}$, and $\tilde{\mathbf{u}}_{D}:=\frac{1}{U} \mathbf{u}_{D}$, Equations $(20-22)$ can be made dimensionless as

$$
\left\{\begin{aligned}
-\tilde{\nabla} \cdot\left[\frac{2}{\operatorname{Re}} \widetilde{\mathrm{D}^{b}}(\tilde{\mathbf{u}})-\tilde{\boldsymbol{\lambda}}\right] & =\mathbf{e}_{g} & & \text { on } \Omega \\
\tilde{\mathbf{u}} & =\tilde{\mathbf{u}}_{D} & & \text { on } B_{D} \\
\widetilde{\mathrm{D}^{b}}(\tilde{\mathbf{u}}) \mathbf{n}_{\Omega} & =\mathbf{0} & & \text { on } B_{N} \\
-\tilde{\boldsymbol{\lambda}} \mathbf{n}_{\Omega} & =\tilde{\boldsymbol{\sigma}}_{e x t} \mathbf{n}_{\Omega} & & \text { on } B_{N}
\end{aligned}\right.
$$

with the dimensionless rheology

$$
(\tilde{\boldsymbol{\lambda}} ; \tilde{\mathrm{D}}(\tilde{\mathbf{u}})) \in \mathcal{D} \mathcal{P}(\mu, \mathrm{Bi})
$$

Proof. By noting that $\tilde{\mathrm{D}}(\tilde{\mathbf{u}})=\frac{L}{U} \mathrm{D}(\mathbf{u})$ and $\frac{2 \eta U}{L^{2} \rho g}=\frac{2}{\operatorname{Re}}$, Equations $(23)$ can be obtained from $(20-22)$ by direct calculation. As for the dimensionless granular rheology (24), it directly follows from (13) by noting that $\frac{1}{P} \kappa_{\mu, \sigma_{0}}\left(\lambda_{\mathrm{N}}\right)=\kappa_{\mu, \mathrm{Bi}}\left(\tilde{\lambda}_{\mathrm{N}}\right)$, and that, again, complementarity is insensitive to positive scaling factors.

In the remainder of this article, unless otherwise mentioned we shall use the dimensionless quantities and omit the tildes. In particular, from now we shall use the notation $(\boldsymbol{\lambda} ; \mathrm{D}(\mathbf{u})) \in \mathcal{D} \mathcal{P}(\mu, \mathrm{Bi})$ for denoting our granular rheology.

\subsection{Variational formulation}

Let $H^{1}(\Omega)^{d}$ be the usual Sobolev space containing square-integrable functions from $\mathbb{R}^{d} \times \mathbb{R}$ to $\mathbb{R}^{d}$, with square-integrable gradients. As in [Saramito, 2015, Appendix A], we introduce the subspace $V\left(\mathbf{u}_{D}\right)$ of $H^{1}(\Omega)^{d}$ for which the Dirichlet boundary condition (20a) is satisfied, i.e.,

$$
V\left(\mathbf{u}_{D}\right)=\left\{\mathbf{u} \in H^{1}(\Omega)^{d} ; \mathbf{u}=\mathbf{u}_{D} \text { on } B_{D}\right\}
$$

and the subspace $V(\mathbf{0})$ of $H^{1}(\Omega)^{d}$ for which the homogeneous Dirichlet boundary condition is satisfied, i.e.,

$$
V(\mathbf{0})=\left\{\mathbf{v} \in H^{1}(\Omega)^{d} ; \mathbf{v}=\mathbf{0} \text { on } B_{D}\right\} .
$$

Let $T(\Omega)$ be the space of square-integrable symmetric tensor fields on $\Omega$.

Proposition 1. A weak form of System (23 - 24) amounts to finding $\mathbf{u} \in V\left(\mathbf{u}_{D}\right), \boldsymbol{\lambda} \in T(\Omega)$, and $\boldsymbol{\gamma} \in T(\Omega)$, such that

$$
\left\{\begin{aligned}
a(\mathbf{u}, \mathbf{v}) & =b(\boldsymbol{\lambda}, \mathbf{v})+l(\mathbf{v}) & & \forall \mathbf{v} \in V(\mathbf{0}) \\
m(\boldsymbol{\gamma}, \boldsymbol{\tau}) & =b(\boldsymbol{\tau}, \mathbf{u}) & & \forall \boldsymbol{\tau} \in T(\Omega) \\
(\boldsymbol{\lambda} ; \boldsymbol{\gamma}) & \in \mathcal{D} \mathcal{P}(\mu, \mathrm{Bi}), & &
\end{aligned}\right.
$$

where $\forall \mathbf{x}, \mathbf{y} \in H^{1}(\Omega)^{d}$ and $\forall \boldsymbol{\sigma}, \boldsymbol{\tau} \in T(\Omega), a(\mathbf{x}, \mathbf{y})$ and $m(\boldsymbol{\sigma}, \boldsymbol{\tau})$ are the symmetric positive-definite bilinear forms on $H^{1}(\Omega)^{d} \times H^{1}(\Omega)^{d}$ and $T(\Omega) \times T(\Omega)$, respectively,

$$
\begin{aligned}
a(\mathbf{x}, \mathbf{y}) & =\frac{2}{\operatorname{Re}} \int_{\Omega} \mathrm{D}^{0}(\mathbf{x}): \mathrm{D}^{0}(\mathbf{y})+\frac{b}{d}(\nabla \cdot \mathbf{x})(\nabla \cdot \mathbf{y}) \\
m(\boldsymbol{\sigma}, \boldsymbol{\tau}) & =\int_{\Omega} \boldsymbol{\sigma}: \boldsymbol{\tau},
\end{aligned}
$$

$b(\boldsymbol{\tau}, \mathbf{x})$ is the bilinear form on $T(\Omega) \times H^{1}(\Omega)^{d}$,

$$
b(\tau, \mathbf{x})=\int_{\Omega} \mathrm{D}(\mathbf{x}): \tau,
$$

and $l(\mathbf{x})$ is the linear form on $H^{1}(\Omega)^{d}$,

$$
l(\mathbf{x})=\int_{\Omega} \mathbf{e}_{g} \cdot \mathbf{x}-\int_{B_{N}}\left(\boldsymbol{\sigma}_{e x t} \mathbf{n}_{\Omega}\right) \cdot \mathbf{x}
$$

Proof. First, let us consider the stress boundary condition (21). One solution would be to enforce it strongly, by discretizing $\boldsymbol{\lambda}$ over the subspace of $T(\Omega)$ which satisfy (21). However, this may lead to difficulties in the discretization of the $\mathcal{D} \mathcal{P}(\mu, \mathrm{Bi})$ rheology. In our proposed implementation, we chose instead to allow in the integration of the term $\nabla \cdot \boldsymbol{\sigma}_{\text {tot }}$ for a possibly non-zero jump $\llbracket \boldsymbol{\sigma}_{t o t} \rrbracket$ of the stress on $B_{N}$.

Let us now derive the variational formulation for System (23). We assume $\mathbf{u} \in V\left(\mathbf{u}_{D}\right)$, and $\boldsymbol{\lambda} \in T\left(\boldsymbol{\sigma}_{\text {ext }}\right)$, and let $\mathbf{v} \in V(\mathbf{0})$ be a test function. In this setting, multiplying both sides of (23) by $\mathbf{v}$ and integrating over $\Omega$ yields

$$
-\int_{\Omega} \nabla \cdot\left(\frac{2}{\operatorname{Re}} \mathrm{D}^{b}(\mathbf{u})-\boldsymbol{\lambda}\right) \cdot \mathbf{v}+\int_{B_{N}} \llbracket \boldsymbol{\sigma}_{t o t} \rrbracket \mathbf{n}_{\Omega} \cdot \mathbf{v}=\int_{\Omega} \mathbf{e}_{g} \cdot \mathbf{v} .
$$

Using the Green formula with the (20b) boundary condition for $\mathbf{u}$ and the homogeneous Dirichlet condition for $\mathbf{v}$

$$
\begin{aligned}
a(\mathbf{u}, \mathbf{v}) & =\frac{2}{\operatorname{Re}} \int_{\Omega} \mathrm{D}^{0}(\mathbf{u}): \mathrm{D}^{0}(\mathbf{v})+\frac{b}{d}(\nabla \cdot \mathbf{v})(\nabla \cdot \mathbf{u}) \\
& =\int_{\Omega} \frac{2}{\operatorname{Re}} \mathrm{D}^{b}(\mathbf{u}): \mathrm{D}(\mathbf{v}) \\
& =-\int_{\Omega}\left(\nabla \cdot \frac{2}{\operatorname{Re}} \mathrm{D}^{b}(\mathbf{u})\right) \cdot \mathbf{v}
\end{aligned}
$$


and

$$
\begin{aligned}
& \int_{\Omega} \nabla \cdot[\boldsymbol{\lambda}] \cdot \mathbf{v}+\int_{B_{N}} \llbracket \boldsymbol{\sigma}_{t o t} \rrbracket \mathbf{n}_{\Omega} \cdot \mathbf{v}=-\int_{B_{N}} \boldsymbol{\sigma}_{e x t} \mathbf{n}_{\Omega} \cdot \mathbf{v} \\
& -b(\boldsymbol{\lambda}, \mathbf{v}) \text {. }
\end{aligned}
$$

By combining $(26-28)$ and the definition of $l$, we retrieve (25a).

Let us now focus on the rheology $\mathcal{D} \mathcal{P}(\mu, \mathrm{Bi})$ given in $(24)$, which contains inequalities that cannot be put directly under weak form. To circumvent this difficulty, we introduce an auxiliary variable $\gamma \in T(\Omega)$ that weakly satisfies $\gamma=\mathrm{D}(\mathbf{u})$, i.e.,

$$
\int_{\Omega} \mathrm{D}(\mathbf{u}): \tau=\int_{\Omega} \gamma: \tau \quad \forall \boldsymbol{\tau} \in T(\Omega)
$$

which is exactly equation (25b). We can thus express the rheology $\mathcal{D P}(\mu, \mathrm{Bi})$ under the weak form as $(25 \mathrm{~b}-$ $25 \mathrm{c})$.

Remark Note that we do not include any additional equation ensuring the well-posedness of our system, such as the zero-average pressure condition which is commonly used for Stokes flows. Indeed, for a given velocity field, the $\mathcal{D P}(\mu, \mathrm{Bi})$ rheology imposes the value of $\boldsymbol{\lambda}$ in the yielded regions, serving as an intrinsic boundary condition for the stress field inside the rigid zones.

\subsection{Discretization by Finite Elements (FEM)}

\subsubsection{Discretization of the symmetric tensor fields}

For the discretization of the space $T(\Omega)$, we shall make use of Lagrange FEM, that is all symmetric tensor fields will be expressed as functions of $\Omega$, built as an interpolation of their values at the $n$ degrees of freedom $x_{i}$.

Let $Q_{h}$ be a finite-dimensional subspace of $L_{2}(\Omega)$ of dimension $n$. Let $\left(\alpha_{i}\right)_{1 \leq i \leq n}$ be a basis of $Q_{h}$, such that $\alpha_{i}\left(x_{j}\right)=\delta_{i, j}$. Let $\left(\mathbf{e}_{j}\right)_{1 \leq j \leq \mathfrak{s}(d)}$ be the canonical basis for $\mathbb{R}^{\mathfrak{s}(d)}$. Then $\left(\boldsymbol{\alpha}_{k}^{\mathfrak{s}(d)}\right)_{1 \leq k \leq \mathfrak{s}(d) n}$, defined as

$\forall x \in \Omega, \quad \boldsymbol{\alpha}_{\mathfrak{s}(d)(i-1)+j}^{\mathfrak{s}(d)}(x):=\alpha_{i}(x) \mathbf{e}_{j} \quad$ for $\left\{\begin{array}{l}1 \leq i \leq n \\ 1 \leq j \leq \mathfrak{s}(d)\end{array}\right.$

is a natural basis for $Q_{h}^{\mathfrak{s}(d)}$. Finally, we may build a finite subspace $T_{h} \subset T(\Omega)$ from the basis $\left(\sigma_{k}\right)_{1 \leq k \leq \mathfrak{s}(d) n}$ defined as

$\forall x \in \Omega, \quad \sigma_{\mathfrak{s}(d)(i-1)+j}(x):=\alpha_{i}(x) \chi\left(\mathbf{e}_{j}\right) \quad$ for $\left\{\begin{array}{l}1 \leq i \leq n \\ 1 \leq j \leq \mathfrak{s}(d)\end{array}\right.$

where our orthogonal isomorphism $\chi$ from $\mathbb{R}^{\mathfrak{s}(d)}$ to $S_{d}$ has been formerly introduced in Definition 1 . We have the following relationship between the two bases $\left(\boldsymbol{\alpha}^{\mathfrak{s}(d)}\right)_{k}$ and $(\sigma)_{k}$,

$$
\forall x \in \Omega, \quad \chi\left(\boldsymbol{\alpha}_{k}^{\mathfrak{s}(d)}(x)\right)=\boldsymbol{\sigma}_{k}(x) \quad \text { for } 1 \leq k \leq \mathfrak{s}(d) n .
$$

Now, for $\boldsymbol{\lambda}$ and $\boldsymbol{\gamma}$ two symmetric tensor fields in $T(\Omega)$, let $\lambda_{h}, \gamma_{h} \in T_{h}$ be their corresponding discretized functions built by interpolation at the $n$ degrees of freedom $x_{i}$. Let $\boldsymbol{\Lambda}_{h}$ and $\boldsymbol{\Gamma}_{h}$ be the vectors of the (scalar) coefficients of the decomposition of $\boldsymbol{\lambda}_{h}$ and $\gamma_{h}$, respectively, in the basis $(\boldsymbol{\sigma})_{k}$. That is, $\boldsymbol{\lambda}_{h}(x)=\sum_{k} \boldsymbol{\Lambda}_{h, k} \boldsymbol{\sigma}_{k}(x)$, and $\gamma_{h}(x)=\sum_{k} \boldsymbol{\Gamma}_{h, k} \boldsymbol{\sigma}_{k}(x)$. Note that using (30), functions $\overline{\boldsymbol{\lambda}_{h}}$ and $\overline{\gamma_{h}}$ decompose on the basis $\left(\boldsymbol{\alpha}^{\mathfrak{s}(d)}\right)_{k}$ with exactly the same coefficients, that is $\overline{\boldsymbol{\lambda}_{h}}(x)=$ $\sum_{k} \boldsymbol{\Lambda}_{h, k} \boldsymbol{\alpha}_{k}^{\mathfrak{s}(d)}(x)$, and $\overline{\gamma_{h}}(x)=\sum_{k} \boldsymbol{\Gamma}_{h, k} \boldsymbol{\alpha}_{k}^{\mathfrak{s}(d)}(x)$. Thus, since $\boldsymbol{\alpha}_{\mathfrak{s}(d)(m-1)+j}^{\mathfrak{s}(d)}\left(x_{i}\right)=\delta_{i, m} \mathbf{e}_{j}$, each vector of $\mathfrak{s}(d)$ coefficients $\boldsymbol{\Lambda}_{h}[i]:=\left\{\boldsymbol{\Lambda}_{h, \mathfrak{s}(d)(i-1)+j}, 1 \leq j \leq \mathfrak{s}(d)\right\}$ corresponds to the value $\overline{\boldsymbol{\lambda}_{h}}\left(x_{i}\right)$, and the decomposition of $\overline{\boldsymbol{\lambda}_{h}}(x)$ on the scalar basis $(\alpha)_{i}$ reads $\overline{\boldsymbol{\lambda}_{h}}(x)=\sum_{i} \boldsymbol{\Lambda}_{h}[i] \alpha_{i}(x)$. Similarly, we have $\Gamma_{h}[i]=\overline{\gamma_{h}}\left(x_{i}\right)$ and $\overline{\gamma_{h}}(x)=\sum_{i} \Gamma_{h}[i] \alpha_{i}(x)$.

From (19) and using the aforementioned properties, we may express our discrete granular rheology at each degree of freedom $x_{i, 1 \leq i \leq n}$ as

$$
\left(\boldsymbol{\lambda}_{h}\left(x_{i}\right) ; \gamma_{h}\left(x_{i}\right)\right) \in \mathcal{D P}(\mu, \mathrm{Bi}) \Longleftrightarrow f_{\mathrm{DS}}^{\star}\left(\Lambda_{h}[i], \boldsymbol{\Gamma}_{h}[i]\right)=\mathbf{0} .
$$

\subsubsection{Discretization of the (bi)linear forms}

Let $U_{h} \subset H^{1}(\Omega)^{d}$ be a finite-dimensional vectorial space, and $V_{h} \subset U_{h}$ its subspace satisfying the Dirichlet boundary conditions

$$
V_{h}\left(\mathbf{u}_{D}\right):=\left\{\mathbf{v}_{h} \in U_{h}, \mathbf{v}_{h}=\mathbf{u}_{D h} \text { on } B_{D}\right\}
$$

where $\mathbf{u}_{D h}$ is the approximated (discrete) value of $\mathbf{u}_{D}$ on the FEM mesh. Let $\mathbf{u}_{D h}^{*} \in U_{h}$ be the unique function such that for any $\mathbf{u}_{h} \in V_{h}\left(\mathbf{u}_{D}\right), \mathbf{u}_{D h}^{*}=\mathbf{u}_{h}-\Pi_{V_{h}(\mathbf{0})}\left(\mathbf{u}_{h}\right)$, where we recall that $\Pi_{\mathcal{C}}$ is the orthogonal projection operator onto the convex set $\mathcal{C}$.

Unlike for the space $T_{h}$, here we make no specific assumption regarding the structure of $V_{h}\left(\mathbf{u}_{D}\right)$ or the construction of a corresponding base. Let $\mathfrak{v}:=\operatorname{dim} V_{h}$. Given $\left(\boldsymbol{v}_{i}\right)_{1 \leq i \leq \mathfrak{v}}$ a basis of $V_{h}(\mathbf{0})$, we denote by $A, B$, and $M$ the matrices corresponding to the decomposition of the bilinear forms $a, b$, and $m$ respectively, and by $\mathbf{l}$ the vector corresponding to the decomposition of the linear form $l$. More precisely, we have $A_{i, j}=a\left(\boldsymbol{v}_{i}, \boldsymbol{v}_{j}\right)$, $M_{k, \ell}=m\left(\boldsymbol{\sigma}_{k}, \boldsymbol{\sigma}_{\ell}\right), B_{k, j}=b\left(\boldsymbol{\sigma}_{k}, \boldsymbol{v}_{j}\right)$ and $\mathbf{l}_{j}=l\left(\boldsymbol{v}_{j}\right)$. Similarly, let $\mathbf{U}_{h}$ be the vector of scalar coefficients corresponding to the decomposition of $\mathbf{u}_{h}$. We have, $\forall x \in \Omega$, $\mathbf{u}_{h}(x)=\sum_{1 \leq i \leq \mathfrak{v}} \mathbf{U}_{h, i} \boldsymbol{v}_{i}(x)+\mathbf{u}_{D h}^{*}(x)$. 


\subsubsection{FEM discrete system}

Finally, at this point the discrete version of Equations $(25 \mathrm{a}-25 \mathrm{c})$ reads: Find $\mathbf{u}_{h} \in V_{h}\left(\mathbf{u}_{D}\right),\left(\boldsymbol{\lambda}_{h}, \gamma_{h}\right) \in T_{h}^{2}$,

$$
\left\{\begin{aligned}
A \mathbf{U}_{h} & =B^{\top} \Lambda_{h}+\underbrace{\mathbf{l}-\mathbf{a}_{D}}_{\mathbf{l}_{t o t}} \\
M \boldsymbol{\Gamma}_{h} & =B \mathbf{U}_{h}+\mathbf{k} \\
\left(\boldsymbol{\lambda}_{h} ; \gamma_{h}\right) & \in \mathcal{D} \mathcal{P}(\mu, \mathrm{Bi})
\end{aligned}\right.
$$

where for $1 \leq i \leq \mathfrak{v}, \mathbf{a}_{D, i}=a\left(\mathbf{u}_{D h}^{*}, \boldsymbol{v}_{i}\right)$ and for $1 \leq k \leq$ $\mathfrak{s}(d) n, \mathbf{k}_{k}=b\left(\boldsymbol{\sigma}_{k}, \mathbf{u}_{D h}^{*}\right)$.

\subsubsection{Discretization of the rheology on quadra- ture points}

In the discrete case, the nonsmooth rheology $\mathcal{D} \mathcal{P}(\mu, \mathrm{Bi})$ will not hold at every point $x \in \Omega$, but only in the weak sense, i.e., on the space $T_{h}$. That is, we replace (32c) with

$$
\int_{\Omega} f_{\mathrm{DS}}^{\star}(\boldsymbol{\lambda}, \gamma): \boldsymbol{\tau}=0 \quad \forall \boldsymbol{\tau} \in T_{h} .
$$

We shall thus restrict ourselves to a discrete number of points $\breve{x}_{j}$ on which to enforce $\left(\boldsymbol{\lambda}_{h}\left(\breve{x}_{j}\right) ; \gamma_{h}\left(\breve{x}_{j}\right)\right) \epsilon$ $\mathcal{D P}(\mu, \mathrm{Bi})$. The remaining question is how to perform a good choice for this set of points $\breve{x}_{j}$. In the sequel we shall see that this choice has a direct impact onto the final form of the system to be solved, and thus on both the physical relevance of the discrete problem and the practical performance of available solving methods.

Discretization on $Q_{h}$ 's Lagrange degrees of freedom One obvious choice for $(\breve{x})_{j}$ is to consider the $n$ degrees of freedom $x_{i}$ themselves, which served to define the $(\alpha)_{i}$ basis and thus the finite-dimensional spaces $Q_{h}$ and $T_{h}$. This way, using (31) we may replace (33) with

$$
f_{\mathrm{DS}}^{\star}\left(\boldsymbol{\Lambda}_{h}[i], \boldsymbol{\Gamma}_{h}[i]\right)=\mathbf{0} \quad \text { for } \quad 1 \leq i \leq n .
$$

Since $A$ and $M$ are positive-definite, we may first eliminate the velocity variable $\mathbf{U}_{h}$ from (32a), and then get a linear relationship between $\Lambda_{h}$ and $\Gamma_{h}$ from (32b), $\boldsymbol{\Gamma}_{h} \propto M^{-1} W \Lambda_{h}$ where $W=B A^{-1} B^{\top}$. Apart from the presence of the matrix $M$ which yields a modified Delassus operator $\tilde{W}:=M^{-1} W$, we have thus obtained a system which is very similar to the one appearing in discrete contact mechanics, and for which a plethora of efficient solving methods exist [Cadoux, 2009]. However, the presence of the matrix $M$ breaks the symmetry of the operator $\tilde{W}$ ( $M$ and $W$ do not commute in the general case). The discrete system (32) therefore lacks a fundamental symmetry property, which is key not only to guarantee physical consistency of our model, but also to design an efficient numerical solver.

From a physical point of view, such an asymmetry in our discrete frictional contact law typically implies that the maximum dissipation principle cannot be satisfied, meaning that some anisotropy is artificially introduced through the discretization.
From a purely numerical point of view, symmetry of the Delassus operator is not necessarily a prerequisite to common numerical solvers, but in our case it proves to be highly desirable for coming up with a tractable solving method. Indeed, among scalable available solvers, we basically have the choice between operator-splitting algorithms (such as the well-known nonsmooth contact dynamics (NSCD) method [Jourdan et al., 1998]), and optimizationbased algorithms (e.g. [Renouf and Alart, 2005, Cadoux, 2009]). On the one hand, operator-splitting methods do not assume $\tilde{W}$ be symmetric, yet for efficiency purposes they require the explicit knowledge of $\tilde{W}$, especially when large systems are involved as it is the case here. In our case $A^{-1}$ is dense, and so is $\tilde{W}$. Making such an explicit assembly thus turns out to be intractable. On the other hand, optimization-based methods do not require the explicit assembly of $\tilde{W}$, however they heavily rely upon its symmetry so as to identify $\Gamma_{h}$ as the gradient of a quadratic function in $\Lambda_{h}$ with Hessian $\tilde{W}$.

For these two reasons, we choose to discretize our constraints on an alternative set of points $(\breve{x})_{j}$ that will allow us to eliminate the matrix $M$ and thus retrieve symmetry of $\tilde{W}$. This way we shall both recover physical consistency of our model, and benefit from efficient optimization-based solving methods.

Discretization on quadrature points As we are using a Lagrange FEM discretization of the space $T(\Omega)$ with polynomial interpolating bases, each integral $M_{k, \ell}=$ $\int \sigma_{k}: \sigma_{\ell}$ can be computed exactly using Gaussian quadrature $^{3}$, i.e., as $\sum_{q} w_{q}\left(\boldsymbol{\sigma}_{k}\left(\hat{x}_{q}\right): \boldsymbol{\sigma}_{\ell}\left(\hat{x}_{q}\right)\right)$ where the $\hat{x}_{q, 1 \leq j \leq n_{Q}}$ are the so-called quadrature points and $w_{q}$ their corresponding weights. As shown below, defining the set $(\breve{x})_{q}$ as the set of quadrature points $\hat{x}_{q}$ allows us to retrieve a symmetric Delassus operator.

Recall that for $\boldsymbol{\lambda}$ a symmetric tensor field in $T(\Omega), \boldsymbol{\lambda}_{h} \epsilon$ $T_{h}$ corresponds to its discretized version interpolating the values at the $n$ degrees of freedom $x_{m}$, with $\overline{\lambda_{h}}\left(x_{m}\right)=$ $\boldsymbol{\Lambda}_{h}[m]$. Let $R$ be the $\left(\mathfrak{s}(d) n_{Q} \times \mathfrak{s}(d) n\right)$ matrix mapping those $\boldsymbol{\Lambda}_{h}[m]$ to the interpolated values $\overline{\boldsymbol{\lambda}_{h}}\left(\breve{x}_{q}\right)$ at the quadrature points $\breve{x}_{q}$. That is, $R$ is such that

$$
\breve{\Lambda}_{h}=R \boldsymbol{\Lambda}_{h}
$$

where $\breve{\Lambda}_{h}$ is the vector of size $\mathfrak{s}(d) n_{Q}$ concatenating the $n_{Q}$ vector values $\breve{\Lambda}_{h}[q]:=\overline{\lambda_{h}}\left(\breve{x}_{q}\right)$ for $1 \leq q \leq n_{Q}$, and $\Lambda_{h}$ the vector of size $\mathfrak{s}(d) n$ concatenating the $n$ vector values $\boldsymbol{\Lambda}_{h}[m]=\overline{\boldsymbol{\lambda}_{h}}\left(x_{m}\right)$ for $1 \leq m \leq n$.

Matrix $R$ contains $n_{Q} \times n$ square blocks $\mathfrak{R}_{q, j}$ of size $\mathfrak{s}(d) \times \mathfrak{s}(d)$ with $\mathfrak{R}_{q, j}=\alpha_{j}\left(\breve{x}_{q}\right) I_{\mathfrak{s}(d)}$ for all $1 \leq q \leq n_{Q}$ and $1 \leq j \leq n$, where $I_{\mathfrak{s}}(d)$ is the $\mathfrak{s}(d) \times \mathfrak{s}(d)$ identity block. This translates into the following elementwise expression $R_{(q-1) \mathfrak{s}(d)+p,(j-1) \mathfrak{s}(d)+\ell}=\alpha_{j}\left(\breve{x}_{q}\right) \delta_{p, \ell} \quad$ for $\quad 1 \leq p, \ell \leq \mathfrak{s}(d)$.

${ }^{3}$ Of course each coefficient $M_{k, \ell}$ may alternatively be evaluated by direct integration, since primitives of the integrand are easily calculable. However, the quadrature technique is mentioned here as it provides a judicious set of points on which to discretize our rheology. 
Property 3. Let $\breve{\Lambda}_{h}:=R \boldsymbol{\Lambda}_{h}$ and $\breve{\Gamma}_{h}:=R \Gamma_{h}$, and $R^{\dagger}$ the Moore-Penrose pseudoinverse of $R$. Then Equations (25a - 25c) can be discretized as:

Find $\mathbf{U}_{h} \in \mathbb{R}^{\mathfrak{v}}, \breve{\Lambda}_{h}, \breve{\Gamma}_{h} \in \mathbb{R}^{\mathfrak{s}(d) n_{Q}}$,

$$
\left\{\begin{array}{c}
A \mathbf{U}_{h}=B^{\top} R^{\dagger} \breve{\Lambda}_{h}+\mathbf{l}_{t o t} \\
\breve{\Gamma}_{h}=R^{\dagger, \top} B \mathbf{U}_{h}+R^{\dagger, \top} \mathbf{k}_{t o t} \\
\forall 1 \leq q \leq n_{Q}, \quad f_{D S}^{\star}\left(\breve{\Lambda}_{h}[q], \breve{\Gamma}_{h}[q]\right)=\mathbf{0} .
\end{array}\right.
$$

The corresponding proof is given in A.3.

This time we have obtained in (34) a system which preserves the symmetry of the new Delassus operator $\breve{W}=$ $R^{\dagger, \top} B A^{-1} B^{\top} R^{\dagger}$. One remaining difficulty stems from the presence of the matrix $R^{\dagger}$, which in the general case could increase substantially the cost of solving the system.

\subsubsection{Considerations on $R^{\dagger}$}

Trapezoidal quadrature rule The first observation is that if the quadrature points $\left(\breve{x}_{q}\right)$ were to coincide with the degrees of freedom $\left(x_{i}\right), R$ would boil down to the identity matrix and the operator $R^{\dagger}$ would not induce any additional cost. This is actually always the case for a piecewise constant $\left(\mathbb{P}_{0}\right)$ approximation, for which both the degrees of freedom and the quadrature points are located at the barycenter of each element.

For higher-order polynomial basis functions, having the $(\breve{x})_{q}$ coincide with the $(x)_{i}$ amounts to computing $m(\boldsymbol{\gamma}, \boldsymbol{\tau})$ using a trapezoidal integration rule. Obviously, such an approximation induces a loss of precision - the integral being exact only for functions that are linear between the degrees of freedom. This means that the order of convergence will not increase with that of the basis functions, and using high-order discretization space $\left(\mathbb{P}_{2}\right.$ or higher-order polynomials) would be wasteful. However, for piecewise linear $\left(\mathbb{P}_{1}\right)$ polynomials, we found such approximation to be acceptable, and used it in practice.

Mixed finite elements In the case of piecewisepolynomial discontinuous basis functions, degrees of freedom are not shared between adjacent elements. When considering such a discretization of the space $T(\Omega)$, the matrix $R$ becomes block-diagonal, and consequently its pseudo-inverse has a similar structure and is easy to compute. The additional cost induced by the presence of the linear operator $R^{\dagger}$ in Problem (34) is therefore once again negligible.

\subsubsection{Final discrete system}

For brevity of notation and since there are no more ambiguities, from now on we shall drop the decorations and capitalization of the variables, i.e., we shall consider Problem (34) written as:
Find $\mathbf{u} \in \mathbb{R}^{\mathfrak{v}}, \boldsymbol{\lambda}, \boldsymbol{\gamma} \in \mathbb{R}^{\mathfrak{s}(d) n_{Q}}$,

$$
\left\{\begin{aligned}
A \mathbf{u} & =B^{\top} R^{\dagger} \boldsymbol{\lambda}+\mathbf{l} \\
\gamma & =R^{\dagger, \top} B \mathbf{u}+R^{\dagger, \top} \mathbf{k} \\
\mathbf{0} & =f_{\mathrm{DS}}^{\star}(\boldsymbol{\lambda}, \boldsymbol{\gamma})
\end{aligned}\right.
$$

where $f_{\mathrm{DS}}^{\star}$ is extended from $\mathbb{R}^{\mathfrak{s}(d)}$ to $\mathbb{R}^{n_{Q} \mathfrak{s}(d)}$ by concatenation.

\subsection{Solving (35) numerically in the case $\mathrm{Bi}=0$}

In the case when $\mathrm{Bi}=0$, from Corollary 1 the constraint (35c) boils down to $\mathcal{K}_{\frac{1}{\mu}} \ni \hat{\gamma} \perp \boldsymbol{\lambda} \in \mathcal{K}_{\mu}$. The resulting problem was extensively studied in the contact mechanics field, see e.g., [Cadoux, 2009] for a review.

\subsubsection{Cadoux's algorithm}

Several methods have been proposed to decompose Problem (35) as a succession of optimization problems depending on a varying parameter $s$, see e.g. [Haslinger and Tvrdỳ, 1983, Renouf and Alart, 2005, Acary et al., 2011]. We recall in this section the fixed-point algorithm originally presented in [Cadoux, 2009, Acary et al., 2011], which allows us to rewrite our problem as a nested loop of Second-Order Cone Quadratic Problems (SOCQP). The advantage of this algorithm over most other iterative approaches is that the successive values of the parameter $s$ depend only on the primal variable $\mathbf{u}$; each intermediate SOCQP will have a unique solution in $\mathbf{u}$, but may admit a continuum of solutions in the dual variable $\boldsymbol{\lambda}$.

First, let us introduce the reduced (or dual) version of Problem (35), which is derived by eliminating the velocity variable $\mathbf{u}$

$$
\begin{aligned}
W \boldsymbol{\lambda}+\mathbf{w} & =\gamma \quad \boldsymbol{\lambda}, \boldsymbol{\gamma} \in \mathbb{R}^{2 \mathfrak{s}(d) n_{Q}} \\
f_{\mathrm{DS}}^{\star}(\boldsymbol{\lambda}, \gamma) & =\mathbf{0},
\end{aligned}
$$

where $W=R^{\dagger, \top} B A^{-1} B^{\top} R^{\dagger}$ and $\mathbf{w}=R^{\dagger, \top}\left(B A^{-1} \mathbf{l}+\mathbf{k}\right)$.

For any value of a parameter $s \in \mathbb{R}_{+}^{n_{Q}}$, we define the quadratic function

$$
J_{s}(\boldsymbol{\lambda}):=\frac{1}{2} \boldsymbol{\lambda}^{\top} W \boldsymbol{\lambda}+\boldsymbol{\lambda}^{T}(\mathbf{w}+(s ; 0))
$$

and the SOCQP

$$
\min _{\boldsymbol{\lambda} \in \mathcal{K}_{\mu}} J_{s}(\boldsymbol{\lambda})
$$

Corresponding optimality conditions read $\mathcal{K}_{\frac{1}{\mu}} \ni \nabla J_{s}(\boldsymbol{\lambda}) \perp$ $\lambda \in \mathcal{K}_{\mu}$, i.e.,

$$
\mathcal{K}_{\frac{1}{\mu}} \ni \gamma+(s ; 0) \perp \boldsymbol{\lambda} \in \mathcal{K}_{\mu}
$$

Let us introduce the mappings $v: \mathbb{R}_{+}^{n_{Q}} \rightarrow \mathbb{R}^{\mathfrak{s}(d) n_{Q}}, s \mapsto$ $v(s):=\nabla J_{s}\left(\boldsymbol{\lambda}^{*}(s)\right)$ with $\boldsymbol{\lambda}^{*}(s)$ a solution to the SOCQP (37), and $F: \mathbb{R}_{+}^{n_{Q}} \rightarrow \mathbb{R}_{+}^{n_{Q}}, s \mapsto \mu\left\|v(s)_{\mathrm{T}}\right\|$. Using a duality argument, Cadoux et al. [Cadoux, 2009, 
Acary et al., 2011] show that $v$ and therefore $F$ are uniquely defined.

Solving our problem amounts to finding a fixed-point $s^{*}$ of $F$, thus setting $\boldsymbol{\lambda}=\boldsymbol{\lambda}^{*}\left(s^{*}\right)$. Indeed $s^{*}=F\left(s^{*}\right)=$ $\mu\left\|\boldsymbol{\gamma}_{\mathrm{T}}+\left(s^{*} ; 0\right)_{\mathrm{T}}\right\|=\mu\left\|\boldsymbol{\gamma}_{\mathrm{T}}\right\|$, and therefore the KKT conditions (38) yield

$$
\mathcal{K}_{\frac{1}{\mu}} \ni \gamma+\left(\mu\left\|\gamma_{\mathrm{T}}\right\| ; 0\right) \perp \boldsymbol{\lambda} \in \mathcal{K}_{\mu} .
$$

We identify $\hat{\gamma}:=\gamma+\left(\mu\left\|\gamma_{\mathrm{T}}\right\| ; 0\right)$ and get $f_{\mathrm{DS}}^{\star}(\boldsymbol{\lambda}, \boldsymbol{\gamma})=\mathbf{0}$.

Data: $\boldsymbol{\lambda}$ initial guess

Data: $\varepsilon>0$ tolerance

Result: $(\boldsymbol{\lambda} ; \boldsymbol{\gamma})$ satisfying $(36)$

Loop

$$
\begin{aligned}
& \gamma \leftarrow W \boldsymbol{\lambda}+\mathbf{w} ; \\
& \text { break if }\left|f_{\mathrm{AC}}(\boldsymbol{\lambda}, \gamma)\right|<\varepsilon ; \\
& s \leftarrow \mu\|\gamma\| ; \\
& \boldsymbol{\lambda} \leftarrow \text { solution of SOCQP (37) at } s ;
\end{aligned}
$$$$
\text { end }
$$

Algorithm 1: Fixed-point algorithm with AlartCurnier stopping criterion

$F$ is not guaranteed to be a contraction; in Section (4.4.5) we will present some sufficient conditions for the existence of a fixed point. In practice, we observe very good convergence of the fixed-point algorithm 1. More complex rules for iterating on $s$ have been investigated in [Cadoux, 2009] but have not been found to perform significantly better.

\subsubsection{Second Order Cone Programming}

The easiest way to tackle the SOCQP (37) is to leverage the efficiency of out-of-the-box interior-points solvers for Second Order Cone Programs (SOCP), of which it is a subclass.

The first step consists in removing the quadratic part of the objective function by introducing a variable $t \in \mathbb{R}$ subject to the constraint $t \geq \frac{1}{2} \boldsymbol{\lambda}^{\top} W \boldsymbol{\lambda}$. The new objective is $\hat{J}_{s}(t, \boldsymbol{\lambda})=t+\boldsymbol{\lambda}^{T}(\mathbf{w}+(s ; 0))$.

Given a square root $L$ of $A$, we have $A=L L^{\top}$ and $W=\left(L^{-1} B^{T} R^{\dagger}\right)^{\top} L^{-1} B^{\top} R^{\dagger}$. With an auxiliary variable $\mathbf{y} \in \mathbb{R}^{\mathfrak{v}}$ that satisfies $L \mathbf{y}=B^{\top} R^{\dagger} \boldsymbol{\lambda}$, the constraint on $t$ now reads $2 t \geq \mathbf{y}^{\top} \mathbf{y}$ and may also be written as a rotated cone constraint $(1, t, \mathbf{y}) \in \mathcal{R} \mathcal{K}$.

We obtain the SOCP

$$
\begin{aligned}
\min _{t \in R, \boldsymbol{\lambda} \in \mathbb{R}^{\mathfrak{s}(d)} n_{Q}} t+\boldsymbol{\lambda}^{\top}(\mathbf{w}+(s ; 0)) \\
R \mathbf{z}=\boldsymbol{\lambda} \\
L \mathbf{y}=B^{\top} \mathbf{z} \\
\boldsymbol{\lambda} \in \mathcal{K}_{\mu}^{n_{Q}} \\
(1, t, \mathbf{y}) \in \mathcal{K}_{1} .
\end{aligned}
$$

\subsubsection{Projected Gradient}

The interior-point approach has some drawbacks: arguably, the reliance on a complex external code, but most importantly, the inability of interior-point solvers to be warm-started. As our outer fixed-point loop converges, the quality of our initial guess increases and we would like the SOCQP solver to take advantage of this.

Another natural way to solve the SOCQP is to use an algorithm of the Projected-Gradient (or Projected Gradient Descent) families (PG). In its simplest form, such an algorithm reads:

Data: $\boldsymbol{\lambda}$ initial guess

Data: Step length $\xi>0$

Result: $\boldsymbol{\lambda}$ solution of SOCQP (37)

Loop

$$
\begin{aligned}
& \mathbf{y} \leftarrow W \boldsymbol{\lambda}+\mathbf{w}+(s ; 0) \\
& \mathbf{x} \leftarrow \Pi_{\mathcal{K}_{\mu}}(\boldsymbol{\lambda}-\xi \mathbf{y}) \\
& \text { break if }|\mathbf{x}-\boldsymbol{\lambda}|<\varepsilon \\
& \boldsymbol{\lambda} \leftarrow \mathbf{x}
\end{aligned}
$$

end

Algorithm 2: Projected Gradient Descent algorithm.

Since Algorithm (2) can converge quite slowly in practice, several works have focused on finding ways to improve its speed, among which we note

- Performing a line-search of the step size $\xi$;

- The Accelerated Gradient Descent method from Nesterov [Nesterov, 1983], adapted to SOCQP in [Heyn, 2013];

- The Spectral Gradient method from Barzilai et al. [Barzilai and Borwein, 1988], adapted to SOCQP in [Tasora, 2013].

Using such techniques, we found the PG method to be competitive with interior-points solvers on our problem, especially as the outer fixed-point loop converges and the quality of the initial guess increases. Numerical details are provided in Section 6.4.

\subsubsection{Primal algorithm}

In some situations, one may only be interested in the primal variable $\mathbf{u}$, and may not need the value of the dual variable $\boldsymbol{\lambda}$. In this case, following [Cadoux, 2009] again, we can implement the fixed-point algorithm directly on the primal formulation, and avoid the need for some auxiliary variables.

The insight is that the SOCQP (37) is the dual of another optimization problem, namely

$$
\min _{\mathbf{u} \in C(s)} J^{*}(\mathbf{u})
$$

with

$$
\begin{aligned}
J^{*}(\mathbf{u}) & :=\frac{1}{2} \mathbf{u}^{\top} A \mathbf{u}-\mathbf{u}^{\top} \mathbf{l} \\
C(s) & :=\left\{\mathbf{u} \in \mathbb{R}^{\mathfrak{v}}, R^{\dagger, \top}(B \mathbf{u}+\mathbf{k})+(s ; 0) \in \mathcal{K}_{\frac{1}{\mu}}\right\} .
\end{aligned}
$$

In contrast to the dual (37), the SOCQP (39) is strictly convex, and therefore guaranteed to admit a unique solution as long as the feasible set is not empty. 
Data: u initial guess

Data: $\varepsilon>0$ tolerance

Result: u satisfying (35)

Loop

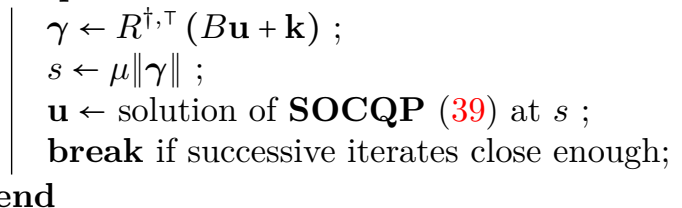

Algorithm 3: Primal version of the fixed-point algorithm

Algorithm 1 can then be trivially adapted to use the primal minimization problem, as in Algorithm 3. Due the to complex nature of the constraint, the SOCQP (39) is not suited for a Projected-Gradient algorithm, however it can be just as easily put under a SOCP form.

$$
\begin{aligned}
\min _{t \in R, \mathbf{u} \in \mathbb{R}^{\mathfrak{v}}} t & \mathbf{u}^{\top} \mathbf{l} \\
R^{\top} \mathbf{z} & =B \mathbf{u}+\mathbf{k}+R^{\top}(s ; 0) \\
\mathbf{y} & =L^{\top} \mathbf{u} \\
\mathbf{z} & \in \mathcal{K}_{\frac{1}{\mu}}^{n_{Q}} \\
(1, t, \mathbf{y}) & \in \mathcal{K}_{1}
\end{aligned}
$$

\subsubsection{Existence of solutions}

Criterions that ensure the existence of solutions to the discrete Coulomb friction problem, of which Problem (35) is an instance, are given in [Cadoux, 2009, Acary et al., 2011]; we restate them below. Note that those criterions are only sufficient, and not necessary. First, the hypothesis $\overline{\mathcal{H}}(\mu)$,

$$
\overline{\mathcal{H}}(\mu):=\exists \mathbf{u}, R^{\dagger, \top}(B \mathbf{u}+\mathbf{k}) \in \mathcal{K}_{\frac{1}{\mu}}
$$

ensures the existence of a fixed-point $s^{*}$ of $F$, i.e., such that $s^{*}=F\left(s^{*}\right)$. The velocity $\mathbf{u}$ is given by the unique solution to the primal problem (39) at $s^{*}$, and the strain rate tensor $\gamma$ can be deduced from $\mathbf{u}$. The existence of a corresponding stress field $\boldsymbol{\lambda}$ boils down to

$$
\exists \boldsymbol{\lambda} \in \mathcal{K}_{\mu}, \quad B^{\top} R^{\dagger} \boldsymbol{\lambda}=A \mathbf{u}-\mathbf{l} \text { and } \hat{\gamma}^{\top} \boldsymbol{\lambda}=0
$$

which is automatically satisfied under a stronger hypothesis,

$$
\mathcal{H}(\mu):=\exists \mathbf{u}, R^{\dagger, \top}(B \mathbf{u}+\mathbf{k}) \in \operatorname{Int} \mathcal{K}_{\frac{1}{\mu}} .
$$

Proof. $\mathcal{H}(\mu)$ yields directly that $\operatorname{Int} C(s) \neq \varnothing$. As $\mathbf{u}$ is a solution of $(39),-\nabla J^{*}(\mathbf{u}) \in \mathcal{N}_{C(s)}(\mathbf{u})$. We have

$$
\mathcal{N}_{C(s)}(\mathbf{u})=\partial \mathcal{I}_{C(s)}(\mathbf{u})=\partial \mathcal{I}_{\mathcal{K}_{\frac{1}{\mu}}}\left(R^{\dagger, \top}(B \mathbf{u}+\mathbf{k})+(s ; 0)\right)
$$

where $\mathcal{I}_{C}(\mathbf{x})$ denotes the value of the characteristic function of the convex set $C$ at $\mathbf{x}$. As $\operatorname{Int} C(s) \neq \varnothing$, the latter subdifferential yields exactly

$$
\mathcal{N}_{C(s)}(\mathbf{u})=B^{T} R^{\dagger} \partial \mathcal{I}_{\mathcal{K}_{\frac{1}{\mu}}}(\mathbf{u})=B^{\top} R^{\dagger} \mathcal{N}_{\mathcal{K}_{\frac{1}{\mu}}}(\mathbf{u})
$$

Finally, we have $\mathcal{N}_{\mathcal{K}_{\frac{1}{\mu}}}(\mathbf{u}) \subset-\mathcal{K}_{\mu} \cap\{\hat{\gamma}\}^{\perp}$, and therefore $A \mathbf{u}-\mathbf{l}=\nabla J^{*}(\mathbf{u}) \in B^{\top} R^{\dagger}\left(\mathcal{K}_{\mu} \cap\{\hat{\gamma}\}^{\perp}\right)$.

In the case $\mu=0$, the strong hypothesis $\mathcal{H}(\mu)$ amounts to the existence of a velocity field with strictly positive divergence everywhere - this requires outward Dirichlet boundary conditions, or a Neumann boundary. The weaker $\overline{\mathcal{H}}(\mu)$ only requires that there exist a velocity field with nowhere strictly negative divergence.

Homogeneous Dirichlet boundary conditions only satisfy the latter, weaker criterion $\overline{\mathcal{H}}(\mu)$. However we can always exhibit a trivial solution in $\mathbf{u}, \boldsymbol{\lambda}$; for $\mu=0$, it corresponds to the incompressible Stokes solution, and for $\mu>0$, to the null velocity solution, $\mathbf{u}=0$.

Proof. By construction we have $\mathbf{k}=0$. Moreover, the condition imposes $\int_{\Omega} \nabla \cdot \mathbf{u}=\int_{\partial \Omega} \mathbf{u}_{D} \cdot \mathbf{n}=0$. As the rheology requires $\nabla \cdot \mathbf{u} \geq 0$, velocity solutions must have null divergence, which means in the discrete case $\left(R^{\dagger, \top} B \mathbf{u}\right)_{\mathrm{N}}=0$.

In the case $\mu=0, s^{*}=0$ is a fixed-point of $F$, and the primal feasibility condition $\mathbf{u} \in C(0)$ boils down to $\left(R^{\dagger, \top} B \mathbf{u}\right)_{\mathrm{N}}=0$. The optimality condition reads

$$
-\nabla J^{*} \in \mathcal{N}_{C(0)}(\mathbf{u})=\left(\operatorname{Ker}\left(R^{\dagger, \top} B\right)_{\mathrm{N}}\right)^{\perp}=\operatorname{Im}\left(R^{\dagger, \top} B\right)_{\mathrm{N}}^{\top},
$$

so there exists $\boldsymbol{\lambda}$ with $\nabla J^{*}=B^{T} R^{\dagger} \boldsymbol{\lambda}$ and $\boldsymbol{\lambda}_{\mathrm{T}}=0$.

In the case $\mu>0, \mathbf{u} \in C(0)$ boils down to $R^{\dagger, \top} B \mathbf{u}=0$. This means that the solution $\mathbf{u}^{*}(0)$ of the primal problem at $s=0$ satisfies $\left\|\left(B^{\top} R^{\dagger} \mathbf{u}\right)_{\mathrm{T}}\right\|=0$, and therefore $s^{*}=0$ is a fixed point of $F$. The optimality condition yields $\exists \boldsymbol{\lambda}, \nabla J^{\star}=B^{T} R^{\dagger} \boldsymbol{\lambda}$.

In both cases, we define $\boldsymbol{\lambda}^{*}:=\boldsymbol{\lambda}+\left(\Delta_{p} ; 0\right)$, with $\Delta_{p}$ constant over all discretization points. By construction, $\boldsymbol{\lambda}-\boldsymbol{\lambda}^{*} \in \operatorname{Ker} B^{T} R^{\dagger}$. We can choose $\Delta_{p}$ such that $\boldsymbol{\lambda}^{*} \in \mathcal{K}_{\mu}$; for $\mu=0$, it suffices to take $\Delta_{p}:=-\min \boldsymbol{\lambda}_{\mathrm{N}}$, and for $\mu>0$, $\Delta_{p}:=-\min \left(\boldsymbol{\lambda}_{\mathrm{N}}-\frac{1}{\mu} \boldsymbol{\lambda}_{\mathrm{T}}\right)$. We have then $A \mathbf{u}-\mathbf{l}=\nabla J^{*}=$ $B^{T} R^{\dagger} \boldsymbol{\lambda}^{*}$, and it can be easily verified that $\hat{\boldsymbol{\gamma}}^{\top} \boldsymbol{\lambda}^{*}=0$.

\subsection{Solving (35) numerically in the case $\mathrm{Bi}>0$}

The case $\mathrm{Bi}>0$, where the $\mathbf{S O C} \mathcal{K}_{\mu}$ is replaced with the truncated one $\mathcal{K}_{\mu, \mathrm{Bi}}$, has not been as extensively studied in the literature. In the following sections, we show that we can extend the framework presented above to $\mathrm{Bi}>$ 0 . Furthermore, a simple modification to the ProjectedGradient algorithm will be sufficient to solve our problem.

\subsubsection{Duality}

Theorem 4. We introduce a modification of the objective function of the primal problem (39),

$$
J_{\mathrm{Bi}}^{*}(\mathbf{u}):=J^{*}(\mathbf{u})+\mathrm{Bi} \sum_{i=1}^{n_{Q}}\left\|\left(R^{\dagger, \top}(B \mathbf{u}+\mathbf{k})\right)_{T}^{i}\right\|,
$$

and the two optimization problems,

$$
\begin{aligned}
& \min _{\mathbf{u} \in C(s)} J_{\mathrm{Bi}}^{\star}(\mathbf{u}) \\
& \min _{\boldsymbol{\lambda} \in \mathcal{K}_{\mu, \mathrm{Bi}}} J_{s}(\boldsymbol{\lambda}) .
\end{aligned}
$$


Then, under the hypothesis that $\operatorname{Int} C(s) \neq 0$, it holds that (42) and (43) are dual problems, in the sense of Fenchel. Moreover, the optimal solution $\mathbf{u}$ of (42) and any optimal solution $\boldsymbol{\lambda}$ of (43) satisfy

$$
\left\{\begin{aligned}
A \mathbf{u} & =B^{\top} R^{\dagger} \boldsymbol{\lambda}+\mathbf{l} \\
\hat{\gamma} & =R^{\dagger, \top} B \mathbf{u}+R^{\dagger, \top} \mathbf{k}+(s ; 0) \\
\boldsymbol{\lambda} & =\Pi_{\mathcal{K}_{\mu, \mathrm{Bi}}}(\boldsymbol{\lambda}-\xi \hat{\gamma}) .
\end{aligned}\right.
$$

The corresponding proof is given in A.4.

This motivates the use of a fixed-point algorithm similar to the one introduced in Section 4.4.1, as the existence and well-posedness results can be directly transposed to this new couple of dual problems. The new primal problem (42) can just as easily be cast into a SOCP, as the sum of Euclidean norms in the objective function can be transformed into conical constraints by introducing a set of $n_{Q}$ auxiliary variable $\left(s_{i}\right)$ satisfying $s_{i} \geq\left\|\left(R^{\dagger, \mathrm{T}}(B \mathbf{u}+\mathbf{k})\right)_{\mathrm{T}}^{i}\right\|$ (see e.g. [Bleyer et al., 2015]). Adapting the dual algorithm is even simpler, as a slight modification of the Projected-Gradient algorithm is sufficient.

\subsubsection{Modified PG algorithm}

We modify Algorithm 2 so that $\boldsymbol{\lambda}-\boldsymbol{\xi} \mathbf{y}$ is projected onto $\mathcal{K}_{\mu, \mathrm{Bi}}$ instead of $\mathcal{K}_{\mu}$. When the algorithm converges, we have $\Pi_{\mathcal{K}_{\mu, \mathrm{Bi}}}\left(\boldsymbol{\lambda}-\xi \nabla J_{s}(\boldsymbol{\lambda})\right)=0$, which means that $\boldsymbol{\lambda}$ is a solution of the minimization problem (43).

Now, suppose that $s^{*} \in \mathbb{R}_{+}^{n_{Q}}$ is a fixed-point of the Cadoux algorithm with the new applications defined as

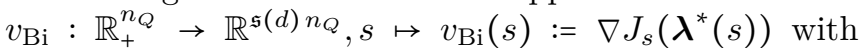
$\boldsymbol{\lambda}^{*}(s)$ a solution to $(43)$, and $F_{\mathrm{Bi}}: \mathbb{R}_{+}^{n_{Q}} \rightarrow \mathbb{R}_{+}^{n_{Q}}, s \mapsto$ $\mu\left\|v_{\mathrm{Bi}}(s)_{\mathrm{T}}\right\|$.

Then once again we have $v_{\mathrm{Bi}}\left(s^{*}\right)=\gamma+\left(\mu\left\|\boldsymbol{\gamma}_{\text {т }}\right\| ; 0\right)=\hat{\gamma}$, and therefore $\Pi_{\mathcal{K}_{\mu, \mathrm{Bi}}}(\boldsymbol{\lambda}-\xi \hat{\gamma})=0$ which exactly amounts to $f_{\mathrm{DS}}^{\star}(\boldsymbol{\lambda}, \gamma)=\mathbf{0}$.

\section{$5 \quad$ Inertial flows}

\subsection{Continuous equations}

We now add an inertial term to our momentum conservation equations. We only consider purely compressive scenarios, so the density $\rho$ is assumed to remain constant.

$$
\rho\left(\frac{\partial \mathbf{u}}{\partial t}+(\mathbf{u} \cdot \nabla) \mathbf{u}\right)-\nabla \cdot\left[2 \nu \mathrm{D}^{b}(\mathbf{u})+\boldsymbol{\tau}\right]+\nabla p=\rho \mathbf{g}
$$

With the dimensionless time $\tilde{t}$ defined such that $t=$ $\frac{L}{U} \tilde{t}=\sqrt{\frac{L}{g}} \tilde{t}$, the full system reads

$$
\begin{aligned}
\frac{\partial \tilde{\mathbf{u}}}{\partial \tilde{t}}+(\tilde{\mathbf{u}} \cdot \nabla) \tilde{\mathbf{u}}-\tilde{\nabla} \cdot\left[\frac{2}{\operatorname{Re}} \widetilde{\mathrm{D}^{b}}(\tilde{\mathbf{u}})-\tilde{\boldsymbol{\lambda}}\right] & =\mathbf{e}_{g} & & \text { on } \Omega \\
\widetilde{\mathbf{u}} & =\tilde{\mathbf{u}}_{D} & & \text { on } B_{D} \\
\widetilde{\mathrm{D}^{b}}(\tilde{\mathbf{u}}) \mathbf{n}_{\Omega} & =0 & & \text { on } B_{N} \\
(\tilde{\boldsymbol{\lambda}} ; \tilde{\mathrm{D}}(\tilde{\mathbf{u}})) \in \mathcal{D} \mathcal{P}(\mu, \mathrm{Bi}) & & & \text { on } \Omega
\end{aligned}
$$

As in the previous section, we will drop the tildes from now on.

\subsection{Discretization}

The notation $\frac{\partial \mathbf{u}}{\partial t}$ in previous paragraphs is misused. Indeed, due to the nonsmoothness of the rheology $\mathcal{D P}(\mu, \mathrm{Bi}), \mathbf{u}(t)$ may not be time-differentiable at every instant. Rigorously, it should be written as a measure differential inclusion. Fortunately, the so-called Moreau sweeping process [Moreau, 1999] ensures that a standard timestepping (or catch-up) algorithm will still converge as expected towards the continuous solution as the timestep $\Delta_{t}$ is refined.

While directly discretizing directly the transport term $(\mathbf{u} \cdot \nabla) \mathbf{u}$ would be tempting, this ultimately leads to the addition of a non-symmetric form to our equations, which breaks our resolution framework.

Instead, we use a more classical first-order characteristics scheme. The total derivative is approximated as

$$
\frac{\partial \mathbf{u}}{\partial t}+(\mathbf{u} \cdot \nabla) \mathbf{u} \sim \frac{\mathbf{u}^{k+1}-\mathbf{u}^{k} \circ X^{k}}{\Delta_{t}}
$$

where $X^{k}(x):=x-\Delta_{t} \mathbf{u}^{k}(x)$

The variational formulation is obtained by taking the forms defined in paragraph 4.2 and adding the following terms to $l$ and $a$,

$$
\begin{aligned}
a(\mathbf{u}, \mathbf{v}) & :=a_{(4.2)}(\mathbf{u}, \mathbf{v})+\frac{1}{\Delta_{t}} \int_{\Omega} \mathbf{u} \cdot \mathbf{v} \\
l(\mathbf{v}) & :=l_{(4.2)}(\mathbf{v})+\frac{1}{\Delta_{t}} \int_{\Omega}\left(\mathbf{u}^{k} \circ X^{k}\right) \cdot \mathbf{v} .
\end{aligned}
$$

Solving for each timestep in the dynamics setting is therefore equivalent to solving the problem defined in Section 4.

Remark 2. Note that the time-stepping scheme ensures a positive-definite form a even when $\frac{1}{\mathrm{Re}}=0$. This allows us to simulate purely plastic flows, instead of being restricted to viscoplastic ones as in Section 4.

\section{Results}

All the finite element simulations presented in this section were performed using the open-source library Rheolef [Saramito, 2012]. When solving the SOCP formulation of the inner problem, we used the commercial package MOSEK [Andersen et al., 2003]. The benchmarks were run on a standard quad-core Intel(R) Xeon W3520 machine with 8GB memory.

\subsection{Model problems}

In this section, we validate our solver on simple problems for which we can derive analytic solutions. 


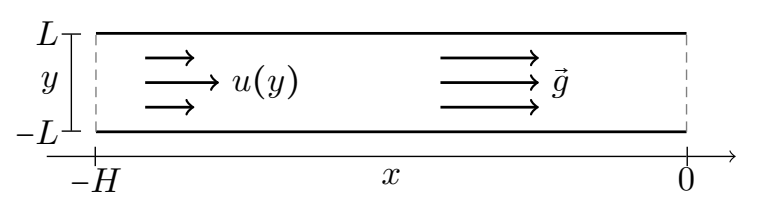

Figure 3: 2D channel for the Bingham Poiseuille flow
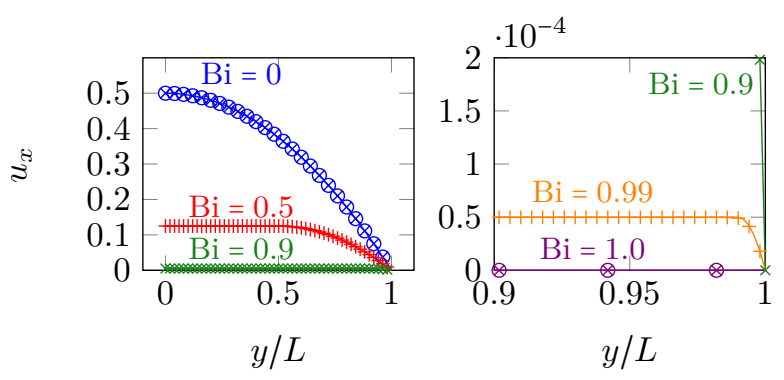

Figure 4: Comparison of the steady velocity profiles $u_{x}(-H / 2, y)$ between our numerical model using a $\mathbb{P}_{2}-$ $\mathbb{P}_{1 d}$ approximation (marks), and the analytic Bingham Poiseuille flow (lines). Plots for high values of $\mathrm{Bi}$ (right) are zoomed in compared to plots for lower Bi values (left).

\subsubsection{Bingham Poiseuille flow}

We consider a Bingham Poiseuille flow as illustrated in Figure 3, with the following boundary conditions,

$$
\begin{array}{r}
\mathbf{u}(x,-L)=\mathbf{u}(x, L)=0 \quad \forall x \in[-H, 0] \\
\mathbf{u}(-H, y)=u_{x}(-H, y) \mathbf{e}_{x} \forall y \in[-L, L] \\
\mathbf{u}(0, y)=u_{x}(0, y) \mathbf{e}_{x} \forall y \in[-L, L] .
\end{array}
$$

Figure 4 shows velocity profiles for different values of $\mathrm{Bi}$, for both the analytic solution and our model using a $\mathbb{P}_{4}-\mathbb{P}_{1 d}$ approximation. Using a nonsmooth solver allows us to recover the correct profile even for high values of $\mathrm{Bi}$ without any parameter tuning.

\subsubsection{Convergence of spatial discretization}

We study how the error between our method and the analytic solutions for $\mathrm{Bi}=0.5$ and $\mathrm{Bi}=0.9$ decreases as we uniformly refine a mesh with initial characteristic edge length $h_{0}$. Results for various FEM approximation orders are shown in Figure 5. Convergence was observed for all approximation orders, and we found that $\mathbb{P}_{2}-\mathbb{P}_{1}$ and $\mathbb{P}_{2}-\mathbb{P}_{1 d}$ achieved a good ratio of convergence speed versus computational cost. Conversely, the higher-order approximation $\mathbb{P}_{3}-\mathbb{P}_{2 d}$ performed relatively poorly on the finer meshes, which we interpret as being the result of numerically more complex quadrature rules. $\mathbb{P}_{4}-\mathbb{P}_{1 d}$, with high-order velocities but low-order stresses, yielded consistently the best results, at the cost of a very large $A$ matrix.

\subsubsection{Bagnold profiles}

We consider the flow of a granular layer of height $H=1$ on a rough infinite inclined plane with angle $\alpha$, as described
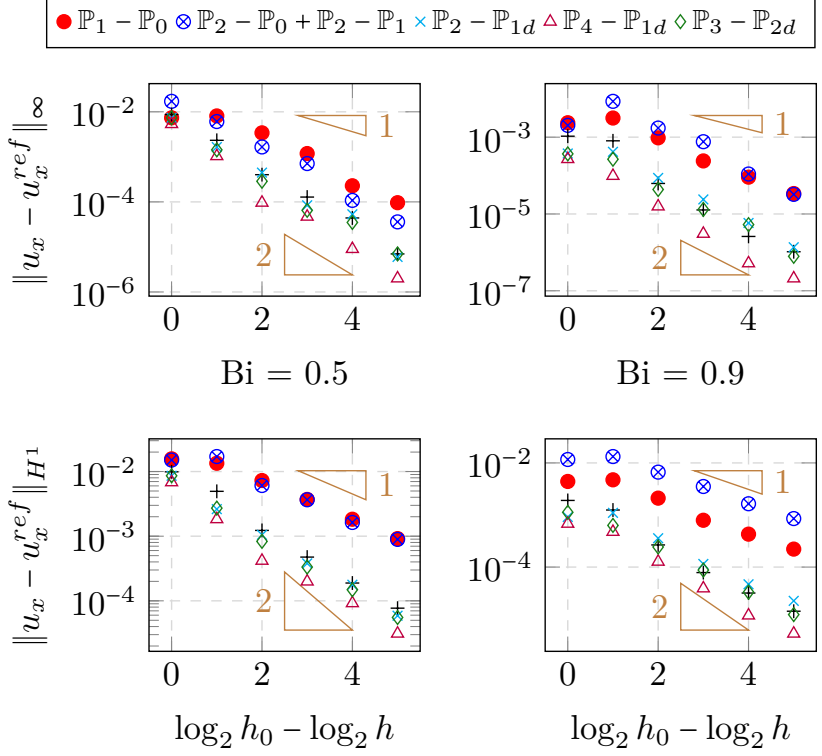

Figure 5: $L_{\infty}$-norm (top) and $H^{1}$-norm (bottom) convergence plots for various FEM approximation orders, for $B i=0.5$ (left) and $B i=0.9$ (right). $u_{x}^{r e f}$ denotes the analytic solution.

in [Lagrée et al., 2011] and illustrated in Figure 6. We assume the flow to be slow enough to neglect inertial terms.

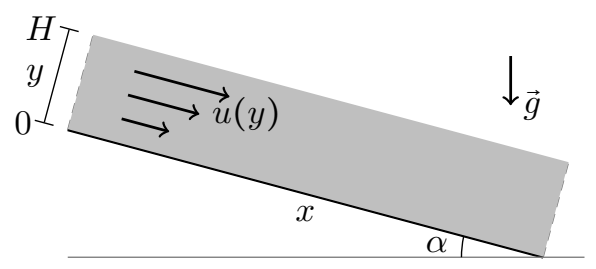

Figure 6: Flow on an infinite inclined plane

The conservation of momentum on the longitudinal $x$ axis and perpendicular $y$-axis reads

$$
\begin{aligned}
\frac{\partial}{\partial y}\left(\eta \frac{\partial u}{\partial y}+\tau_{x y}\right) & =-\sin \alpha \\
\frac{\partial p}{\partial y} & =-\cos \alpha .
\end{aligned}
$$

Integrating the second equation with the condition that the pressure should be zero at the top of the granular layer, i.e., $p(H)=0$, gives $p=(1-y) \cos \alpha$.

We consider the case of an avalanching flow with $\frac{\partial u}{\partial y}>$ 0 for $y<1$. This means that the friction is saturated, therefore $\tau_{x y}=\mu(1-y) \cos (\alpha)$. We get

$$
\frac{\partial}{\partial y} \eta \frac{\partial u}{\partial y}=-\sin \alpha+\mu \cos \alpha
$$

A Neumann boundary condition at the interface $\frac{\partial u}{\partial y}(1)=0$ imposes $\frac{\partial}{\partial y} \eta \frac{\partial u}{\partial y}<0$, which means $\mu<\tan \alpha$.

For spatially constant (Newtonian) $\eta$ and $\mu$, we get $\frac{\partial u}{\partial y}=\frac{1-y}{\eta} \sin \alpha-(\mu \cos \alpha)$, and $u(y)$ is quadratic. In order 
to retrieve the typical $\frac{3}{2}$ power of the Bagnold profile, we can instead choose $\eta(y):=|\mathrm{D}(u)|=\frac{1}{2} \frac{\partial u}{\partial y}$, which gives the analytic expressions

$$
\begin{aligned}
\frac{\partial u}{\partial y} & =\sqrt{2 \sin \alpha-(\mu \cos \alpha)}(1-y)^{\frac{1}{2}} \\
u(y) & =\frac{2}{3} \sqrt{2 \sin \alpha-(\mu \cos \alpha)}\left(1-(1-y)^{\frac{3}{2}}\right) .
\end{aligned}
$$

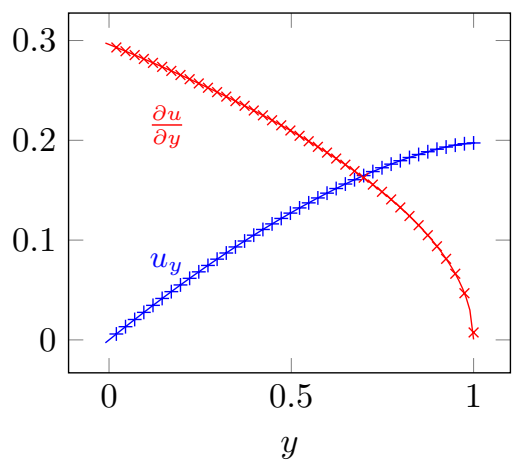

Figure 7: Comparison between our numerical solution (marks) and the analytic one (lines), for the velocity and shear-rate profiles of a Bagnold avalanche flow with $\mu=$ 0.5 and $\alpha=\tan ^{-1}(1.1 \mu)$.

We simulated this model on a square patch using the algorithm presented in Section 4. The value of $\boldsymbol{\sigma}_{\text {ext }}$ on the upstream and downstream boundaries was computed using the analytic solution. In order to handle the nonconstant $\eta$, we used a fixed-point algorithm which happened to converge very fast in practice - a dozen or so of iterations.

Numerical and analytic profiles are compared in Figure 7 . Once again, we observe a very good agreement between our model and the analytic solution.

\subsection{Discharge of a silo}

In this section we attempt to simulate a problem that has been widely studied both experimentally and numerically: the discharge of a granular silo.

\subsubsection{Beverloo scaling}

One of the most widely accepted macroscopic feature of the granular flow in a silo is the so-called Berveloo scaling [Beverloo et al., 1961], stating that the discharge rate $Q$ depends on the diameter of the outlet to the power $d-\frac{1}{2}$,

$$
Q=C \sqrt{g}\left(L-k d_{p}\right)^{d-\frac{1}{2}}
$$

where $d_{p}$ is the diameter of a grain, and $C$ and $k$ are dimensionless constants depending on the silo geometry and granular properties. The number $k$ typically lies within the range $1<k<3$.

The Beverloo phenomenon is particularly relevant for us as it has been shown that such a scaling cannot be recovered for Newtonian flows [Staron et al., 2012], nor for flows with a yield stress that does not depend on the pressure. The physical justification of the scaling involves inertia [Mankoc et al., 2007], so it is hopeless to attempt to retrieve it solely with the formulation of Section 4. For this problem, we therefore use the dynamic setting presented in Section 5 .

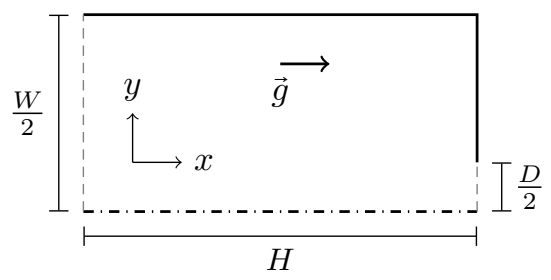

Figure 8: Geometry of a half-silo of height $H$, width $W$ and aperture size $D$

We simulated the 2D silo shown in Figure 8, with $W=$ $H=8 L$ and an aperture size $D$. We made the ratio $\tilde{D}:=\frac{D}{L}$ vary from $\frac{1}{2}$ to 2 , and studied the change in the dimensionless discharge rate $\tilde{Q}$ defined such that $Q$ := $\sqrt{g} L^{\frac{3}{2}} \tilde{Q}$.

Plots for different rheologies, with the corresponding Beverloo fits when one was found, are shown in Figure 9. Beverloo law coefficients for $\mu=0.6$, for which the fit was always acceptable except for $\mathrm{Re}=1$, are given in Table 1 . Coefficients for $\mu=0.4$ are also given when the fit was deemed of sufficient quality. We remark that decreasing Re, increasing $\mu$ or increasing $\mathrm{Bi}$ all contribute to a consequent increase of $k$. $\mu$ and $\mathrm{Bi}$ have also a positive impact on $C$, while Re has only a small influence on this parameter.

\begin{tabular}{cc|cc|cc} 
& & \multicolumn{2}{|c}{$\mathrm{Re}=100$} & $\mathrm{Re}=1000$ & $\mathrm{Re}=10$ \\
& & $\mathrm{Bi}=0$ & $\mathrm{Bi}=0.1$ & \multicolumn{2}{c}{$\mathrm{Bi}=0$} \\
\hline$\mu=0.3$ & $C$ & 1.70 & 1.44 & 1.77 & 2.00 \\
& $k$ & 0 & 0.96 & 0 & 3.34 \\
$\mu=0.4$ & $C$ & 1.54 & 1.52 & 1.54 & 1.48 \\
& $k$ & 0.87 & 2.49 & 0.38 & 3.07 \\
$\mu=0.5$ & $C$ & 1.35 & 1.43 & 1.35 & 1.33 \\
& $k$ & 0.83 & 3.2 & 0.22 & 3.56
\end{tabular}

Table 1: Beverloo law coefficents obtained for $\mu=0.6$ and $\mu=0.4$, for different $\mathrm{Bi}$ and Re values. The values for $k$ are given assuming $L=11.2 d_{p}$.

\subsubsection{Extension to the $\mu(I)$ rheology}

The $\mu(I)$ rheology is classically integrated into dynamics solvers by explicitly evaluating the value of the equivalent viscosity $\eta_{t o t}$ at each timestep [Lagrée et al., 2011].

In our case, we only have to explicitly evaluate the friction coefficient $\mu(I)$ instead of $\eta_{t o t}$. Since $\mu(I)$ can only take values in $\left[\mu_{s}, \mu_{s}+\Delta_{\mu}\right]$ whereas $\eta_{\text {tot }}$ takes values in $\mathbb{R}^{+}$, our approach has a few benefits:

- We do not have to clamp the value of $\eta_{t o t}$, and can 
- $\mu=0 \square \mu=0.1 \Delta \mu=0.2$

$\times \mu=0.3+\mu=0.4 \diamond \mu=0.5$

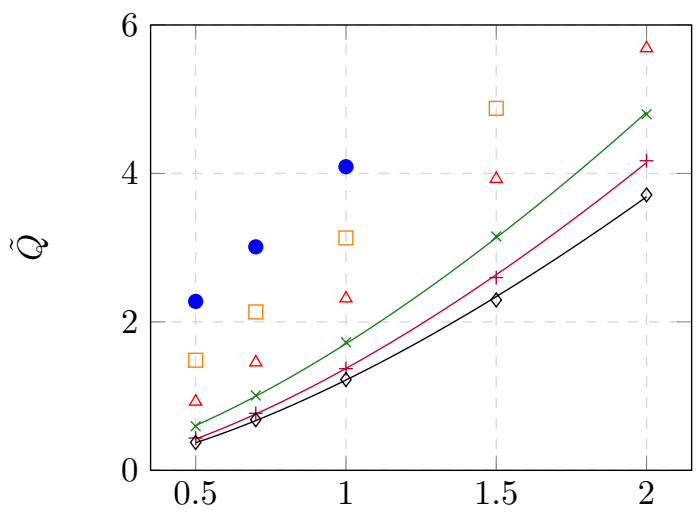

(a) $\operatorname{Re}=100, \mathrm{Bi}=0$

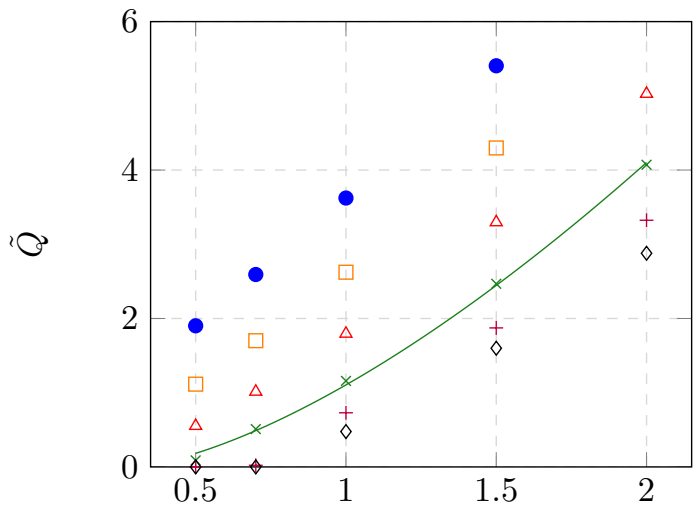

(c) $\mathrm{Re}=100, \mathrm{Bi}=0.2$

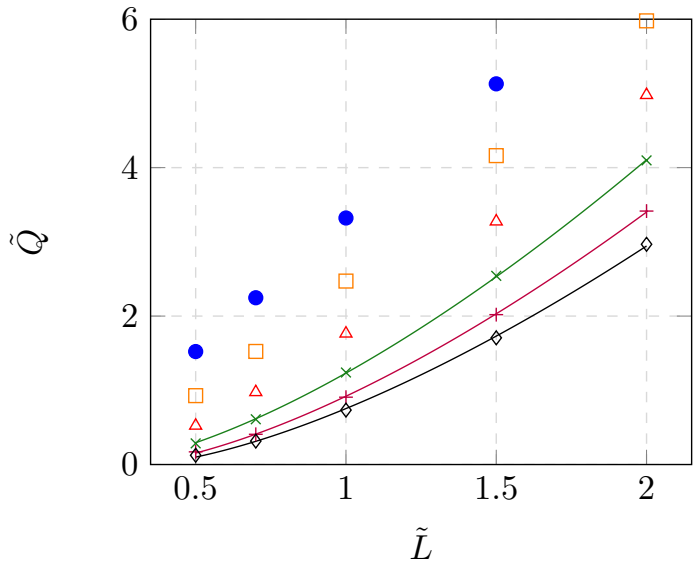

(e) $\operatorname{Re}=10, \mathrm{Bi}=0$
- $\mu=0 \square \mu=0.2$

$\triangle \mu=0.4 \times \mu=0.6$

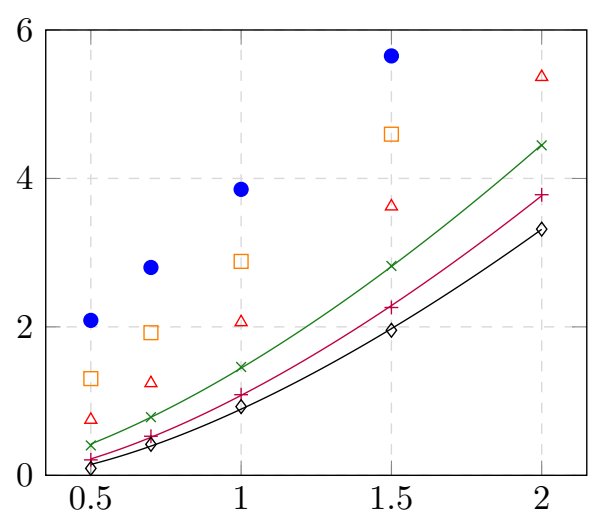

(b) $\mathrm{Re}=100, \mathrm{Bi}=0.1$

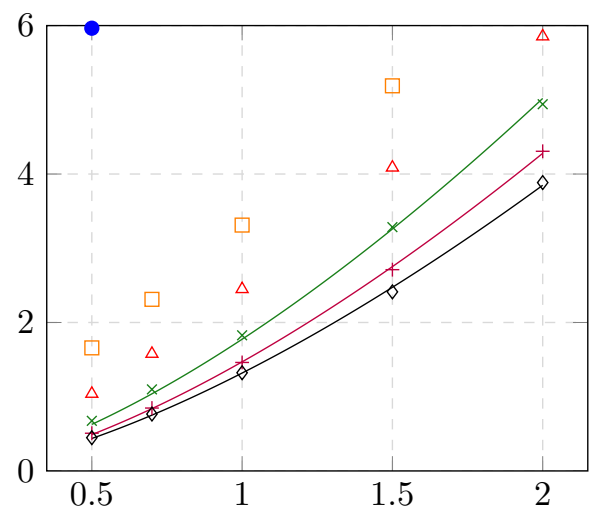

(d) $\mathrm{Re}=1000, \mathrm{Bi}=0$

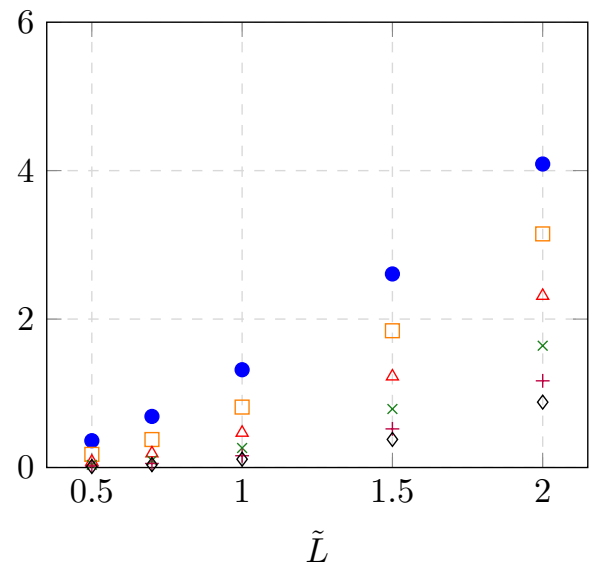

(f) $\mathrm{Re}=1, \mathrm{Bi}=0$

Figure 9: Dimensionless discharge rate $\tilde{Q}$ versus silo outlet diameter $\tilde{L}$ for and different rheologies. When $\mu$ is small, we cannot get a reasonable fit; in the case of the Newtonian flow, the discharge rate is closer to a linear law. Bi mainly influences the $k$ parameter of the law but does suffices to obtain a fit. Large values of Re tend to linearize the discharge rate for small $\mu$, smaller values make it quadratic. 
have fully rigid zones where the shear rate is strictly zero (which means an infinite $\eta_{t o t}$ )

- The loss of stability of the time-integration scheme due to this explicit term is much less dramatic

While the simulation frameworks are quite different - we do not take into account the air phase and use a non-zero $(\mathrm{Re}=100)$ Newtonian viscosity, we nevertheless attempted to recreate a simulation from [Staron et al., 2014, Fig 4], using the same physical parameters $\left(D=11.2 d_{p}, I_{0}=0.4, \mu=0.32, \Delta_{\mu}=0.28\right)$.

The results are shown in Figures 10 and 11; the match on the $C$ coefficient of the Beverloo law is surprisingly good (both methods give $C=1.48$ ), however we retrieve a coefficient $k$ that is significantly smaller ( 0.52 vs 0.73 ). Velocity profiles along the vertical and horizontal sections described in [Staron et al., 2014] are also presented in Figure 11.

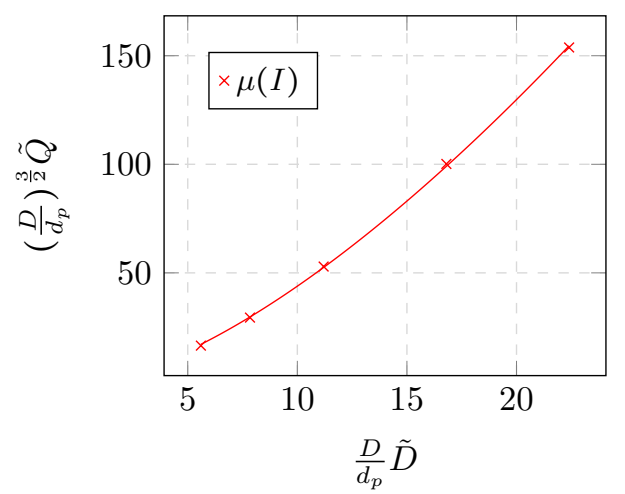

Figure 10: Discharge rate as a function of the outlet size for the $\mu(I)$ rheology.

Visualization of the velocity and stress fields Plots of the steady-state velocity and stress fields for flows with and without inertia are shown in B.

\subsection{Flow around a cylinder}

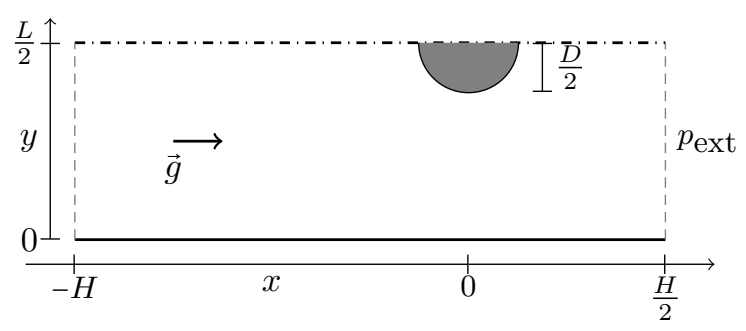

Figure 12: Flow around a cylinder of diameter $D$ in $2 \mathrm{D}$ channel of width $L$. The speed of the flow can be adjusted by varying the external pressure at the downstream boundary $p_{\text {ext }}$.

In this section, we study a gravity induced flow in a $2 \mathrm{D}$ channel of width $L$ around a cylinder of diameter $D$, as shown in Figure 12. We use no-slip boundary conditions for the velocity on the sides of the channel and on the cylinder, and homogeneous Neumann conditions at the upstream and downstream boundaries.

\subsubsection{Drag}

We attempt to reproduce the experimental setting presented in [Chehata et al., 2003]. Using a regularization of the $\mu(I)$ rheology, Chauchat and Médale [Chauchat and Médale, 2014] performed simulations that showed a good qualitative agreement with the experimental results. However, their approach suffered from two drawbacks:

- The regularization induces a creeping flow even when none should occur

- The $\nabla \cdot \mathbf{u}=0$ condition leads to negative pressures behind the obstacle. This is outside the domain of validity of the $\mu(I)$ rheology.

Our method also suffer from approximations; we assume a constant volume fraction of grains, even if this should clearly not be the case behind the obstacle. As the flow is slow, we also neglect its inertia. We use a constant friction coefficient, $\mu=0.5$, and set $\operatorname{Re}=100$ and $\mathrm{Bi}=0$. We vary the average upstream velocity $U_{\infty}$ by setting different values for the external pressure $p_{\text {ext }}$ at the downstream interface; the lower this pressure, the faster the grains will flow. This emulates the varying outlet size in [Chehata et al., 2003]. We set no external pressure at the upstream boundary.

The grains exercise a longitudinal drag force on the cylinder $O$ that can be computed as $F_{D}:=\int_{\partial O} \lambda \mathbf{n}_{O} \cdot \mathbf{e}_{x}$. The Froude number is defined from the adimensional average upstream velocity $U_{\infty}$ and the length ratio $\tilde{D}:=\frac{D}{L}$ as $\operatorname{Fr}:=\frac{U_{\infty}}{\sqrt{\tilde{D}}}$.

The dimensionless equivalent drag coefficient $C_{D}$ is then deduced as

$$
C_{D}:=\frac{F_{D}}{\frac{1}{2} U_{\infty}^{2} \tilde{D}}
$$

Figure 13 shows on a logarithmic scale the evolution of the drag coefficient $C_{D}$ with the Froude number (left) or average velocity (right), for different cylinder diameters. We retrieve the linear profiles observed in [Chehata et al., 2003, Chauchat and Médale, 2014]. The fact that the data points for the different diameters become aligned on the right plot means that the slope of $\frac{F_{D}}{\frac{1}{2} \tilde{D}}$ is independent of $\tilde{D}$; the drag force depends linearly on the diameter of the obstacle.

\subsubsection{Visualization of velocity and stress fields}

We consider a narrow channel $(L=4 D)$ with no-slip walls. Figure 14 collects plots of the velocity and stress fields across the domain. The pressure field is of special interest as it features a few notable phenomenons:

- First, it validates the benefit of allowing the dilation of the flow, as the pressure in the wake of the obstacle is indeed zero; 

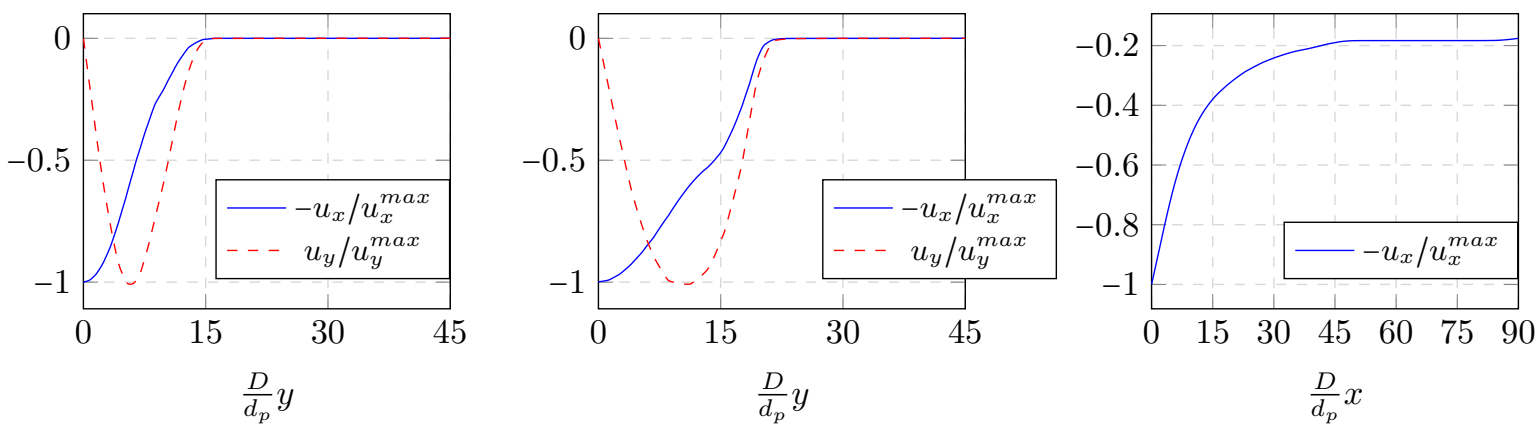

Figure 11: Horizontal and vertical velocity profiles for $\tilde{D}=1$ along section $S 1, S 2$ and $H 1$, as defined in [Staron et al., 2014, Fig. 6]
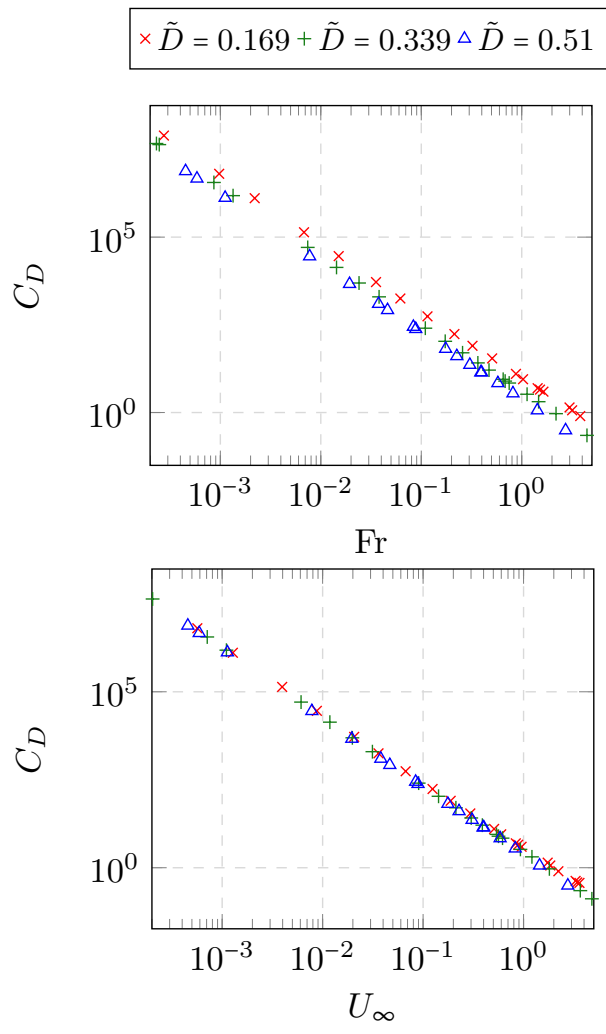

Figure 13: Drag coefficient $C_{D}$ versus Froude number Fr (left) and upstream velocity $U_{\infty}$ (right) for the granular flow around a cylinder

- The zone of highest pressure is not located at the very front of the obstacle. Instead, we observe the formation of high-pressure arch above this point;

- Above this arch, there exists a region where the pressure is lower than further upstream; moreover the transition between this two zone is very abrupt, as shown by the absence of two isosurfaces on the figure. Note that this phenomenon disappears when considering free-slip boundary conditions for the channel walls.

\subsection{Performance}

We now study the computational performance for the problem of Section 4 - the dynamics case is just a sequence of such problems.

In order to make as fair a comparison as possible, to evaluate the current error at each fixed-point iteration we chose not to use a residual based on a projection operator — which are known to be disadvantageous for interior-points - but rather the SOC FischerBurmeister function for frictional problems as defined e.g. in [Daviet et al., 2011].

We consider the silo geometry of Figure 8, a $\mathbb{P}_{2}-\mathbb{P}_{1 d}$ FEM approximation order, and a rheology with $\mu=0.5$, $\mathrm{Re}=1$ and $\mathrm{Bi}=0$. The base mesh has a resolution of 640 vertices.

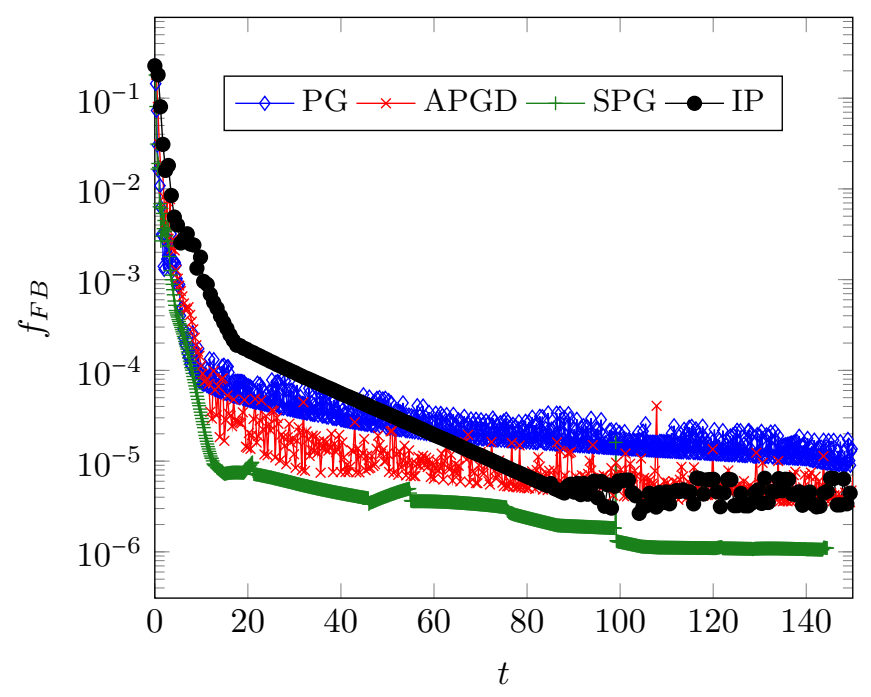

Figure 15: Infinity-norm of the Fischer-Burmeister function as a function of the wall time for the ProjectedGradient, Accelerated Projected Gradient Descent, Spectral Projected Gradient and Interior Points algorithm

Figure 15 shows typical convergence plots for selected algorithms. Table 2 shows the evolution of the computation time in seconds as the mesh is refined, for an interiorpoint method and a Spectral Projected Gradient method. The latter, despite its simplicity, is therefore highly competitive. 


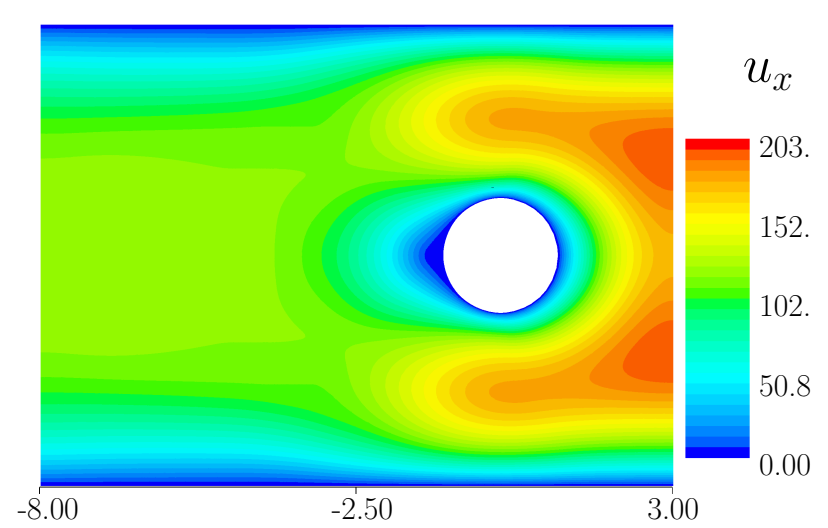

(a) Axial velocity $u_{x}$

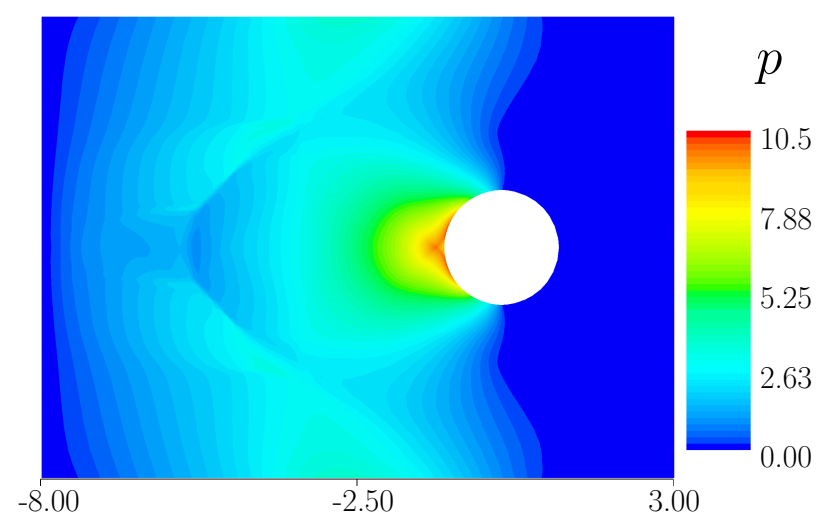

(c) Pressure $p$

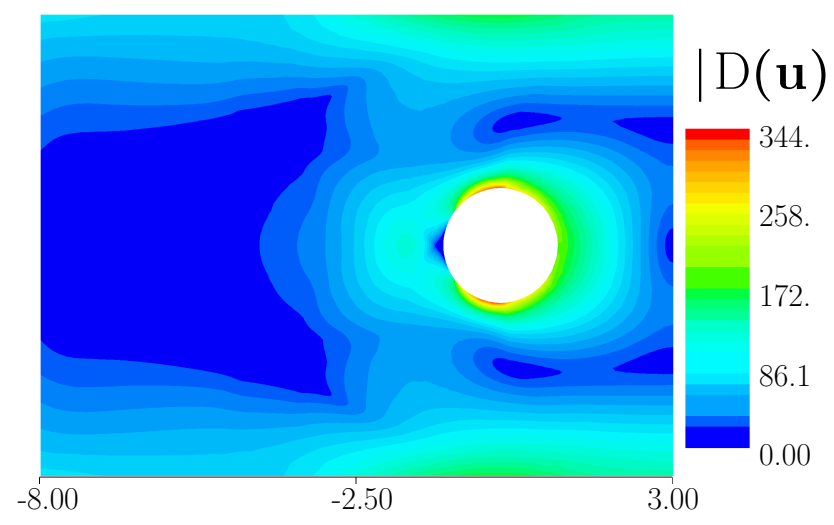

(e) Shear rate $|\mathrm{D}(\mathbf{u})|$

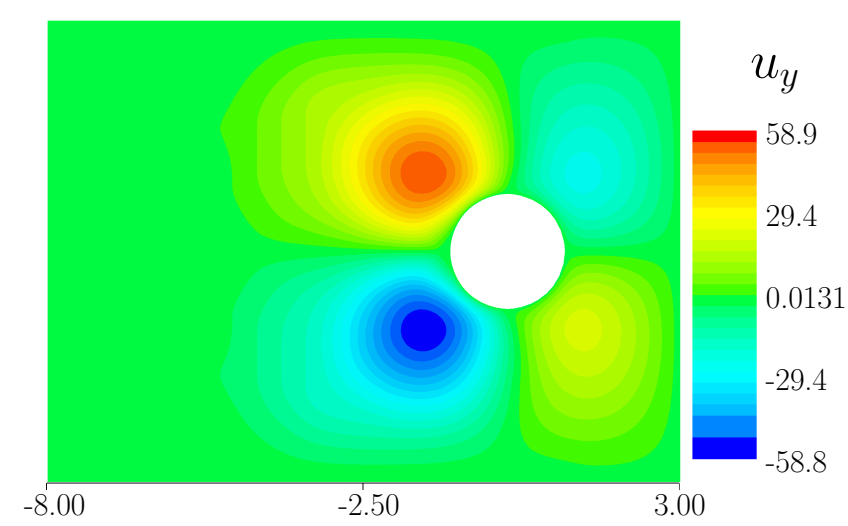

(b) Perpendicular velocity $u_{y}$

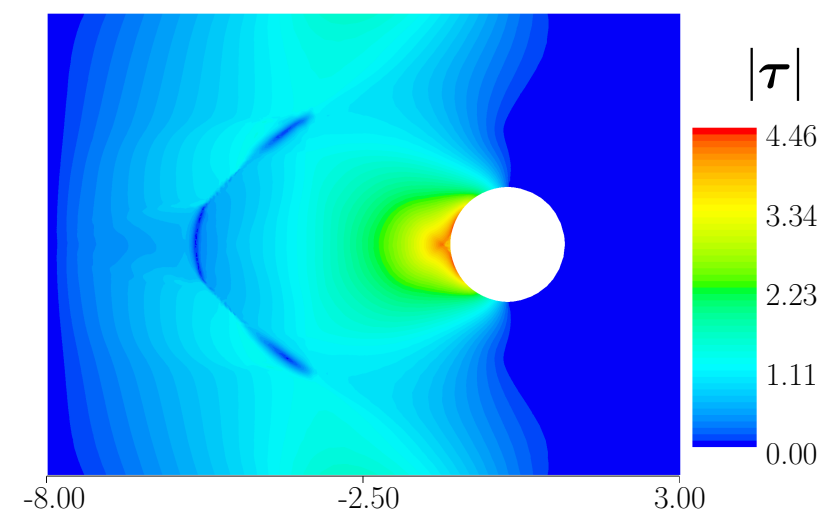

(d) Norm of frictional stress $|\boldsymbol{\tau}|$

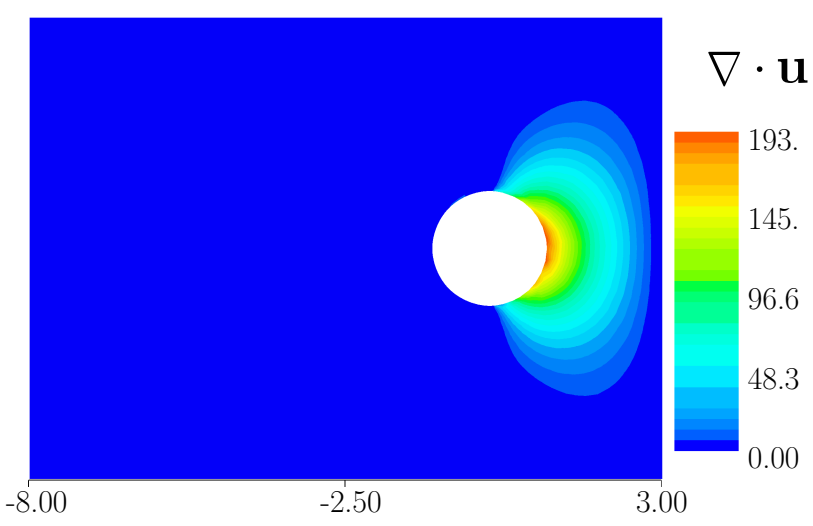

(f) Divergence of velocity $\nabla \cdot \mathbf{u}$

Figure 14: Velocity and stress fields for the flow around a cylinder in a narrow channel, with $\mu=0.3$, Re $=100$, $\mathrm{Bi}=0$ and $\tilde{D}=\frac{1}{4}$

\begin{tabular}{r|ccc|ccc|ccc|ccc} 
& \multicolumn{3}{|c}{ Mesh 1 } & \multicolumn{3}{c}{ Mesh 2 } & \multicolumn{3}{c}{ Mesh 3 } & \multicolumn{3}{c}{ Mesh 4} \\
Mosek & $\mathrm{t}$ & $\mathrm{n}$ & $\mathrm{t} / \mathrm{n}$ & $\mathrm{t}$ & $\mathrm{n}$ & $\mathrm{t} / \mathrm{n}$ & $\mathrm{t}$ & $\mathrm{n}$ & $\mathrm{t} / \mathrm{n}$ & $\mathrm{t}$ & $\mathrm{n}$ & $\mathrm{t} / \mathrm{n}$ \\
SPG & 11 & 100 & 0.71 & 116 & 29 & 4 & 564 & 23 & 24 & 6281 & 17 & 369 \\
& 88 & 0.14 & 69 & 79 & 0.88 & 144 & 33 & 4.4 & 1098 & 47 & 23
\end{tabular}

Table 2: Comparison of time taken to reach a give tolerance for 4 meshes with respectively 670, 2703, 10403 and 41509 vertices. The columns headers $\mathrm{t}, \mathrm{n}$, and $\mathrm{t} / \mathrm{n}$ give the total wall time in seconds, the number of iterations of the fixed-point algorithm and the average time per iteration. 


\section{Discussion}

\subsection{Limitations}

The main limitation of our approach appears to be its computational cost. The SOCP formulation becomes quickly expensive when the number of required fixedpoint iterations increases, while the Projected Gradient requires solving at least one linear system at each inner iteration. This makes three-dimensional scenarios only tractable for rough meshes. However, note that for the timestepping algorithm without Newtonian viscosity $(\operatorname{Re}=+\infty)$, the inverse of the $A$ matrix will be sparse for certain discretizations of $V(\Omega)$. The explicit computation of the Delassus operator would then become possible. This could speed up the proposed method, or allow for the use of other algorithms such as Gauss-Seidel based ones [Jourdan et al., 1998, Daviet et al., 2011].

From a physics point of view, the fact that we do not take into account a variable density leads to invalid predictions in zones where the local divergence is strictly positive, such as regions in the wake of obstacles. Our simple unilateral compressibility condition $\nabla \cdot \mathbf{u} \geq 0$ also prevents the material from recompacting after dilatation; a condition based on the current volume fraction, such as used in [Dunatunga and Kamrin, 2015], would be necessary to ensure the applicability of our method to general scenarios.

Our treatment of boundary conditions is also limited. As argued in [Domnik and Pudasaini, 2012], we should consider more realistic laws, such as Coulombic ones. Moreover, some physical factors describing granular matter are lacking to the model. The equations that we retrieve are analogous to those of inelastic contact mechanics, while a non-zero restitution coefficient might be necessary to properly model some classes of granular flows.

Finally, as usual when dealing with Coulomb-like friction models, the theoretical existence and uniqueness criterions for our solutions are rather weak. Not being able to uniquely determine the stress field for a given velocity solution would be problematic for assessing the stress on structures. However, as the flowing and dilating regions enforce boundary conditions for the stress inside the rigid zones, we did not find this underdetermination to be problematic for our test scenarios.

\subsection{Conclusion and future work}

In this work we have introduced a macroscopic, continuous model for granular-like flows, and we have shown that it was driven by equations structurally similar to those appearing in contact mechanics between discrete elements. We have then exhibited algorithms for solving those equations on a class of spatial discretization and demonstrated that they were well-defined.

These new numerical simulation tools allowed us to capture the typical nonsmooth effects critical to the simulation of yield-stress flows, as well as to accommodate complex rheologies such as the well-known $\mu(I)$ rheology. The main advantage over most previous approaches is the absence of any regularization or clamping. The formulation in the framework of Second Order Cone Programming paves the way for methods with superlinear convergence rates.

In future work we plan to free ourselves from the dense flow hypothesis and extend our approach to dry flows with varying volume fraction of grains. On the computational side, we would also like to devise more efficient solvers, in order to make three-dimensional scenarios tractable.

Finally, we emphasized in this work the numerical resolution of the discrete problem, but more theoretical considerations would also be of interest. First, one may wonder if the condition $\overline{\mathcal{H}}(\mu)$ for the existence of a discrete solution holds in the continuous limit. We hold good faith that the proof given by Cadoux in [Cadoux, 2009] could be adapted to this case, and will investigate this point more thoroughly in the future. Moreover, Barker et al. [Barker et al., 2015] motivate the modeling of transitions between different regimes of the granular material by suggesting that such transitions may remove an instability revealed in the flowing regime of the $\mu(I)$ rheology. Determining whether our inclusion of solid and gaseous regimes is sufficient for the same purpose represents an interesting research direction.

\section{Acknowledgments}

We would like to thank Pierre-Yves Lagrée for insightful discussions about this work, Pierre Saramito for his help on the use of the Rheolef software, and all the anonymous reviewers for their fruitful comments. This work has been supported in part by the LabEx PERSYVALLab (ANR-11-LABX-0025-01) funded by the French program Investissement d'avenir.

\section{A Proofs}

\section{A.1 Proofs of Properties 1 and 2 on the isomorphism $\chi$}

Property 1. $\chi$ is an orthonormal isomorphism between the two Euclidean spaces $\left(\mathbb{R}^{\mathfrak{s}(d)} ;^{\top} \cdot\right)$ and $\left(S_{d} ;<\cdot, \cdot>\right)$. This means

$$
\forall(\boldsymbol{x}, \boldsymbol{y}) \in \mathbb{R}^{\mathfrak{s}(d)} \times \mathbb{R}^{\mathfrak{s}(d)} \boldsymbol{x}^{\top} \boldsymbol{y}=<\chi(\boldsymbol{x}), \chi(\boldsymbol{y})>,
$$

where $\boldsymbol{x}^{\top} \boldsymbol{y}$ is the usual scalar product on $\mathbb{R}^{m}, m \geq 1$ and $<\boldsymbol{\sigma}, \boldsymbol{\tau}\rangle=\frac{\boldsymbol{\sigma}: \tau}{2}$ is our scalar product on $S_{d}$.

Proof. Let $\|\boldsymbol{x}\|=\sqrt{\boldsymbol{x}^{\top} \boldsymbol{x}}$ be the Euclidean norm of $\boldsymbol{x} \in$ $\mathbb{R}^{m}, m \geq 1$. Direct calculations show that

$$
\begin{array}{rlrl}
|\chi(a ; b, c)|^{2} & =a^{2}+b^{2}+c^{2}=\|(a ; b, c)\|^{2} & & \text { when } d=2 \\
|\chi(a ; b, c, d, e, f)|^{2} & =a^{2}+b^{2}+c^{2}+d^{2}+e^{2}+f^{2} & \\
& =\|(a ; b, c, d, e, f)\|^{2} & \text { when } d=3,
\end{array}
$$

meaning that

$$
\forall \boldsymbol{x} \in \mathbb{R}^{\mathfrak{s}(d)}\|\boldsymbol{x}\|=|\chi(\boldsymbol{x})|
$$


By decomposing the scalar product, we get $\forall(\boldsymbol{x}, \boldsymbol{y}) \in \mathbb{R}^{\mathfrak{s}(d)} \times \mathbb{R}^{\mathfrak{s}(d)}$,

$$
\begin{aligned}
\boldsymbol{x}^{\top} \boldsymbol{y} & =\frac{1}{2}\left(\|\boldsymbol{x}+\boldsymbol{y}\|^{2}-\|\boldsymbol{x}\|^{2}-\|\boldsymbol{y}\|^{2}\right) \\
& =\frac{1}{2}\left(|\chi(\boldsymbol{x}+\boldsymbol{y})|^{2}-|\chi(\boldsymbol{x})|^{2}-|\chi(\boldsymbol{y})|^{2}\right) \text { from }(45) \\
& =<\chi(\boldsymbol{x}), \chi(\boldsymbol{y})>\quad \text { using the linearity of } \chi .
\end{aligned}
$$

Property 2. Let us use the notation $\left(\sigma_{N} ; \boldsymbol{\sigma}_{T}\right):=\overline{\boldsymbol{\sigma}}:=$ $\chi^{-1}(\boldsymbol{\sigma})$ to decompose a symmetric tensor $\boldsymbol{\sigma} \in S_{d}$ or its preimage by $\chi, \overline{\boldsymbol{\sigma}} \in \mathbb{R}^{\mathfrak{s}(d)}$, into a normal component $\sigma_{N} \epsilon$ $\mathbb{R}$ and a tangential component $\boldsymbol{\sigma}_{T} \in \mathbb{R}^{\mathfrak{t}(d)}$. We have

$$
\begin{array}{ll} 
& \frac{1}{\sqrt{2 d}} \operatorname{Tr}(\boldsymbol{\sigma})=\sigma_{N} \\
\text { and } \quad & |\operatorname{Dev}(\boldsymbol{\sigma})|=\left\|\boldsymbol{\sigma}_{T}\right\| .
\end{array}
$$

Proof. By linearity of $\chi$, we have $\boldsymbol{\sigma}=\chi\left(\sigma_{\mathrm{N}} ; \boldsymbol{\sigma}_{\mathrm{T}}\right)=$ $\chi\left(\sigma_{\mathrm{N}} ; 0\right)+\chi\left(0 ; \boldsymbol{\sigma}_{\mathrm{T}}\right)$. Then, by linearity of the trace and by noting from the definition of $\chi$ that $\operatorname{Tr}\left(\chi\left(0 ; \boldsymbol{\sigma}_{\mathrm{T}}\right)\right)=0$, it follows that $\operatorname{Tr}(\boldsymbol{\sigma})=\operatorname{Tr}\left(\chi\left(\sigma_{\mathrm{N}} ; 0\right)\right)=\sqrt{2 d} \sigma_{\mathrm{N}}$.

Likewise, by linearity of the Dev operator and by noting from the definition of $\chi$ that $\operatorname{Dev}\left(\chi\left(\sigma_{\mathrm{N}} ; 0\right)\right)=0$, we have

$$
\begin{aligned}
\operatorname{Dev}(\boldsymbol{\sigma}) & =\operatorname{Dev}\left(\chi\left(0 ; \boldsymbol{\sigma}_{\mathrm{T}}\right)\right) \\
& =\chi\left(0 ; \boldsymbol{\sigma}_{\mathrm{T}}\right)-\frac{1}{d} \mathbf{I} \underbrace{\operatorname{Tr}\left(\chi\left(0 ; \boldsymbol{\sigma}_{\mathrm{T}}\right)\right)}_{=0}
\end{aligned}
$$

and therefore from (45) we get $|\operatorname{Dev}(\boldsymbol{\sigma})|=\left\|\left(0 ; \boldsymbol{\sigma}_{\mathrm{T}}\right)\right\|=$ $\left\|\boldsymbol{\sigma}_{\mathrm{T}}\right\|$.

\section{A.2 Proofs of Theorems $1-3$ on the re- formulations of $\mathcal{D P}\left(\mu, \sigma_{0}\right)$}

Theorem 1. Using the notations of Property 2, the rheology $\mathcal{D P}\left(\mu, \sigma_{0}\right)$ can be equivalently expressed as

$$
\left\{\begin{array}{lr}
\boldsymbol{\lambda}_{T}=-\kappa_{\mu, \boldsymbol{\sigma}_{0}}\left(\lambda_{N}\right) \frac{\mathrm{D}(\mathbf{u})_{T}}{\left\|\mathrm{D}(\mathbf{u})_{T}\right\|} & \text { if } \mathrm{D}(\mathbf{u})_{T} \neq \mathbf{0}(13 \mathrm{a}) \\
\left\|\boldsymbol{\lambda}_{T}\right\| \leq \kappa_{\mu, \boldsymbol{\sigma}_{0}}\left(\lambda_{N}\right) & \text { if } \mathrm{D}(\mathbf{u})_{T}=\mathbf{0},(13 \mathrm{~b}) \\
0 \leq \lambda_{N} \perp \mathrm{D}(\mathbf{u})_{N} \geq 0, & (13 \mathrm{c})
\end{array}\right.
$$

that is,

$$
\begin{aligned}
&(\boldsymbol{\lambda} ; \mathrm{D}(\mathbf{u})) \in \mathcal{D P}\left(\mu, \sigma_{0}\right) \Longleftrightarrow \\
&(\overline{\boldsymbol{\lambda}} ; \overline{\mathrm{D}(\mathbf{u})}) \text { satisfies Problem (13). }
\end{aligned}
$$

Proof. Using (12a) we get $\operatorname{Tr} \boldsymbol{\lambda}=\sqrt{2 d} \lambda_{\mathrm{N}}$. Since $|\operatorname{Dev}(\mathrm{D}(\mathbf{u}))|=\left\|\mathrm{D}(\mathbf{u})_{\mathrm{T}}\right\|$ from $(12 \mathrm{~b})$, we have $\operatorname{Dev}(\mathrm{D}(\mathbf{u}))=\mathbf{0} \Longleftrightarrow \mathrm{D}(\mathbf{u})_{\mathrm{T}}=\mathbf{0}$. Thus, it is straightforward to see that the scalar equation (3b) is equivalent to $(13 \mathrm{~b})$.

Moreover,

$$
\begin{aligned}
& (3 \mathrm{a}) \Longleftrightarrow \\
& \left(\mathrm{D}(\mathbf{u})_{\mathrm{T}} \neq \mathbf{0} \Longrightarrow\left\{\begin{array}{c}
|\operatorname{Dev}(\boldsymbol{\lambda})|=\kappa_{\mu, \boldsymbol{\sigma}_{0}}\left(\lambda_{\mathrm{N}}\right) \\
-\operatorname{Dev}(\boldsymbol{\lambda}) \text { collinear to } \operatorname{Dev}(\mathrm{D}(\mathbf{u})
\end{array}\right.\right.
\end{aligned}
$$

Since $\chi$ is orthonormal, it preserves both norm and collinearity. Therefore,

$$
\begin{aligned}
(3 \mathrm{a}) & \Longleftrightarrow\left(\mathrm{D}(\mathbf{u})_{\mathrm{T}} \neq \mathbf{0} \Longrightarrow\left\{\begin{array}{c}
\left\|\boldsymbol{\lambda}_{\mathrm{T}}\right\|=\kappa_{\mu, \boldsymbol{\sigma}_{0}}\left(\lambda_{\mathrm{N}}\right) \\
-\boldsymbol{\lambda}_{\mathrm{T}} \text { collinear to } \mathrm{D}(\mathbf{u})_{\mathrm{T}}
\end{array}\right)\right. \\
& \Longleftrightarrow(13 \mathrm{a}) .
\end{aligned}
$$

Finally, from Property (12a) the scalar complementarity $(3 \mathrm{c})$ rereads

$$
0 \leq \sqrt{2 d} \mathrm{D}(\mathbf{u})_{\mathrm{N}} \perp \sqrt{\frac{2}{d}} \lambda_{\mathrm{N}} \geq 0,
$$

which is equivalent to (13c) since complementarity is insensitive to strictly positive scaling factors.

Theorem 2. The following equivalence holds,

$$
(\boldsymbol{\lambda} ; \mathrm{D}(\mathbf{u})) \in \mathcal{D} \mathcal{P}\left(\mu, \sigma_{0}\right) \Longleftrightarrow f_{A C}^{\star}(\overline{\boldsymbol{\lambda}}, \overline{\mathrm{D}(\mathbf{u})})=0
$$

Proof. Given Theorem(1), it is enough to show that $f_{\mathrm{AC}}^{\star}(\overline{\boldsymbol{\lambda}}, \overline{\mathrm{D}(\mathbf{u})})=0$ if and only if $(\overline{\boldsymbol{\lambda}} ; \overline{\mathrm{D}(\mathbf{u})})$ satisfies Problem (13). Let us first introduce two lemmas for reinterpreting (13).

Lemma 1. The complementarity condition (13c) can be written in a disjunctive fashion as

$$
(13 c) \Longleftrightarrow\left\{\begin{aligned}
& \lambda_{N} \geq 0 \\
& \mathrm{D}(\mathbf{u})_{N} \geq 0 \\
& \mathrm{D}(\mathbf{u})_{N}=0 \text { if } \lambda_{N}=0
\end{aligned}\right.
$$

Lemma 2. If $\kappa_{\mu, \sigma_{0}}\left(\lambda_{N}\right)>0$, Conditions $(13 a-13 b)$ reread

$$
\begin{aligned}
& (13 a-13 b) \Longleftrightarrow \\
& \left\{\begin{array}{rlrl}
\left\|\boldsymbol{\lambda}_{T}\right\| & \leq \kappa_{\mu, \boldsymbol{\sigma}_{0}}\left(\lambda_{N}\right) & \\
\mathrm{D}(\mathbf{u})_{T} & =-\alpha \boldsymbol{\lambda}_{T}, \alpha \in \mathbb{R}_{+} & & \text {if }\left\|\boldsymbol{\lambda}_{T}\right\|=\kappa_{\mu, \boldsymbol{\sigma}_{0}}\left(\lambda_{N}\right) \\
\mathrm{D}(\mathbf{u})_{T} & =0 & & \text { if }\left\|\boldsymbol{\lambda}_{T}\right\|<\kappa_{\mu, \boldsymbol{\sigma}_{0}}\left(\lambda_{N}\right) .
\end{array}\right.
\end{aligned}
$$

Let us now have a look at the normal part of $f_{\mathrm{AC}}^{\star}$, where $\mathcal{C}=\mathbb{R}_{+}$. Using the normal cone defined in (8), we have $\mathcal{N}_{\mathcal{C}}(0)=\mathbb{R}^{-}$and $\mathcal{N}_{\mathcal{C}}(x>0)=\{0\}$. Therefore, according to $(9)$ we get

$$
\begin{aligned}
x=\Pi_{\mathcal{C}}(x-\xi y) & \Longleftrightarrow y \in-\mathcal{N}_{\mathcal{C}}(x) \\
& \Longleftrightarrow \begin{cases}x \in \mathcal{C} \\
y \in \mathbb{R}_{+} & \text {if } x=0 \\
y=0 & \text { if } x>0 .\end{cases}
\end{aligned}
$$

For $x=\lambda_{\mathrm{N}}, y=\mathrm{D}(\mathbf{u})_{\mathrm{N}}$, and $\xi=\xi_{\mathrm{N}}$, we thus identify using Lemma 1 that

$$
f_{\mathrm{AC}}^{\star}(\overline{\boldsymbol{\lambda}}, \overline{\mathrm{D}(\mathbf{u})})_{\mathrm{N}}=0 \Longleftrightarrow(13 \mathrm{c}),
$$

where $f_{\mathrm{AC}}^{\star}(\overline{\boldsymbol{\lambda}}, \overline{\mathrm{D}(\mathbf{u})})_{\mathrm{N}}$ denotes the first component (normal part) of $f_{\mathrm{AC}}^{\star}(\overline{\boldsymbol{\lambda}}, \overline{\mathrm{D}(\mathbf{u})})$.

Let us now consider the tangential part of $f_{\mathrm{AC}}^{\star}$, where $\mathcal{C}=\mathcal{B}^{\mathrm{t}(d)}\left(\kappa_{\mu, \sigma_{0}}\left(\lambda_{\mathrm{N}}\right)\right)$. Let us first assume that $\kappa)_{i, \sigma_{0}}\left(\lambda_{\mathrm{N}}\right)>0$. We then have $\mathcal{N}_{\mathcal{C}}(\boldsymbol{x} \in \operatorname{Int} \mathcal{C})=\{\boldsymbol{0}\}$ and 
$\mathcal{N}_{\mathcal{C}}(\boldsymbol{x} \in \operatorname{Bd} \mathcal{C})=\left\{\alpha \frac{\boldsymbol{x}}{\|\boldsymbol{x}\|}, \alpha \in \mathbb{R}_{+}\right\}$, where Int $\mathcal{C}$ is the interior of $\mathcal{C}$ and $\operatorname{Bd} \mathcal{C}$ its boundary. Using (9), we get

$$
\begin{aligned}
\boldsymbol{x}=\Pi_{\mathcal{C}}(\boldsymbol{x}-\xi \boldsymbol{y}) & \Longleftrightarrow \boldsymbol{y} \in-\mathcal{N}_{\mathcal{C}}(\boldsymbol{x}) \\
& \Longleftrightarrow \begin{cases}\boldsymbol{x} \in \mathcal{C} & \text { if } \boldsymbol{x} \in \operatorname{Int} \mathcal{C} \\
\boldsymbol{y}=\mathbf{0} & \text { if } \boldsymbol{x} \in \operatorname{Bd} \mathcal{C} \\
\boldsymbol{y}=-\alpha \frac{\boldsymbol{x}}{\|\boldsymbol{x}\|}, \alpha \in \mathbb{R}_{+} & \end{cases}
\end{aligned}
$$

For $\boldsymbol{x}=\boldsymbol{\lambda}_{\mathrm{T}}$ and $\boldsymbol{y}=\mathrm{D}(\mathbf{u})_{\mathrm{T}}$, we thus identify using Lemma 2 that

$$
f_{\mathrm{AC}}^{\star}(\overline{\boldsymbol{\lambda}}, \overline{\mathrm{D}(\mathbf{u})})_{\mathrm{T}}=0 \Longleftrightarrow(13 \mathrm{a}-13 \mathrm{~b}) .
$$

where $f_{\mathrm{AC}}^{\star}(\overline{\boldsymbol{\lambda}}, \overline{\mathrm{D}(\mathbf{u})})_{\mathrm{T}}$ denotes the $\mathfrak{t}(d)$ last components (tangential part) of $f_{\mathrm{AC}}^{\star}(\overline{\boldsymbol{\lambda}}, \overline{\mathrm{D}(\mathbf{u})})$. In the degenerate case where $\kappa_{\mu, \boldsymbol{\sigma}_{0}}\left(\lambda_{\mathrm{N}}\right)=0$, we have $C=\{\mathbf{0}\}$ and $\mathcal{N}_{\mathcal{C}}(\boldsymbol{x} \in \mathcal{C})=$ $\mathbb{R}^{\mathfrak{t}(d)}$. We thus trivially obtain $\boldsymbol{x}=\Pi_{\mathcal{C}}(\boldsymbol{x}-\xi \boldsymbol{y}) \Longleftrightarrow \boldsymbol{x}=$ $\mathbf{0}$, the latter equality being what Equations $(13 \mathrm{a}-13 \mathrm{~b})$ boil down to for $\boldsymbol{x}=\boldsymbol{\lambda}_{\mathrm{T}}$ and $\boldsymbol{y}=\mathrm{D}(\mathbf{u})_{\mathrm{T}}$ when $\kappa_{\mu, \boldsymbol{\sigma}_{0}}\left(\lambda_{\mathrm{N}}\right)=$ 0 . We thus again retrieve the above equivalence (46).

Theorem 3. The following equivalence holds,

$$
(\boldsymbol{\lambda} ; \mathrm{D}(\mathbf{u})) \in \mathcal{D} \mathcal{P}\left(\mu, \sigma_{0}\right) \Longleftrightarrow f_{D S}^{\star}(\overline{\boldsymbol{\lambda}}, \overline{\mathrm{D}(\mathbf{u})})=\mathbf{0} .
$$

Proof. For the sake of brevity, we only prove Theorem 19 in the case $\sigma_{0}>0$. The case $\sigma_{0}=0$ and Corollary (1) reduce to the standard Signorini-Coulomb law and are dealt with, for instance, in [Cadoux, 2009].

Again, given Theorem 1 , it is enough to show that $f_{\mathrm{DS}}^{\star}(\overline{\boldsymbol{\lambda}}, \overline{\mathrm{D}(\mathbf{u})})=\mathbf{0}$ if and only if $(\overline{\boldsymbol{\lambda}} ; \overline{\mathrm{D}(\mathbf{u})})$ satisfies Problem (13). Let us first notice that the following equivalences hold,

$$
\begin{aligned}
& \overline{\hat{\mathrm{D}}(\mathbf{u})} \in \mathcal{K}_{\frac{1}{\mu}}^{\mathfrak{s}(d)} \Longleftrightarrow \mathrm{D}(\mathbf{u})_{\mathrm{N}} \geq 0 \\
& \overline{\hat{\mathrm{D}}(\mathbf{u})} \in \mathrm{Bd} \mathcal{K}_{\frac{1}{\mu}}^{\mathfrak{s}(d)} \Longleftrightarrow \mathrm{D}(\mathbf{u})_{\mathrm{N}}=0
\end{aligned}
$$$$
\text { and } \overline{\hat{\mathrm{D}}(\mathbf{u})}=\mathbf{0} \Longleftrightarrow \overline{\mathrm{D}(\mathbf{u})}=\mathbf{0} \text {. }
$$

Moreover, since $\sigma_{0}>0$ implies that $\kappa_{\mu, \sigma_{0}}\left(\lambda_{\mathrm{N}}\right)>0$, we can use Lemmas 1 and 2 to write Problem (13) equivalently as

$$
\begin{aligned}
& (13) \Longleftrightarrow \\
& \left\{\begin{array}{rr}
\overline{\boldsymbol{\lambda}} \in \mathcal{K}_{\mu, \sigma_{0}} & \\
\mathrm{D}(\mathbf{u})_{\mathrm{T}}=-\alpha \boldsymbol{\lambda}_{\mathrm{T}}, \alpha \in \mathbb{R}_{+} & \text {if }\left\|\boldsymbol{\lambda}_{\mathrm{T}}\right\|=\kappa_{\mu, \boldsymbol{\sigma}_{0}}\left(\lambda_{\mathrm{N}}\right) \\
\mathrm{D}(\mathbf{u})_{\mathrm{T}}=0 & \text { if }\left\|\boldsymbol{\lambda}_{\mathrm{T}}\right\|<\kappa_{\mu, \boldsymbol{\sigma}_{0}}\left(\lambda_{\mathrm{N}}\right) \\
\mathrm{D}(\mathbf{u})_{\mathrm{N}} \geq 0 & \text { if } \lambda_{\mathrm{N}}=0 \\
\mathrm{D}(\mathbf{u})_{\mathrm{N}}=0 & \text { if } \lambda_{\mathrm{N}}>0 .
\end{array}\right.
\end{aligned}
$$

Now, from the projection theorem (9), we have

$$
f_{\mathrm{DS}}^{\star}(\overline{\boldsymbol{\lambda}}, \overline{\mathrm{D}(\mathbf{u})})=\mathbf{0} \Longleftrightarrow \overline{\hat{\mathrm{D}}(\mathbf{u})} \in-\mathcal{N}_{\mathcal{K}_{\mu, \sigma_{0}}^{\mathfrak{s}(d)}}(\overline{\boldsymbol{\lambda}}) .
$$

Let us specify the form of the normal cone $\mathcal{N}_{\mathcal{C}}(\bar{\lambda})$ with $\mathcal{C}:=\mathcal{K}_{\mu, \sigma_{0}}^{\mathfrak{s}(d)}$, for the four different cases of $\overline{\boldsymbol{\lambda}} \in \mathcal{C}$. i. $\lambda_{\mathbf{N}}>0$ and $\left\|\lambda_{\mathbf{T}}\right\|<\kappa_{\mu, \sigma_{0}}\left(\lambda_{\mathbf{N}}\right)$, i.e., $\bar{\lambda} \in \operatorname{Int} \mathcal{C}$ Then $\mathcal{N}_{\mathcal{C}}(\overline{\boldsymbol{\lambda}})=\{\mathbf{0}\}$ and from $(47 \mathrm{c})$ we get

$$
\overline{\hat{\mathrm{D}}(\mathbf{u})} \in-\mathcal{N}_{\mathcal{C}}(\overline{\boldsymbol{\lambda}}) \Longleftrightarrow \overline{\boldsymbol{\lambda}} \in \mathcal{C}, \overline{\mathrm{D}(\mathbf{u})}=\mathbf{0} .
$$

ii. $\lambda_{\mathbf{N}}>0$ and $\left\|\boldsymbol{\lambda}_{\mathbf{T}}\right\|=\kappa_{\mu, \sigma_{0}}\left(\lambda_{\mathrm{N}}\right)$, i.e., $\overline{\boldsymbol{\lambda}}$ lies on the smooth conical part of $\mathrm{Bd} \mathcal{C}$ The outward normal to $\mathcal{C}$ at $\overline{\boldsymbol{\lambda}}$ reads $\mathbf{n}_{\mathcal{C}}(\overline{\boldsymbol{\lambda}})=\frac{1}{\sqrt{1+\mu^{2}}}\left(-\mu ; \frac{\boldsymbol{\lambda}_{\mathrm{T}}}{\left\|\boldsymbol{\lambda}_{\mathrm{T}}\right\|}\right)$, and thus we have $\mathcal{N}_{\mathcal{C}}(\overline{\boldsymbol{\lambda}})=\left\{\alpha\left(-\mu ; \frac{\boldsymbol{\lambda}_{\mathrm{T}}}{\left\|\boldsymbol{\lambda}_{\mathrm{T}}\right\|}\right), \alpha \in \mathbb{R}_{+}\right\} \subset-\operatorname{Bd} \mathcal{K}_{\frac{1}{\mu}}^{\mathfrak{s}(d)}$. Using $(47 \mathrm{~b})$ and $(17 \mathrm{~b})$, we get

$$
\overline{\hat{\mathrm{D}}(\mathbf{u})} \in-\mathcal{N}_{\mathcal{C}}(\overline{\boldsymbol{\lambda}}) \Longleftrightarrow\left\{\begin{array}{l}
\overline{\boldsymbol{\lambda}} \in \mathcal{C} \\
\mathrm{D}(\mathbf{u})_{\mathrm{N}}=0 \\
\mathrm{D}(\mathbf{u})_{\mathrm{T}}=-\alpha \boldsymbol{\lambda}_{\mathrm{T}}, \alpha \in \mathbb{R}_{+} .
\end{array}\right.
$$

iii. $\quad \lambda_{\mathbf{N}}=0$ and $\left\|\lambda_{\mathbf{T}}\right\|<\kappa_{\mu, \sigma_{0}}\left(\lambda_{\mathbf{N}}\right)$, i.e., $\bar{\lambda}$ lies on the smooth planar base of $\mathbf{B d} \mathcal{C}$ We have $\mathbf{n}_{\mathcal{C}}(\overline{\boldsymbol{\lambda}})=$ $(-1 ; \mathbf{0})$ and then $\mathcal{N}_{\mathcal{C}}(\overline{\boldsymbol{\lambda}})=\left\{\alpha(-1 ; \mathbf{0}), \alpha \in \mathbb{R}_{+}\right\}$. Thus,

$\overline{\hat{\mathrm{D}}(\mathbf{u})} \in-\mathcal{N}_{\mathcal{C}}(\overline{\boldsymbol{\lambda}}) \Longleftrightarrow \bar{\lambda} \in \mathcal{C}, \mathrm{D}(\mathbf{u})_{\mathrm{T}}=0$ and $\mathrm{D}(\mathbf{u})_{\mathrm{N}} \geq 0$.

iv. $\lambda_{\mathbf{N}}=0$ and $\left\|\lambda_{\mathbf{T}}\right\|=\kappa_{\mu, \sigma_{0}}\left(\lambda_{\mathbf{N}}\right)$, i.e., $\bar{\lambda}$ lies on the nonsmooth circular part of $\operatorname{Bd} \mathcal{C}$ Then $\mathcal{N}_{\mathcal{C}}(\bar{\lambda})=$ $-\mathcal{K}_{\frac{1}{\mu}}^{\mathfrak{s}(d)} \cap\left\{\boldsymbol{\gamma} \in \mathbb{R}^{\mathfrak{s}(d)}, \boldsymbol{\gamma}_{\mathrm{T}}=\alpha \boldsymbol{\lambda}_{\mathrm{T}}, \alpha \in \mathbb{R}_{+}\right\}$. Again, from (47a) and ${ }^{\mu}(17 b)$,

$$
\begin{aligned}
& \overline{\hat{\mathrm{D}}(\mathbf{u})} \in-\mathcal{N}_{\mathcal{C}}(\overline{\boldsymbol{\lambda}}) \Longleftrightarrow \\
& \overline{\boldsymbol{\lambda}} \in \mathcal{C}, \mathrm{D}(\mathbf{u})_{\mathrm{N}} \geq 0 \text { and } \mathrm{D}(\mathbf{u})_{\mathrm{T}}=-\alpha \boldsymbol{\lambda}_{\mathrm{T}}, \alpha \in \mathbb{R}_{+} .
\end{aligned}
$$

Finally, it is straightforward to see that the union of the four cases $i, i i, i i i, i v$ exactly amounts to our conditions derived in (48).

\section{A.3 Discrete problem (34) on quadrature points}

Property 3. Let $\breve{\boldsymbol{\Lambda}}_{h}:=R \boldsymbol{\Lambda}_{h}$ and $\breve{\boldsymbol{\Gamma}}_{h}:=R \boldsymbol{\Gamma}_{h}$, and $R^{\dagger}$ the Moore-Penrose pseudoinverse of $R$. Then Equations (25a - 25c) can be discretized as:

Find $\mathbf{U}_{h} \in \mathbb{R}^{\mathfrak{v}}, \breve{\Lambda}_{h}, \breve{\Gamma}_{h} \in \mathbb{R}^{\mathfrak{s}(d) n_{Q}}$,

$$
\left\{\begin{array}{c}
A \mathbf{U}_{h}=B^{\top} R^{\dagger} \breve{\Lambda}_{h}+\mathbf{l}_{t o t} \\
\breve{\boldsymbol{\Gamma}}_{h}=R^{\dagger, \top} B \mathbf{U}_{h}+R^{\dagger, \top} \mathbf{k}_{t o t} \\
\forall 1 \leq q \leq n_{Q}, \quad f_{D S}^{\star}\left(\breve{\Lambda}_{h}[q], \breve{\boldsymbol{\Gamma}}_{h}[q]\right)=\mathbf{0} .
\end{array}\right.
$$

Proof. First, it is noteworthy that matrix $M$ may be decomposed as $M=R^{T} \operatorname{diag}(S) R$, where $\operatorname{diag}(S)$ is a diagonal matrix of size $\mathfrak{s}(d) n_{Q}$ mapping the diagonal weight block $\mathfrak{S}_{q, q}:=2 w_{q} I_{\mathfrak{s}(d)}$ to each quadrature point $q$, that is, whose diagonal coefficients $S$ are given by

$S_{(q-1) \mathfrak{s}(d)+k}=2 w_{q} \quad$ for $1 \leq k \leq \mathfrak{s}(d) \quad$ and $\quad 1 \leq q \leq n_{Q}$. 
Indeed, for all $1 \leq i, j \leq n$ and for all $1 \leq k, \ell \leq n$ let $r:=(i-1) \mathfrak{s}(d)+k$ and $c:=(j-1) \mathfrak{s}(d)+\ell$. Using the quadrature rule, we have

$$
\begin{aligned}
M_{r, c} & =\sum_{q=1}^{n_{Q}} w_{q}\left(\boldsymbol{\sigma}_{r}\left(\breve{x}_{q}\right): \boldsymbol{\sigma}_{c}\left(\breve{x}_{q}\right)\right) \\
& =2 \sum_{q=1}^{n_{Q}} w_{q}\left(\overline{\boldsymbol{\sigma}}_{r}\left(\breve{x}_{q}\right)^{\top} \overline{\boldsymbol{\sigma}}_{c}\left(\breve{x}_{q}\right)\right) \quad \text { from (11) } \\
& =2 \sum_{q=1}^{n_{Q}} w_{q}\left(\alpha_{i}\left(\breve{x}_{q}\right) \mathbf{e}_{k}^{\top} \alpha_{j}\left(\breve{x}_{q}\right) \mathbf{e}_{\ell}\right) \\
& =2 \sum_{q=1}^{n_{Q}} w_{q}\left(\alpha_{i}\left(\breve{x}_{q}\right) \alpha_{j}\left(\breve{x}_{q}\right) \delta_{k, \ell}\right) \\
& =2 \sum_{q=1}^{n_{Q}} \sum_{p=1}^{\mathfrak{s}(d)} w_{q}\left(\alpha_{i}\left(\breve{x}_{q}\right) \alpha_{j}\left(\breve{x}_{q}\right) \delta_{k, p} \delta_{p, l}\right) \\
& =\sum_{q=1}^{n_{Q}} \sum_{p=1}^{\mathfrak{s}(d)} S_{(q-1) \mathfrak{s}(d)+p}\left(R_{(q-1) \mathfrak{s}(d)+p, r} R_{(q-1) \mathfrak{s}(d)+p, c}\right)
\end{aligned}
$$

which we recognize as the product $M=R^{\top} \operatorname{diag}(S) R$.

Furthermore, since $M$ is invertible, we have $\operatorname{rank}(M)=$ $\mathfrak{s}(d) n$. From the rank inequality on product of matrices, we get

$$
\operatorname{rank}(M) \leq \min (\operatorname{rank}(\operatorname{diag}(S)), \operatorname{rank}(R)) \leq \operatorname{rank}(R),
$$

meaning that the rank of $R$ is equal to its number of columns $\mathfrak{s}(d) n$. The pseudoinverse $R^{\dagger}$ can therefore be computed as $R^{\dagger}=\left(R^{\top} R\right)^{-1} R^{\top}$, and $R^{\dagger}$ plays the role of a left inverse, i.e., we have $R^{\dagger} R=\mathbf{I}$.

We thus have $R^{\dagger} \breve{\Lambda}_{h}=R^{\dagger} R \boldsymbol{\Lambda}_{h}=\boldsymbol{\Lambda}_{h}$, so (34a) is directly equivalent to (32a). Moreover, since for any positive scalar $\xi, f_{\mathrm{DS}}^{\star}(\mathbf{x}, \mathbf{y})=\mathbf{0} \Longleftrightarrow f_{\mathrm{DS}}^{\star}(\mathbf{x}, \xi \mathbf{y})=\mathbf{0}$, the system is not affected by a multiplication of the left-handside of (34b) with the matrix $\operatorname{diag}(S)$. Multiplying then both sides of (34b) by $R^{\top}$, we get

$$
R^{\top} \operatorname{diag}(S) \breve{\boldsymbol{\Gamma}}_{h}=R^{\top} R^{\dagger, \top} B \mathbf{U}_{h}+R^{\top} R^{\dagger, \top} \mathbf{k}_{t o t}
$$

Since $R^{\top} R^{\dagger, \top}=\left(R^{\dagger} R\right)^{\top}=\mathbf{I}$, we retrieve $(32 \mathrm{~b})$.

Finally, as $\breve{\Lambda}_{h}[q]=\overline{\boldsymbol{\lambda}_{h}}\left(\breve{x}_{q}\right)$ and $\breve{\boldsymbol{\Gamma}}_{h}[q]=\overline{\gamma_{h}}\left(\breve{x}_{q}\right)$, we have $(34 c) \Longleftrightarrow\left(\boldsymbol{\lambda}_{h}\left(\breve{x}_{q}\right) ; \gamma_{h}\left(\breve{x}_{q}\right)\right) \in \mathcal{D P}(\mu, \mathrm{Bi})$, that is, we ensure that $\forall \boldsymbol{\tau} \in T_{h}$ the integral (33) is zero, and thus that the projection of the rheology constraint on $T_{h}$ is satisfied everywhere.

\section{A.4 Proof of Theorem 4 about the dual- ity with $\mathrm{Bi}>0$}

Theorem 4. We introduce a modification of the objective function of the primal problem (39),

$$
J_{\mathrm{Bi}}^{*}(\mathbf{u}):=J^{*}(\mathbf{u})+\mathrm{Bi} \sum_{i=1}^{n_{Q}}\left\|\left(R^{\dagger, \top}(B \mathbf{u}+\mathbf{k})\right)_{T}^{i}\right\|,
$$

and the two optimization problems,

$$
\begin{aligned}
& \min _{\mathbf{u} \in C(s)} J_{\mathrm{Bi}}^{\star}(\mathbf{u}) \\
& \min _{\boldsymbol{\lambda} \in \mathcal{K}_{\mu, \mathrm{Bi}}} J_{s}(\boldsymbol{\lambda}) .
\end{aligned}
$$

Then, under the hypothesis that $\operatorname{Int} C(s) \neq 0$, it holds that (42) and (43) are dual problems, in the sense of Fenchel. Moreover, the optimal solution $\mathbf{u}$ of (42) and any optimal solution $\boldsymbol{\lambda}$ of (43) satisfy

$$
\left\{\begin{aligned}
A \mathbf{u} & =B^{\top} R^{\dagger} \boldsymbol{\lambda}+\mathbf{l} \\
\hat{\gamma} & =R^{\dagger, \top} B \mathbf{u}+R^{\dagger, \top} \mathbf{k}+(s ; 0) \\
\boldsymbol{\lambda} & =\Pi_{\mathcal{K}_{\mu, \text { Bi }}}(\boldsymbol{\lambda}-\xi \hat{\gamma}) .
\end{aligned}\right.
$$

Proof. Let us introduce the function $g: \mathbb{R}^{n_{Q} \mathfrak{s}(d)} \rightarrow \mathbb{R}, \gamma \mapsto$ $\sum_{i} \mathrm{Bi}\left\|\gamma_{\mathrm{T}}^{i}\right\|$, and the bounded affine map $L: \mathbb{R}^{\mathfrak{v}} \rightarrow \mathbb{R}^{n_{Q} \mathfrak{s}(d)}$, $\mathbf{u} \mapsto R^{\dagger, \mathrm{T}}(B \mathbf{u}+\mathbf{k})+(s ; 0)$. Problem (42) is equivalent to

$$
\min _{\mathbf{u} \in \mathbb{R}^{\mathfrak{v}}} J^{*}(\mathbf{u})+\left(g+\mathcal{I}_{\mathcal{K}_{\frac{1}{\mu}}}\right) \circ L(\mathbf{u})
$$

As $J^{*}$ and $g+\mathcal{I}_{\mathcal{K}_{\frac{1}{\mu}}}$ are both convex, the hypothesis Int $C(s) \neq 0$ allows us to apply Fenchel's duality theorem and obtain strong duality of (42) and (51),

$$
\begin{aligned}
-\min _{\boldsymbol{\lambda} \in \mathbb{R}^{n_{Q^{\mathfrak{s}}(d)}}}\left(J^{*}\right)^{\star}\left(B^{\top} R^{\dagger} \boldsymbol{\lambda}\right) & +\left(g+\mathcal{I}_{\mathcal{K}_{\frac{1}{\mu}}}\right)^{\star}(\boldsymbol{\lambda}) \\
& -\left(R^{\dagger, \top} \mathbf{k}+(s ; 0)\right)^{\top} \boldsymbol{\lambda},
\end{aligned}
$$

where the $f^{\star}$ notation denotes the convex conjugate of a convex function $f$,

$$
f^{\star}(\mathbf{z}):=\sup _{\mathbf{u} \in \mathbb{R}^{\mathfrak{v}}}(\mathbf{u} \cdot \mathbf{z}-f(\mathbf{u}))
$$

Straightforward computations yield

$$
\begin{aligned}
\left(J^{*}\right)^{\star}(\mathbf{z}) & =\frac{1}{2}(\mathbf{z}+\mathbf{l})^{\top} A^{-1}(\mathbf{z}+\mathbf{l}) & \forall \mathbf{z} \in \mathbb{R}^{\mathfrak{v}} \\
\left(g+\mathcal{I}_{\mathcal{K}_{\frac{1}{\mu}}}\right)^{\star}(\boldsymbol{\lambda}) & =\mathcal{I}_{-\mathcal{K}_{\mu, \mathrm{Bi}}}(\boldsymbol{\lambda}) & \forall \boldsymbol{\lambda} \in \mathbb{R}^{n_{Q} \mathfrak{s}(d)} .
\end{aligned}
$$

We can therefore rewrite (51) as

${ }_{-} \min _{-\boldsymbol{\lambda} \in \mathcal{K}_{\mu, \mathrm{Bi}}}\left(\mathbf{l}+B^{\top} R^{\dagger} \boldsymbol{\lambda}\right) A^{-1}\left(\mathbf{l}+B^{\top} R^{\dagger} \boldsymbol{\lambda}\right)-\left(R^{\dagger, \top} \mathbf{k}+(s ; 0)\right)^{\top} \boldsymbol{\lambda}$.

Developing, we obtain

$$
\left(-\min _{\boldsymbol{\lambda} \in \mathcal{K}_{\mu, \mathrm{Bi}}} J_{s}(\boldsymbol{\lambda})\right)-\frac{1}{2} \mathbf{l} A^{-1} \mathbf{l} .
$$

This means that the dual problem (43) admits a solution $\boldsymbol{\lambda}$, from which we can easily reconstruct a velocity solution as $\mathbf{u}=A^{-1}\left(B^{\top} R^{\dagger} \boldsymbol{\lambda}+\mathbf{l}\right)$; we can then readily verify that the couple $(\mathbf{u}, \boldsymbol{\lambda})$ satisfies System (44), and therefore that $\mathbf{u}$ is the unique solution of the strictly convex primal (42).

\section{B Velocity and stress field in a discharging silo}

This appendix contains visualizations of the steady-state velocity and stress fields for a discharging silo, in both the creeping flow setting of Section 4 and the inertial regime of Section 5. The geometry described in Figure 8 was slightly extended below the outlet.

Results are shown in Figures 16, 17 and 18. 


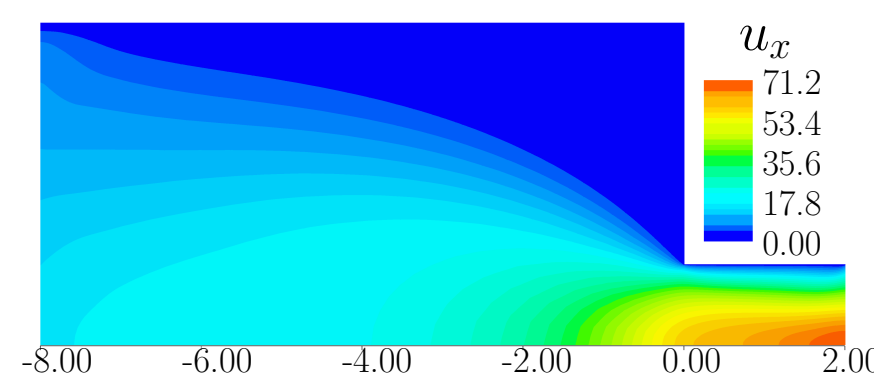

(a) Without inertia

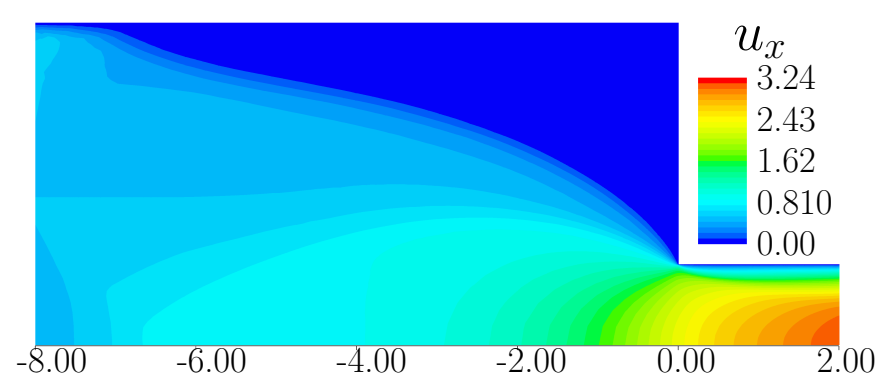

(b) With inertia

Figure 16: Axial velocity $u_{x}$ for simulations with and without inertia for $\operatorname{Re}=100, \mu=0.3$ and $\mathrm{Bi}=0$.

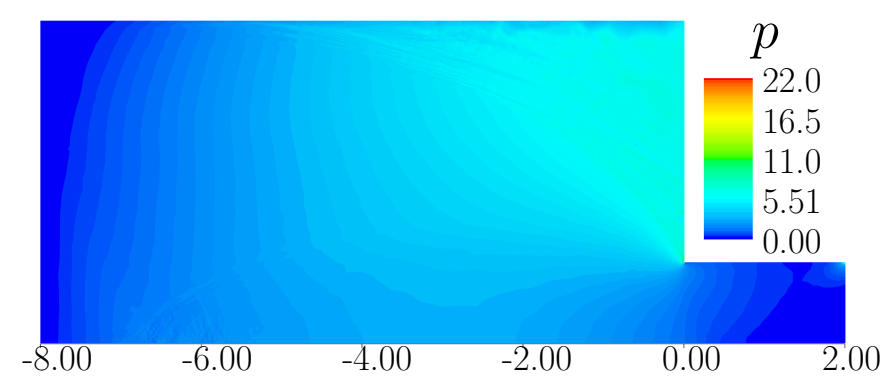

(a) Without inertia

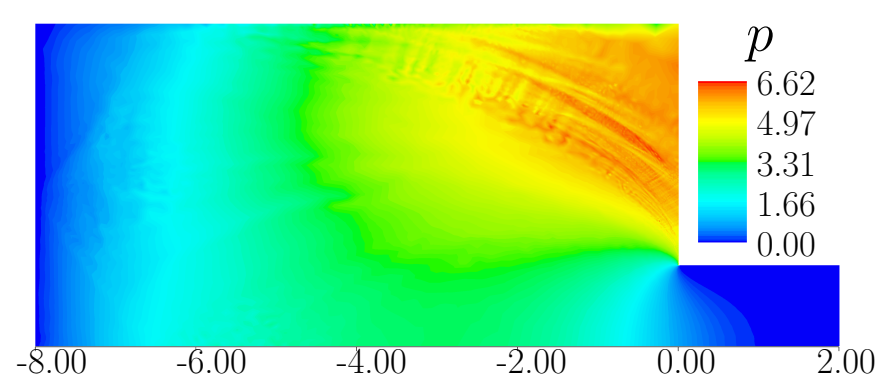

(b) With inertia

Figure 17: Pressure fields for simulations with and without inertia for $\mathrm{Re}=100, \mu=0.3$ and $\mathrm{Bi}=0$. In the creeping flow case, there exist a very high pressure point on the boundary of the outlet. In the inertial case, the high-pressure zone on the bottom of the silo starts at some distance from the outlet and is much more widely spread.

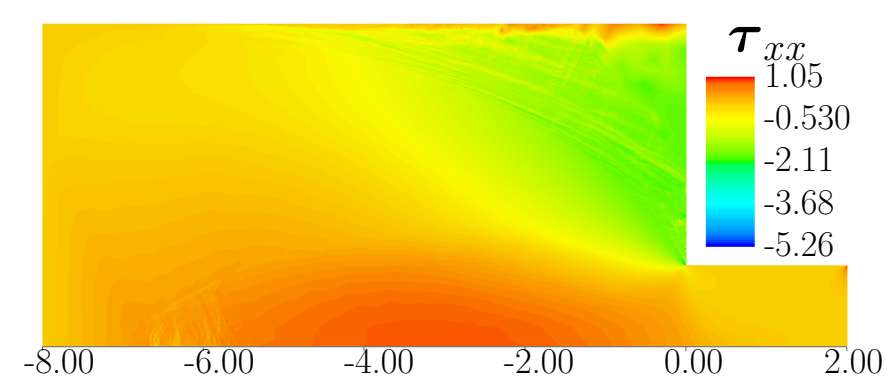

(a) Without inertia

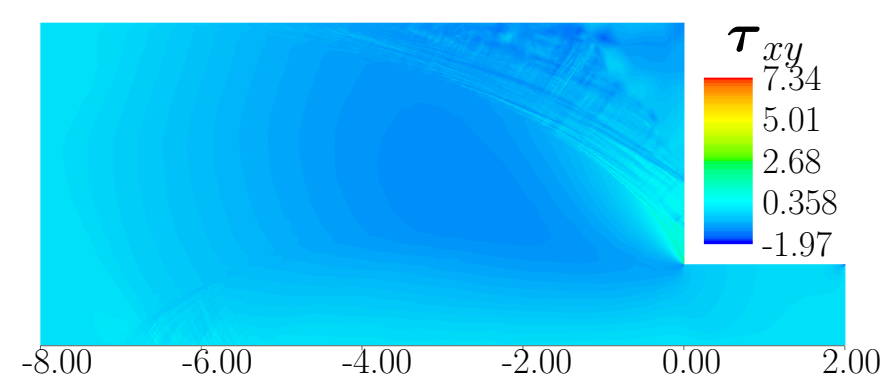

(c) Without inertia

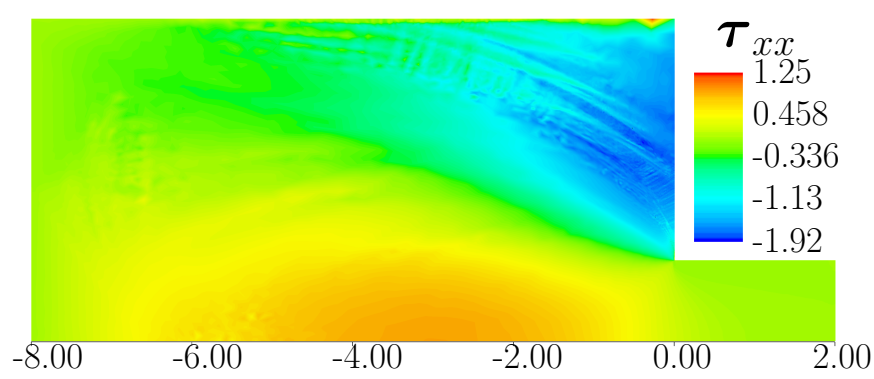

(b) With inertia

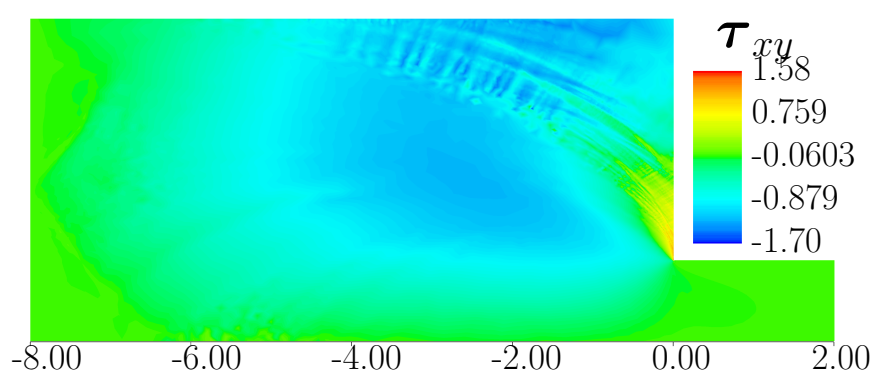

(d) With inertia

Figure 18: Normal (top) and tangential (bottom) components of the deviatoric stress tensor $\tau$ for simulations with and without inertia for $\mathrm{Re}=100, \mu=0.3$ and $\mathrm{Bi}=0$. 


\section{References}

[Acary et al., 2011] Acary, V., Cadoux, F., Lemaréchal, C., and Malick, J. (2011). A formulation of the linear discrete coulomb friction problem via convex optimization. ZAMM - Journal of Applied Mathematics and Mechanics, 91(2):155-175.

[Alart and Curnier, 1991] Alart, P. and Curnier, A. (1991). A mixed formulation for frictional contact problems prone to Newton like solution methods. Computer Methods in Applied Mechanics and Engineering, $92(3): 353-375$.

[Alart et al., 2012] Alart, P., Iceta, D., and Dureisseix, D. (2012). A nonlinear domain decomposition formulation with application to granular dynamics. Computer Methods in Applied Mechanics and Engineering, 205-208:59-67.

[Andersen et al., 2003] Andersen, E., Roos, C., and Terlaky, T. (2003). On implementing a primal-dual interior-point method for conic quadratic optimization. Mathematical Programming, 95(2):249-277.

[Bardenhagen et al., 2000] Bardenhagen, S., Brackbill, J., and Sulsky, D. (2000). The material-point method for granular materials. Computer Methods in Applied Mechanics and Engineering, 187(3-4):529-541.

[Barker et al., 2015] Barker, T., Schaeffer, D., Bohorquez, P., and Gray, J. (2015). Well-posed and ill-posed behaviour of the -rheology for granular flow. Journal of Fluid Mechanics, 779:794-818.

[Barzilai and Borwein, 1988] Barzilai, J. and Borwein, J. M. (1988). Two-point step size gradient methods. IMA Journal of Numerical Analysis, 8(1):141-148.

[Beverloo et al., 1961] Beverloo, W., Leniger, H., and van de Velde, J. (1961). The flow of granular solids through orifices. Chemical Engineering Science, 15(34):260-269.

[Bleyer et al., 2015] Bleyer, J., Maillard, M., de Buhan, P., and Coussot, P. (2015). Efficient numerical computations of yield stress fluid flows using second-order cone programming. Computer Methods in Applied Mechanics and Engineering, 283:599-614.

[Cadoux, 2009] Cadoux, F. (2009). Optimization and convex analysis for nonsmooth dynamics. Theses, Université Joseph-Fourier - Grenoble I.

[Chambon et al., 2011] Chambon, G., Bouvarel, R., Laigle, D., and Naaim, M. (2011). Numerical simulations of granular free-surface flows using smoothed particle hydrodynamics. Journal of Non-Newtonian Fluid Mechanics, 166(12-13):698-712.

[Chauchat and Médale, 2014] Chauchat, J. and Médale, M. (2014). A three-dimensional numerical model for dense granular flows based on the mu(i) rheology. Journal of Computational Physics, 256:696-712.
[Chehata et al., 2003] Chehata, D., Zenit, R., and Wassgren, C. R. (2003). Dense granular flow around an immersed cylinder. Physics of Fluids, 15(6):1622.

[Daviet et al., 2011] Daviet, G., Bertails-Descoubes, F., and Boissieux, L. (2011). A hybrid iterative solver for robustly capturing Coulomb friction in hair dynamics. ACM Transactions on Graphics, 30(6):1.

[De Saxcé and Feng, 1998] De Saxcé, G. and Feng, Z.Q. (1998). The bipotential method: A constructive approach to design the complete contact law with friction and improved numerical algorithms. Mathematical and Computer Modelling, 28(4-8):225-245.

[Domnik and Pudasaini, 2012] Domnik, B. and Pudasaini, S. P. (2012). Full two-dimensional rapid chute flows of simple viscoplastic granular materials with a pressure-dependent dynamic slip-velocity and their numerical simulations. Journal of Non-Newtonian Fluid Mechanics, 173-174:72-86.

[Dunatunga and Kamrin, 2015] Dunatunga, S. and Kamrin, K. (2015). Continuum modelling and simulation of granular flows through their many phases. Journal of Fluid Mechanics, 779:483-513.

[Fortin and Glowinski, 1982] Fortin, M. and Glowinski, R. (1982). Méthodes de lagrangien augmenté. application à la résolution numérique de problèmes aux limites. Collection Méthodes Mathématiques de l'Informatique. Dunod.

[Frigaard and Nouar, 2005] Frigaard, I. and Nouar, C. (2005). On the usage of viscosity regularisation methods for visco-plastic fluid flow computation. Journal of Non-Newtonian Fluid Mechanics, 127(1):1-26.

[GDR MiDi, 2004] GDR MiDi (2004). On dense granular flows. The European Physical Journal E, 14(4):341365 .

[Haslinger and Tvrdỳ, 1983] Haslinger, J. and Tvrdỳ, M. (1983). Approximation and numerical solution of contact problems with friction. Aplikace matematiky, 28(1):55-71.

[Heyn, 2013] Heyn, T. D. (2013). On the modeling, simulation, and visualization of many-body dynamics problems with friction and contact. PhD thesis, THE UNIVERSITY OF WISCONSIN-MADISON.

[Hiriart-Urruty and Lemaréchal, 2001] Hiriart-Urruty, J.-B. and Lemaréchal, C. (2001). Fundamentals of convex analysis. Grundlehren text editions. Springer, Berlin, New York.

[Ionescu et al., 2015] Ionescu, I. R., Mangeney, A., Bouchut, F., and Roche, O. (2015). Viscoplastic modeling of granular column collapse with pressuredependent rheology. Journal of Non-Newtonian Fluid Mechanics. 
[Jean, 1999] Jean, M. (1999). The non-smooth contact dynamics method. Computer Methods in Applied Mechanics and Engineering, 177(3-4):235-257.

[Jop et al., 2006] Jop, P., Forterre, Y., and Pouliquen, O. (2006). A constitutive law for dense granular flows. Nature, 441(7094):727-730.

[Jourdan et al., 1998] Jourdan, F., Alart, P., and Jean, M. (1998). A Gauss-Seidel like algorithm to solve frictional contact problems. Computer Methods in Applied Mechanics and Engineering, 155:31-47.

[Kamrin, 2010] Kamrin, K. (2010). Nonlinear elastoplastic model for dense granular flow. International Journal of Plasticity, 26(2):167-188.

[Kleinert et al., 2014] Kleinert, J., Simeon, B., and Obermayr, M. (2014). An inexact interior point method for the large-scale simulation of granular material. Computer Methods in Applied Mechanics and Engineering, 278:567-598.

[Lagrée et al., 2011] Lagrée, P.-Y., Staron, L., and Popinet, S. (2011). The granular column collapse as a continuum: validity of a two-dimensional navier-stokes model with a $\mu(i)$-rheology. Journal of Fluid Mechanics, 686:378-408.

[Lim et al., 2014] Lim, K.-W., Krabbenhoft, K., and Andrade, J. E. (2014). A contact dynamics approach to the granular element method. Computer Methods in Applied Mechanics and Engineering, 268:557-573.

[Mankoc et al., 2007] Mankoc, C., Janda, A., Arévalo, R., Pastor, J. M., Zuriguel, I., Garcimartín, A., and Maza, D. (2007). The flow rate of granular materials through an orifice. Granular Matter, 9(6):407-414.

[Moreau, 1999] Moreau, J. (1999). Numerical aspects of the sweeping process. Computer Methods in Applied Mechanics and Engineering, 177(3-4):329 - 349.

[Nesterov, 1983] Nesterov, Y. (1983). A method of solving a convex programming problem with convergence rate o (1/k2). Soviet Mathematics Doklady, 27(2):372376.

[Renouf and Alart, 2005] Renouf, M. and Alart, P. (2005). Conjugate gradient type algorithms for frictional multi-contact problems: applications to granular materials. Computer Methods in Applied Mechanics and Engineering, 194(18-20):2019-2041.

[Saramito, 2012] Saramito, P. (2012). Efficient C++ finite element computing with Rheolef.

[Saramito, 2015] Saramito, P. (2015). Efficient C++ finite element computing with Rheolef. Lecture.

[Saramito and Roquet, 2001] Saramito, P. and Roquet, N. (2001). An adaptive finite element method for viscoplastic fluid flows in pipes. Computer Methods in Applied Mechanics and Engineering, 190(40-41):53915412.
[Staron et al., 2012] Staron, L., Lagrée, P.-Y., and Popinet, S. (2012). The granular silo as a continuum plastic flow: The hour-glass vs the clepsydra. Physics of Fluids, 24(10):103301.

[Staron et al., 2014] Staron, L., Lagrée, P. Y., and Popinet, S. (2014). Continuum simulation of the discharge of the granular silo. The European Physical Journal E, 37(1).

[Tasora, 2013] Tasora, A. (2013). Efficient simulation of contacts, friction and constraints using a modified spectral projected gradient method. In Poster Proceedings of WSCG 2013, pages 69-72.

[Wellmann and Wriggers, 2012] Wellmann, C. and Wriggers, P. (2012). A two-scale model of granular materials. Computer Methods in Applied Mechanics and Engineering, 205-208:46-58. 